Mational Sureau of Standards

\author{
AUG 211947
}

\title{
IONOSPHERC DATA ON FILE AT IRPL
}

This report is a convenient index of all ionospheric data on file in the offices of IRPL as of 1 Jamury 1946. Unless otherwise indicated, solid lines represent daily-hourly tabulations. Broken lines, in general, represent provisional data for which final tabulations are expected. However, when solid and broken lines appear in the same month, the broken lines represent provisionel data for which no daily-hourly tabulations are expected. The report can be kept up-to-date by extending the lines on the basis of the ionospheric data presented monthly in the IRPL-F series reports "Ionospheric Data".

Summaries of data on file at IRPL, with few exceptions, consist of average values through becember 1944, and of median values wherever that is possible from January 1945 to date. Where values avalable are averages only, that fact is indicated.

All distances in the mur and $M$ values are in kilometers unless otherwise noted.

The index has been arranged for breaking up into loose-leaf form or into separate sheets for convenient filing. Eight blank pages are included for indexing locel data end for keeping additional records.

The following list of stations is covered by the index.

Adak, Alaska
Alna Ata, U.S. 3. .
Athens, Greece
Bad Voeslau, Gemany
Baton Kouge, Houisiana
Bombay, India
Boston, Mas sachusetts
Brisbane, Australia
Bukhta Tikrava, U.S. S.R.
Burghead, Scotland
Cairo, Egypt
Campbell Island
Canberra, Australia
Capetown, Union of
S. Africa
Cape York. Australia
Christchurch, N.2.
Christmas Island

$$
\begin{array}{rr}
51.9^{\circ} \mathrm{N}, & 176.0^{\circ} \mathrm{H} \\
43.5^{\circ} \mathrm{N}, & 76.5^{\circ} \mathrm{E} \\
33.1^{\circ} \mathrm{N}, & 23.9^{\circ} \mathrm{E} \\
47.9^{\circ} \mathrm{N}, & 16.2^{\circ} \mathrm{E} \\
30.5^{\circ} \mathrm{N}, & 91.20^{\circ} \mathrm{N} \\
19.00 \mathrm{~N}, & 73.0^{\circ} \mathrm{E} \\
42.4^{\circ} \mathrm{N}, & 71.2^{\circ} \mathrm{N} \\
27.5^{\circ} \mathrm{S}, & 153.0^{\circ} \mathrm{E} \\
80.3^{\circ} \mathrm{N}, & 52.3^{\circ} \mathrm{E} \\
57.70 \mathrm{~N}, & 3.5^{\circ} \mathrm{N} \\
30.00 \mathrm{~N}, & 31.20^{\circ} \mathrm{E} \\
52.50^{\circ}, & 169.2^{\circ} \mathrm{E} \\
35.3^{\circ} \mathrm{S}, 149.00 \mathrm{E} \\
33.9^{\circ} \mathrm{S}, \quad 13.7^{\circ} \mathrm{E} \\
11.00 \mathrm{~S}, 142.4^{\circ} \mathrm{E} \\
43.5^{\circ} \mathrm{S}, 172.6^{\circ} \mathrm{E} \\
1.9^{\circ} \mathrm{N}, 157.3^{\circ} \mathrm{N}
\end{array}
$$




\begin{tabular}{|c|c|c|}
\hline Chungking, China & $29.4^{\circ} N_{0}$ & $106.8^{\circ} \mathrm{E}$ \\
\hline Churchil1. Canada & $58.80 \mathrm{~N}_{8}$ & $.20 \mathrm{Ni}$ \\
\hline Clyde, Baffin Island & $70.50 \mathrm{~N}$ & $68.6 \mathrm{~W}$ \\
\hline Colonbo, Ceylor & $6.60 N_{8}$ & $80.00 \mathrm{~F}$ \\
\hline Jea 1, New Jersoy & $74.00 \mathrm{~N}$ & $40.2 \%$ \\
\hline eIhi , India & $28.6 \mathrm{ON}_{8}$ & $77.2^{\circ} \mathrm{E}$ \\
\hline airbanks, Alaska & $64.9 \mathrm{ONY}_{\mathrm{S}}$ & $147.80 \mathrm{ch}$ \\
\hline roat Baddow, England & $51.7^{\circ} \mathrm{N}$ & $0.50 \mathrm{E}$ \\
\hline Guam Is land & $13.50 \mathrm{~N}$ & $144.8^{\circ} \Psi$ \\
\hline Iobart, Tosmanie & $43.0^{\circ} \mathrm{s}$ & $147.30 \mathrm{E}$ \\
\hline - llaridia, New Guinea & & $140.00 \mathrm{E}$ \\
\hline onshu, Japan & $O N_{0}$ & $135.0^{\circ} \mathrm{Fs}_{3}$ \\
\hline Huancayo, peru & $12.00 S_{9}$ & $75.30 \mathrm{~N}$ \\
\hline Ke rmadec Is lands & $29.2 \circ 5$ & $177.90 \mathrm{~W}$ \\
\hline 1. Gemany & $47.7^{\circ} \mathrm{N}$ & $11.4 \mathrm{OE}$ \\
\hline Kwa jalein A toll & $9.00 \mathrm{~N}_{0}$ & $168.0^{\circ} \mathrm{E}$ \\
\hline Leningrad, U.S.S.R. & ON, & $30.5^{\circ} \mathrm{E}$ \\
\hline Leyte. Philippine Is land s & & $125.00 \mathrm{E}$ \\
\hline Loshan. China & & $103.7^{\circ} \mathrm{E}$ \\
\hline Madras, India & 1 & $.2{ }^{\circ} \mathrm{E}$ \\
\hline Maui, Fawaii & $20.8^{\circ} \mathrm{N}$ & $156.5 \mathrm{~W}$ \\
\hline Voscows U.S.S.R。 & $55.8^{\circ} \mathrm{N}_{8}$ & $37.6^{\circ} \mathrm{s}$ \\
\hline 0s low, Norway & & $11.0^{\circ} \mathrm{S}$ \\
\hline wa. Canada & & 89 \\
\hline $\begin{array}{l}\text { Palau Islands } \\
\text { Poshawar. India }\end{array}$ & $\begin{array}{l}25.005 \\
34.0^{\circ} \mathrm{N}_{0}\end{array}$ & $\begin{array}{r}130 \\
71\end{array}$ \\
\hline Pitcairn Island & $25.0^{\circ} \mathrm{S}_{0}$ & $130.00 \mathrm{~W}$ \\
\hline Prine \& Rupert, Canada & $\mathrm{ON}_{\mathrm{N}}$ & $130.30 \mathrm{~W}$ \\
\hline ar ot onga I s land & & $159.69 \mathrm{Fi}$ \\
\hline Reyk javik, Iceland & $64.10 \mathrm{~N}$, & $21.70 \mathrm{~W}$ \\
\hline Johns, Newfound land & 47 & 52.79 \\
\hline Sakho Iin Is land & $N_{0}$ & $143.00 \mathrm{E}$ \\
\hline San Francisco, Californi & $37.4 \mathrm{ON}_{0}$ & $122.29 w$ \\
\hline San Juan, Puerto Rico & 1 & $66.10 \mathrm{~W}$ \\
\hline Slough, England & & $0.69 \mathrm{wi}$ \\
\hline Slutsk。UoS。S.ti。 & $59.7^{\circ} \mathrm{N}$ & $30.5 \mathrm{OE}$ \\
\hline Snainton, England & $54.20 \mathrm{~N}$ & $0.60 \mathrm{O}$ \\
\hline Spitsbergen, Norway & $78.2^{\circ} \mathrm{No}$ & $15.6^{\circ} \mathrm{E}$ \\
\hline Sveralovsk, U.SoS.R. & & $61.10 \mathrm{E}$ \\
\hline Syracuse, Sicily & & $15.2^{\circ} \mathrm{E}$ \\
\hline Tokjo, Japan & $35.6 \mathrm{O}_{\mathrm{N}}$ & $139.6^{\circ} \mathrm{E}$ \\
\hline Tomsk, U.S.S.R。 & $56.49 \mathrm{~N}$ & $85 . \mathrm{C}^{\circ} \mathrm{E}$ \\
\hline Trinidad。Britow。Indies & $10.60 \mathrm{~N}$ & $61.29 \mathrm{H}$ \\
\hline Tromsos Norway & $69.6^{\circ} \mathrm{N}$ & $19.00 \mathrm{E}$ \\
\hline Victoria Beach, Canada & $50.8^{\circ} \mathrm{N}$ & 96.59 \\
\hline ashington, $D \circ C$ 。 & $39.00 \mathrm{~N}$ & 77.5 이방 \\
\hline roo, Wo Austral & & $115.90 \mathrm{E}$ \\
\hline Tuchang, China & $30.6^{\circ} \mathrm{N}_{2}$ & $114.4^{\circ}$ \\
\hline
\end{tabular}




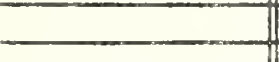

I

Absorption 

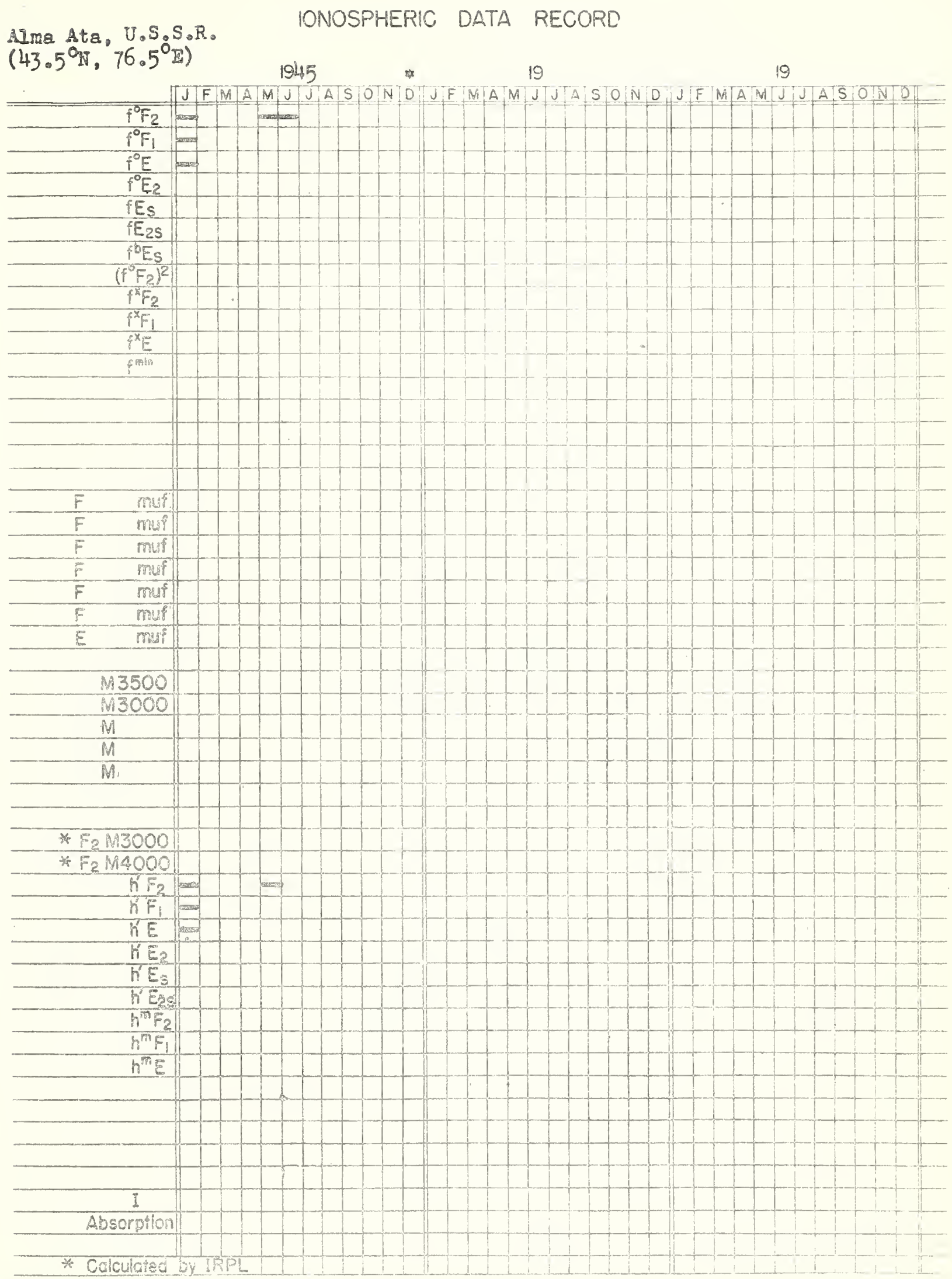

wonshy averages by racilo 



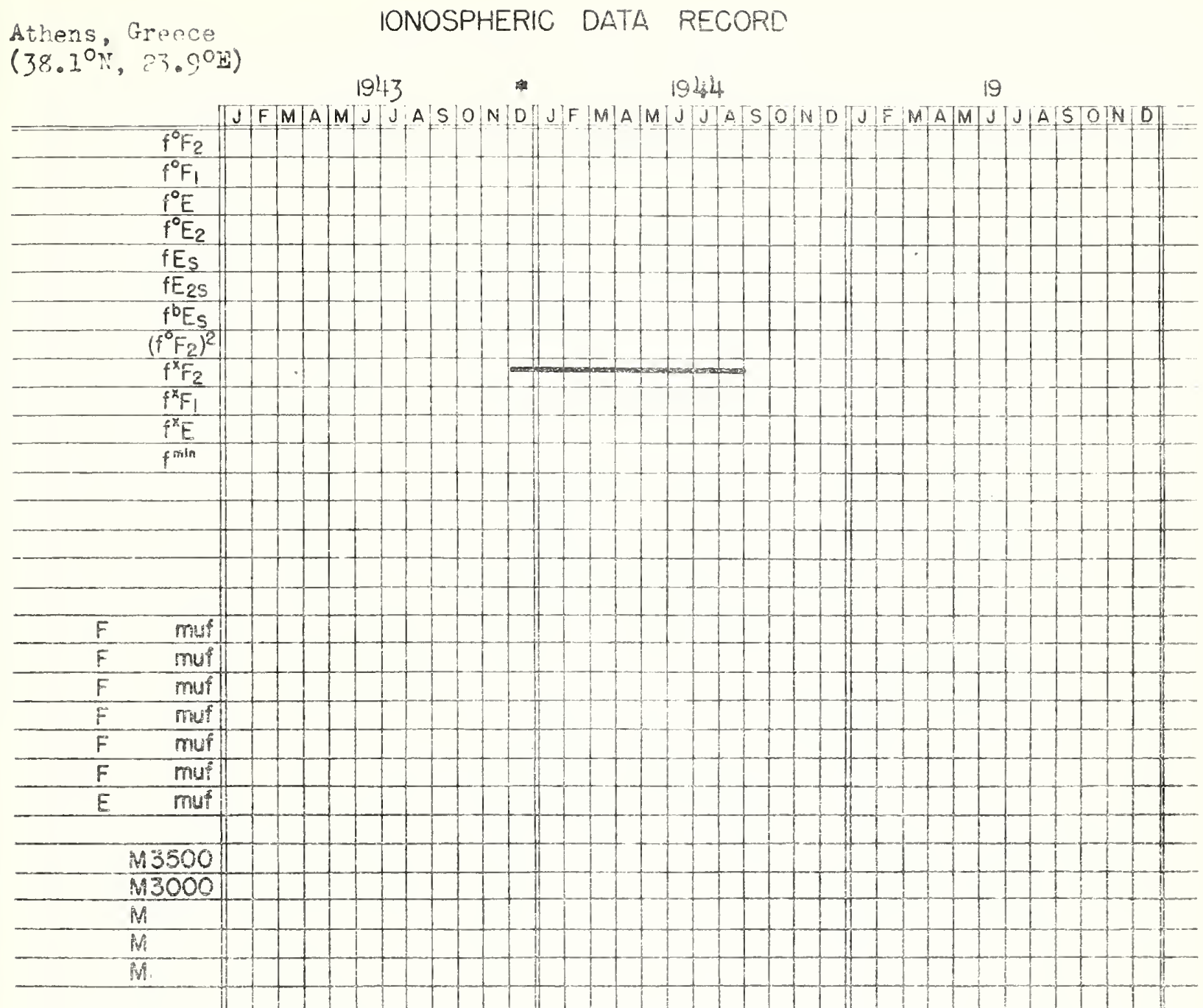

* FaM3000

* Fenta00 hFa

his

h

กิ

"re

$h^{\text {mon }}$

$h^{\text {min }} F_{1}$

$h^{m}$

Aluserghion

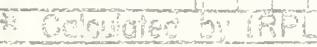

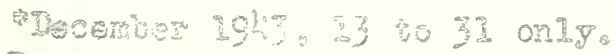

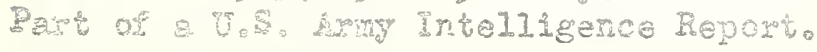



Raton Rorge, Lorisiana

\section{IONOSPHERIC DATA RECORD}

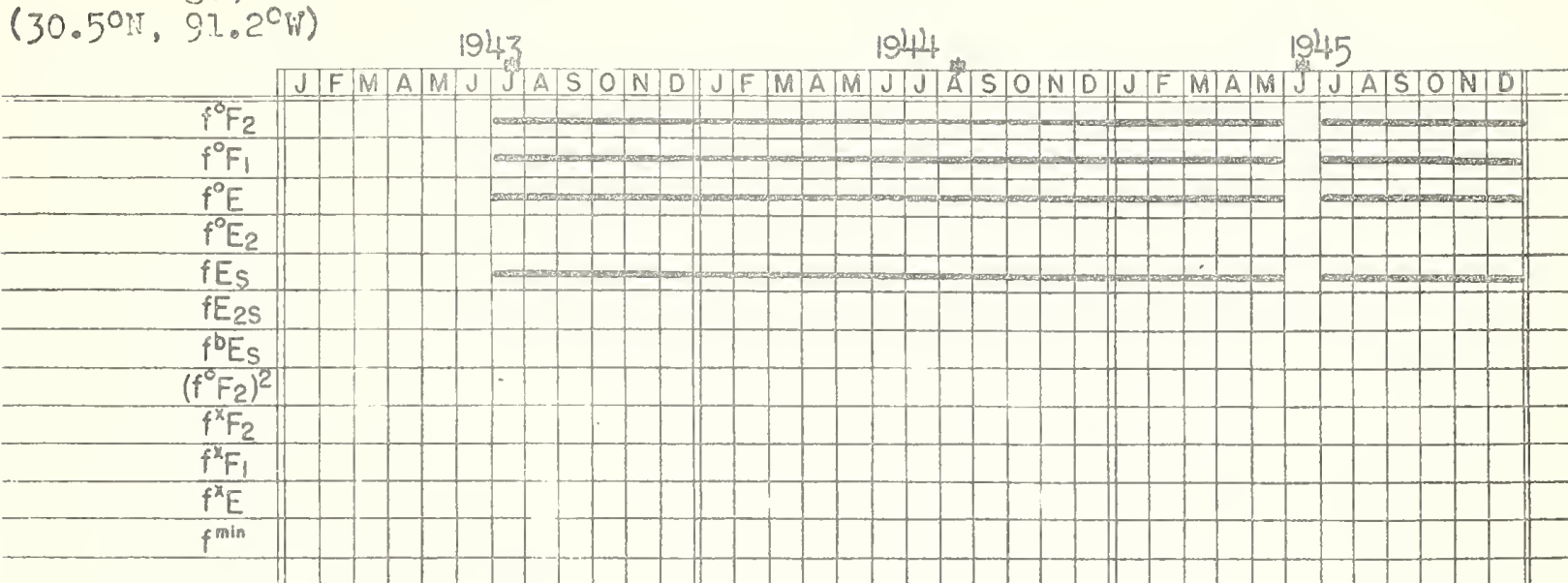

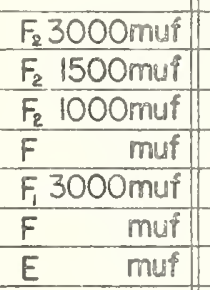

$M 3500$

E2M3000

$\frac{M}{M}$

$\mathrm{M}$

烈1 193000

* $F_{2} M 3000$

* $\mathrm{F}_{2} \mathrm{M} 4000$

$h^{\prime} \mathrm{F}_{2}$

$h^{\prime} F_{1}$

hE

$h^{\prime} E_{2}$

$h^{\prime} E_{S}$

$h^{\prime} E_{25}$

$h^{m} F_{2}$

$h^{m} F_{1}$

$h^{m} E$$$
\text { (n) }
$$

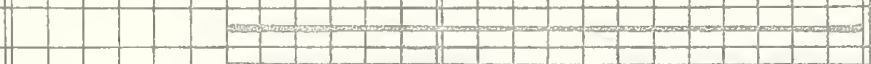

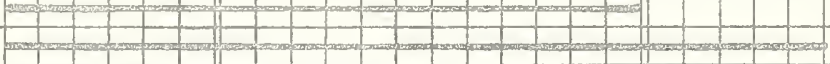

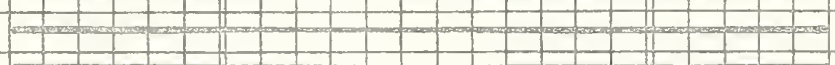

(1)

$\frac{-10}{2}$

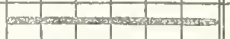

and

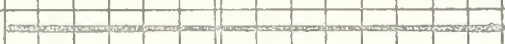

anm

- 1 - 


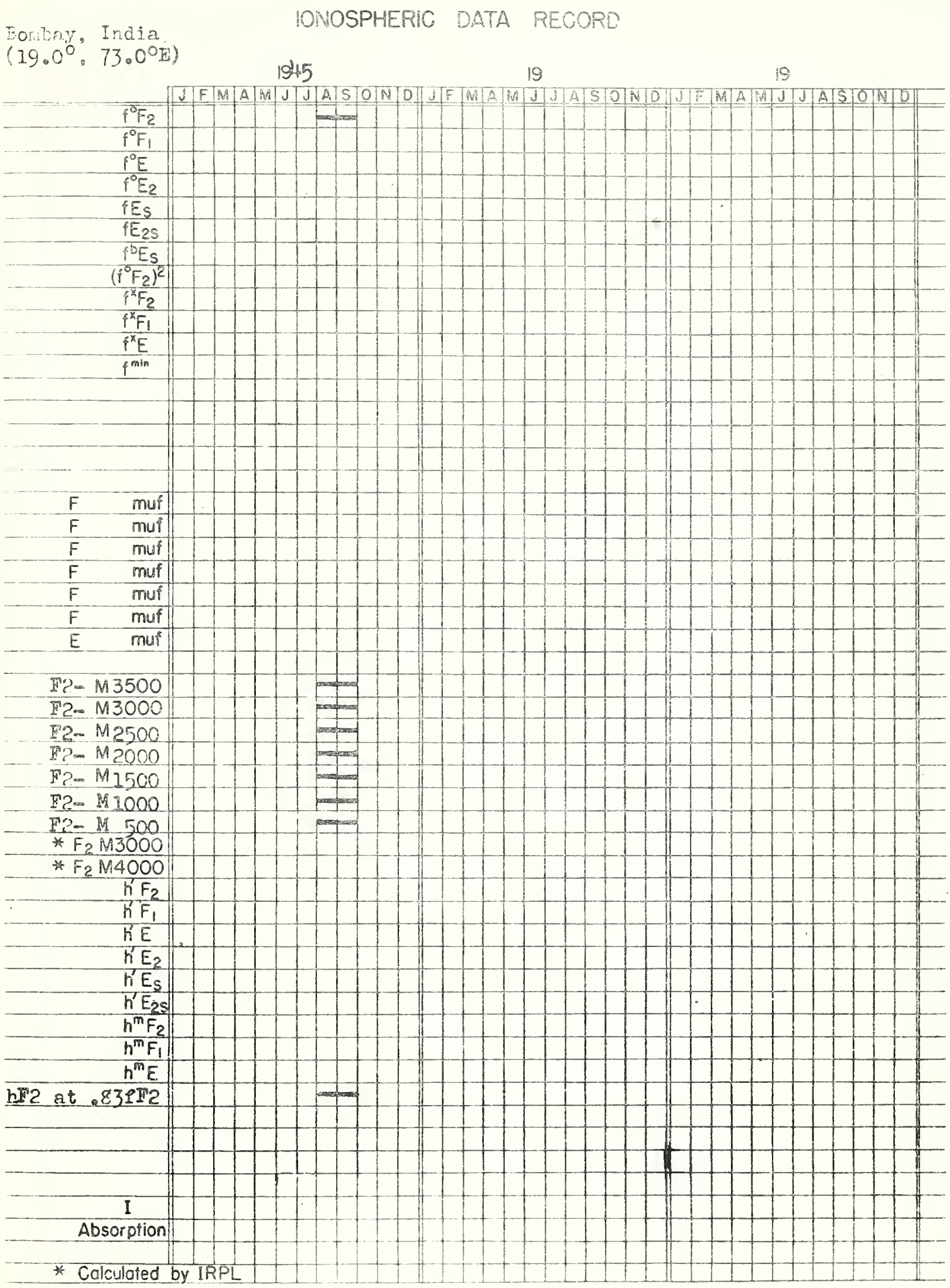

Monthly averames nnly. 



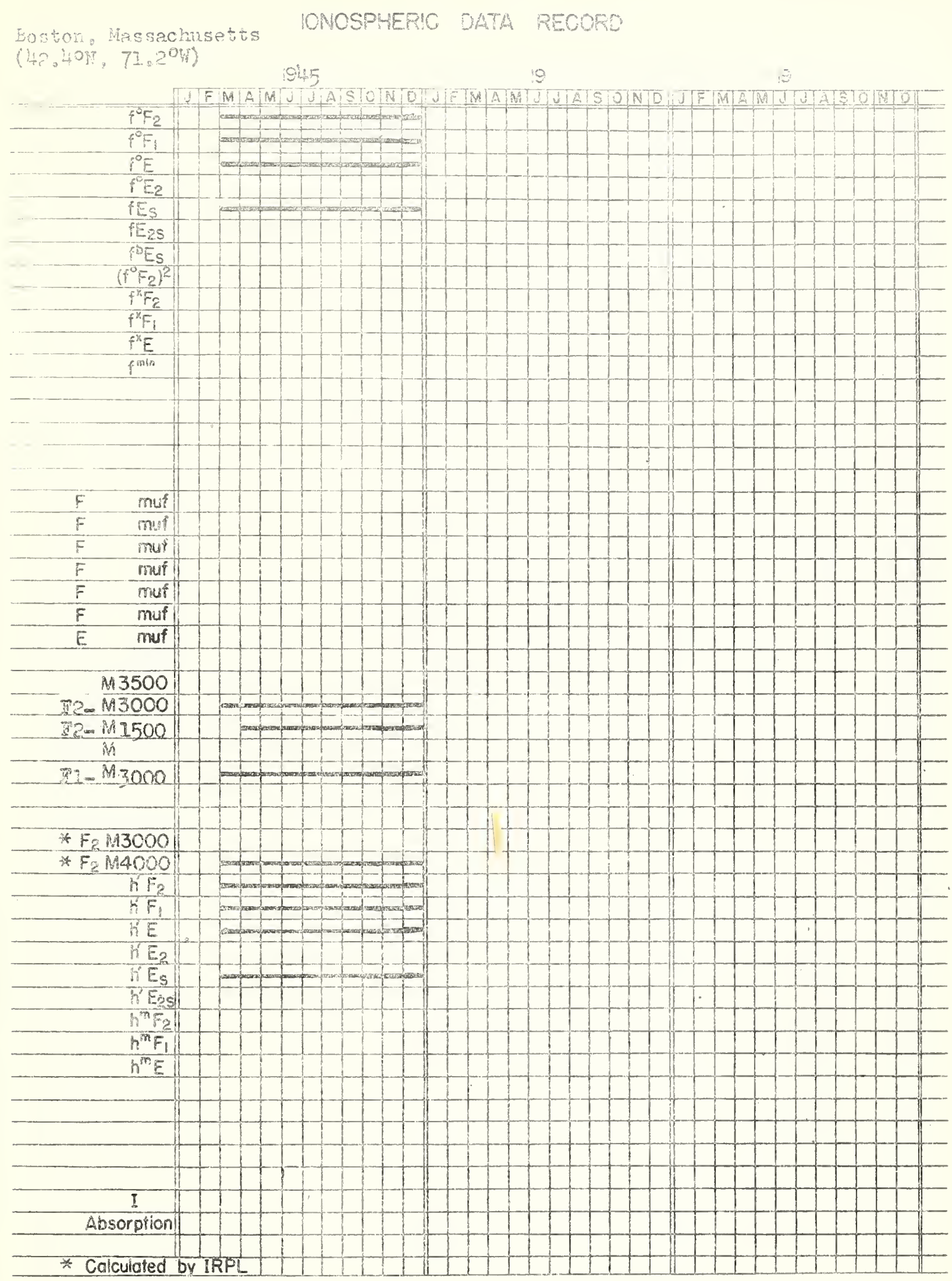





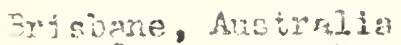

$(2, .5 \mathrm{~S}, 15 ; .00 \mathrm{E})$

1943

1944

1945

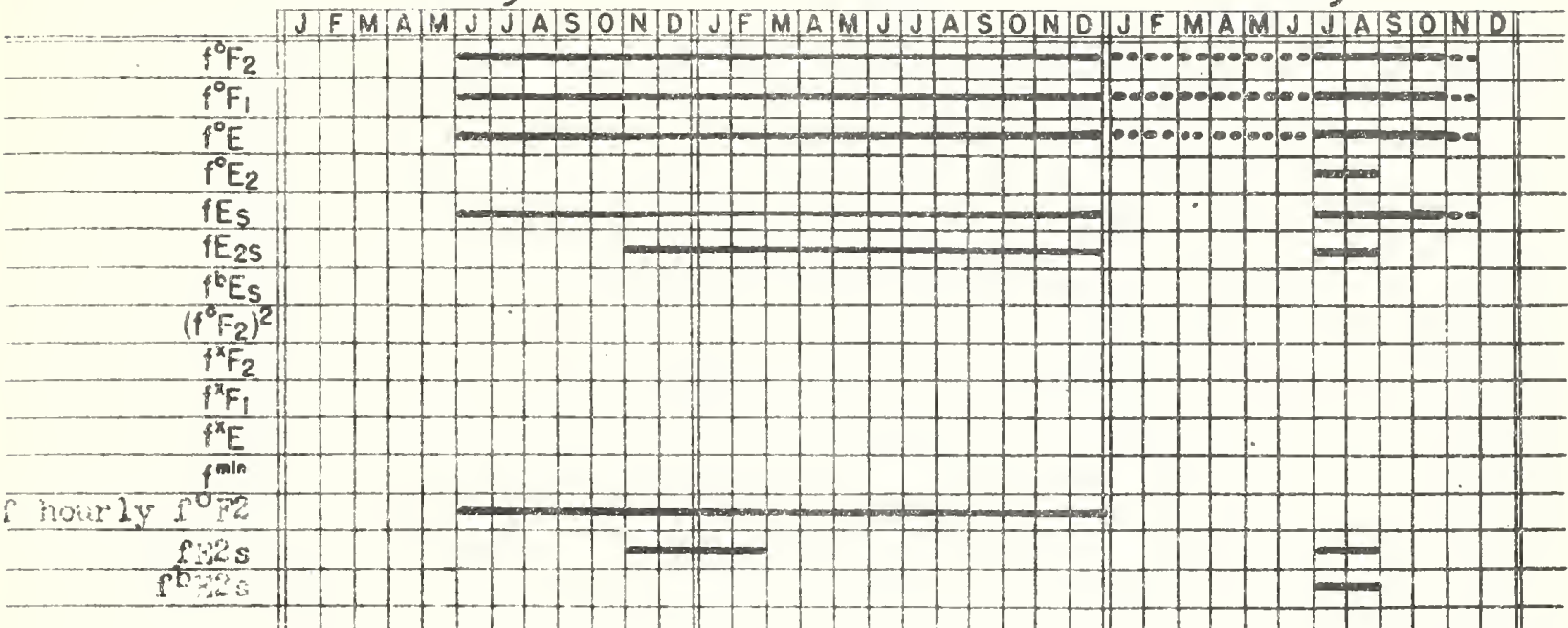

min 10 horly fore

$$
\text { riss }
$$

\begin{tabular}{rr}
$F_{2} 3000$ muf \\
\hline$F$ & muf \\
\hline$F$ & muf \\
\hline$F$ & muf \\
\hline$F$ & muf \\
\hline$F$ & muf \\
\hline$E$ & muf
\end{tabular}

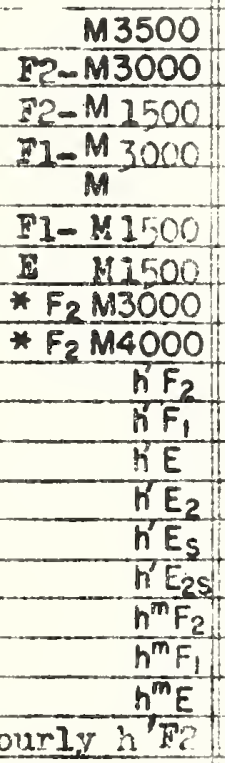

half hourly $\mathrm{h}$. Fo
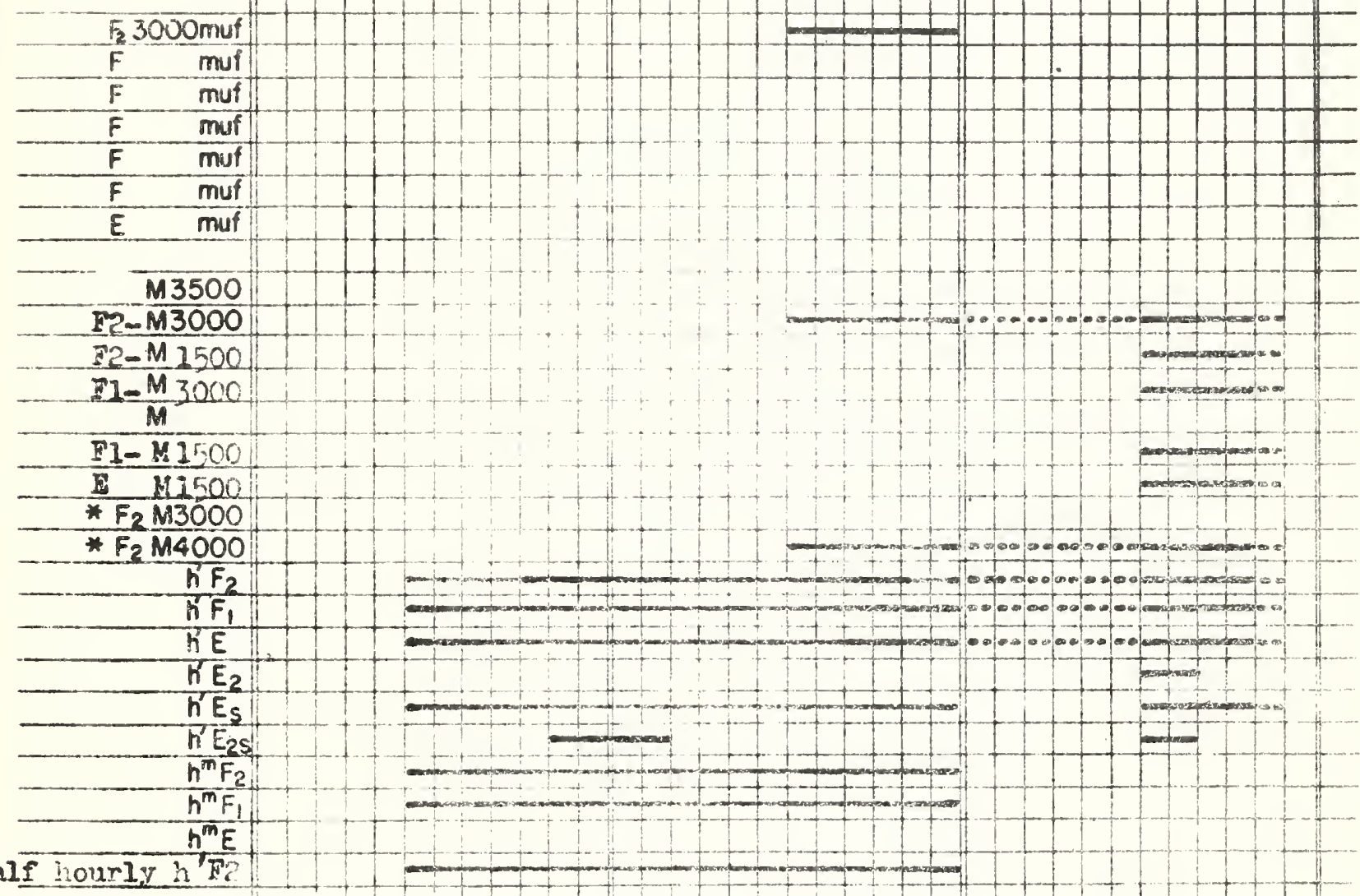

Absorption

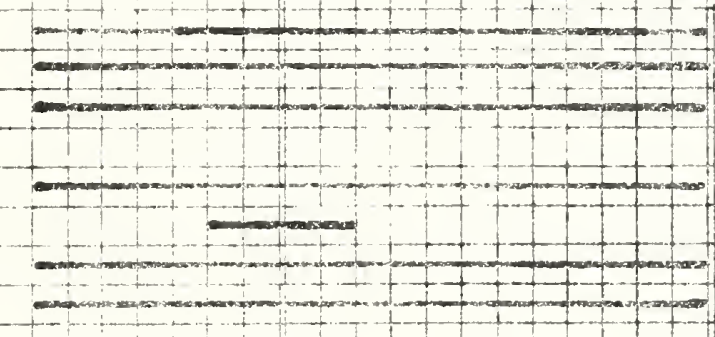

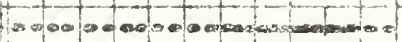

romento

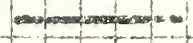

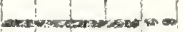

mand on

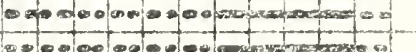
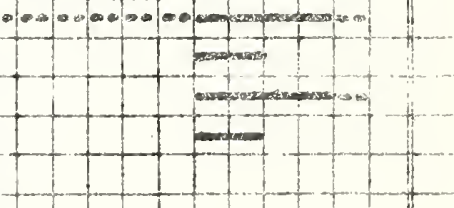

Whte Tikhera, U.S.S.R. IONOSPHERIC DATA RECORD

$\left(x, \cdot z^{0}, 52.80 \mathrm{E}\right)$

1944

1945

19

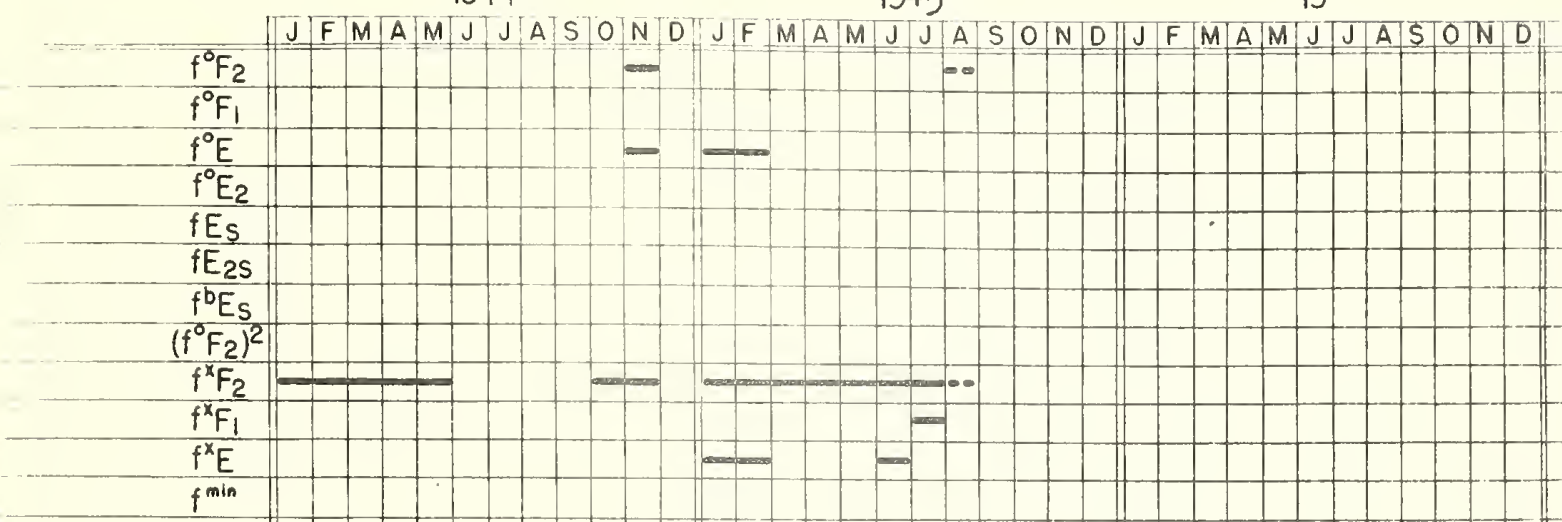

\begin{tabular}{rr|}
\hline$F$ & muf \\
\hline$F$ & muf \\
\hline$F$ & muf \\
\hline$F$ & muf \\
\hline$F$ & muf \\
\hline$F$ & muf \\
\hline$E$ & muf
\end{tabular}

M3500

M3000

$M$

$\dot{M}$

$+7$

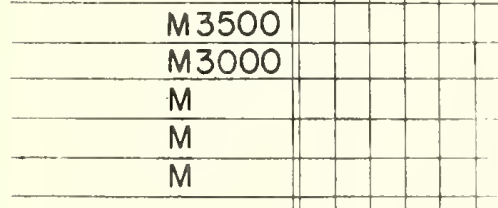

* $\mathrm{F}_{2} \mathrm{M} 3000$

* $\mathrm{F}_{2} \mathrm{M} 4000$

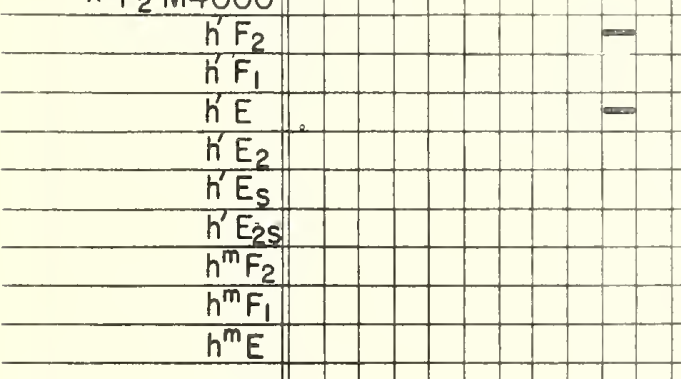

\begin{tabular}{l|}
$h^{\prime} F_{2}$ \\
\hline$h^{\prime} F_{1}$ \\
\hline$h^{\prime} E$ \\
\hline$h^{\prime} E_{2}$ \\
$h^{\prime} E_{5}$ \\
$h^{\prime} E_{2 s}$ \\
$h^{m} F_{2}$ \\
$h^{m} F_{1}$ \\
\hline$h^{m} E$
\end{tabular}

Absorption

* Calculated by IRPL

Monthly averages only. 

Bukhta Tikhaya, U.S.S.R. IONOSPHERIC DATA RECORD
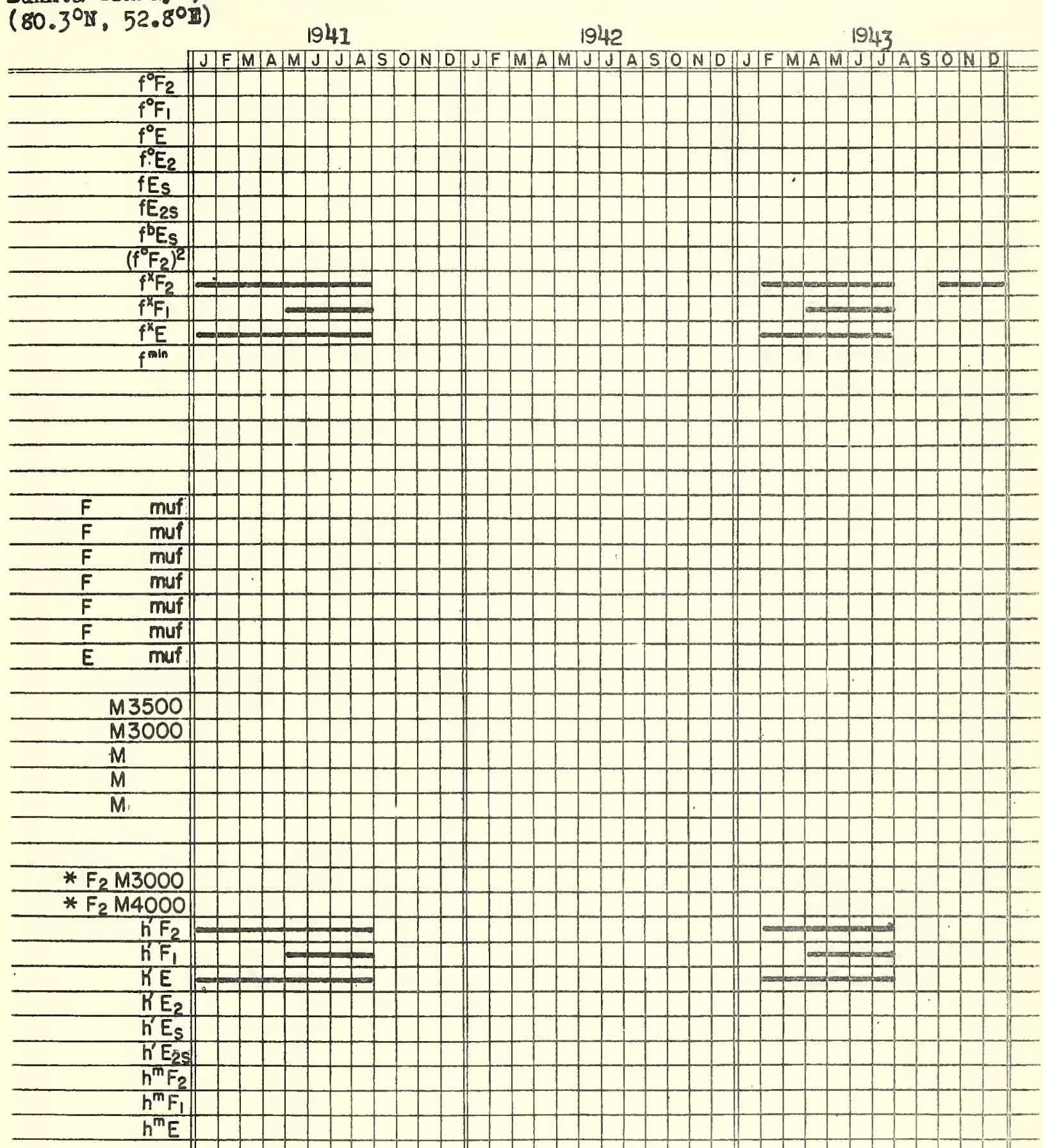

Absorption

* Calculated by IRPL 

Bukhta Tikhaya, U.S.S.R.

IONOSPHERIC DATA RECORD

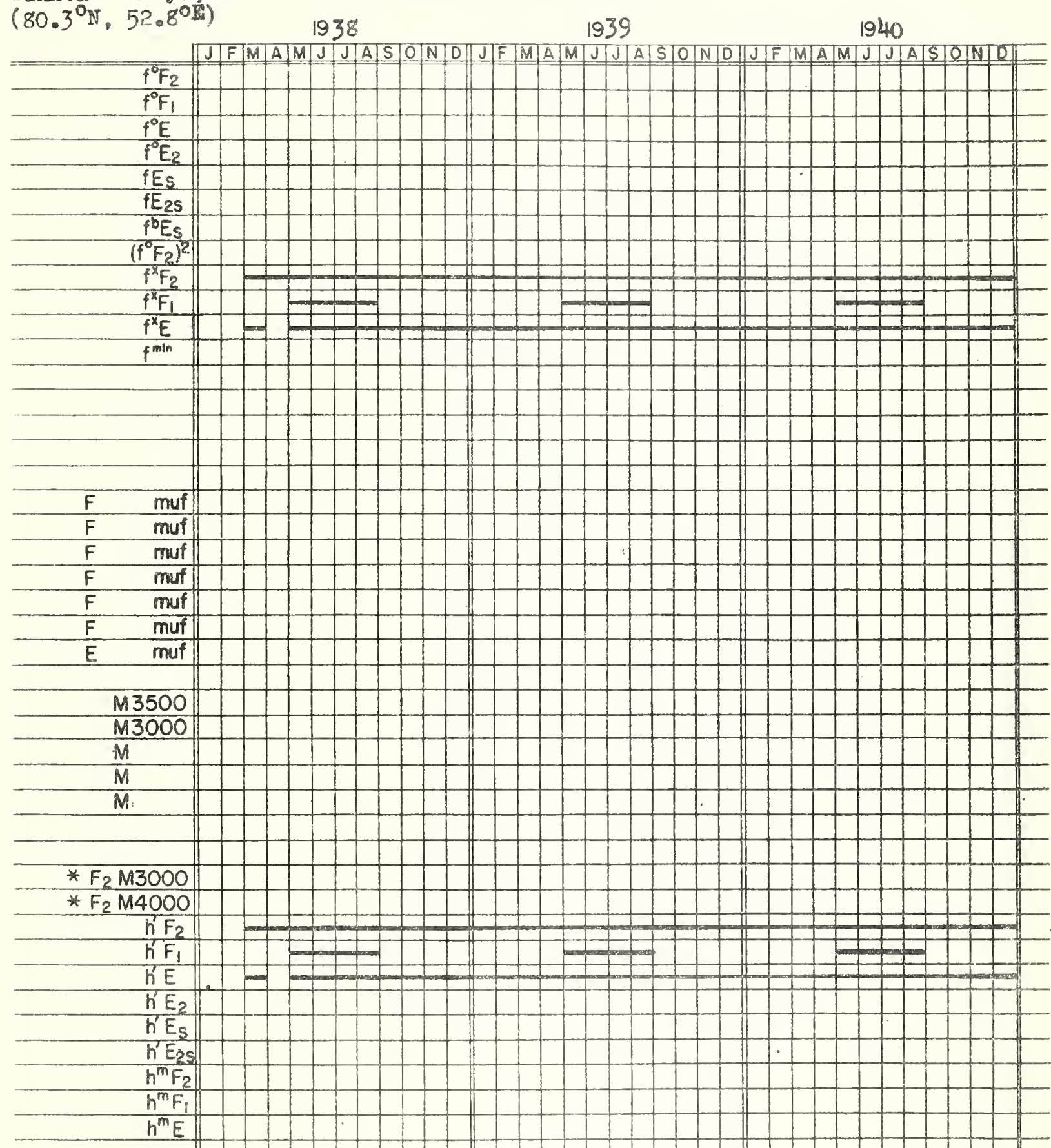



IONOSPHERIC DATA RECORD

$\left(57.79 \mathrm{~N}, 3.5^{\circ} \mathrm{W}\right)$

1945

19

19

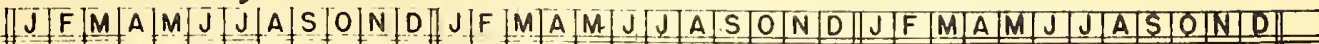

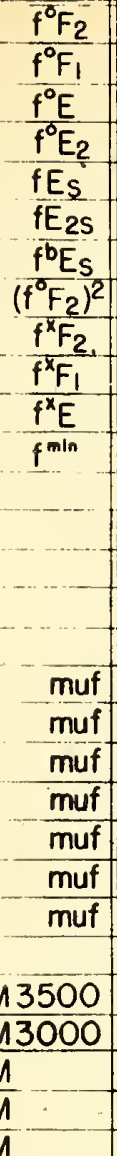

\begin{tabular}{ll}
$F$ & muf \\
$F$ & muf \\
\hline$\frac{F}{F}$ & $\frac{\text { muf }}{\text { muf }}$ \\
\hline$F$ & $\frac{\text { muf }}{E}$ \\
\hline$\frac{M 3500}{\text { muf }}$ \\
$\frac{M 3000}{M}$ \\
$\frac{M}{M}$
\end{tabular}

* $F_{2} M 3000$

* $\mathrm{F}_{2} \mathrm{M} 4000$

$h^{\prime} F_{2}$

$h^{\prime} F_{1}$

$h^{\prime} E$

$h^{\prime} E_{2}$

$h^{\prime} E_{S}$

$h^{m} F_{2}$

$h^{m} F_{1}$

$h^{m} E$

$-$ 
. 
Burghead, Scotiand

$\left(57.7^{\circ} \mathrm{N}, 3.5^{\circ} \mathrm{H}\right)$
IONOSPHERIC DATA FECORO

1942

194

1944

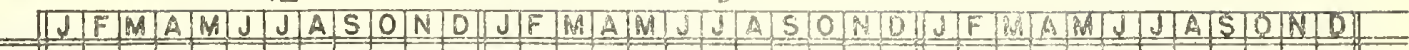

$f^{\circ} F_{1}$

$f^{\circ} \mathrm{E}$

fEs

$\mathrm{fE}_{2 \mathrm{~S}}$

$\mathrm{Fb}_{\mathrm{b}}$

$\frac{\left(f^{8} \mathrm{~F}_{2}\right)^{2}}{f^{\times} \mathrm{F}^{2}}$

$f^{2} F_{i}$

$f^{\mathrm{x}} E$

fom

\begin{tabular}{|c|c|}
\hline$F$ & $\bar{m}$ \\
\hline$F$ & \\
\hline$F$ & \\
\hline$F$ & \\
\hline$F$ & \\
\hline$F$ & \\
\hline$E$ & \\
\hline
\end{tabular}

M 3500

A 3000

M

的

M

* F. M3000

* Fon $N_{000}$

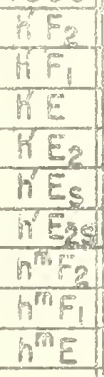

E

KEZ

Es

inges

$\frac{m}{6 f_{1}}$

का 

IONOSPHERIC DATA RECORO

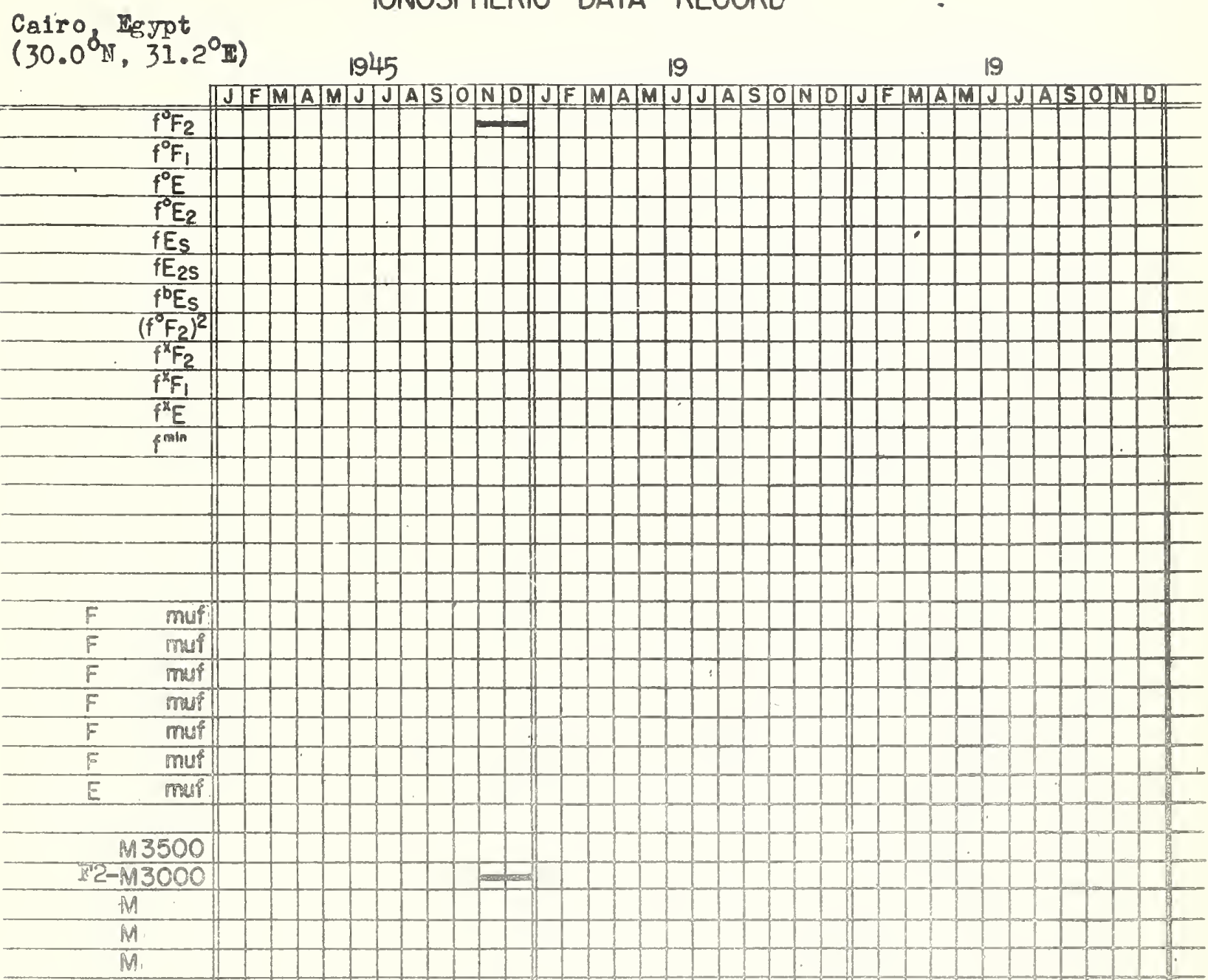

\begin{tabular}{r}
$* F_{2} M 3000$ \\
$* F_{2} M 4000$ \\
\hline$h^{\prime} F_{2}$ \\
$h^{\prime} F_{1}$ \\
\hline$h^{2} E$ \\
\hline$h^{\prime} E_{2}$ \\
$h^{\prime} E_{s}$ \\
$h^{\prime} E_{25}$ \\
$h^{m} F_{2}$ \\
$\frac{h^{m} F_{1}}{h^{m} E}$
\end{tabular}

Absorption

* Calculated by IRPL 

Carpbel1 I.

(52.505, 169.0 $0^{\circ}$.
IONOSPHERIC DATA RECORD

1944t

1945

19

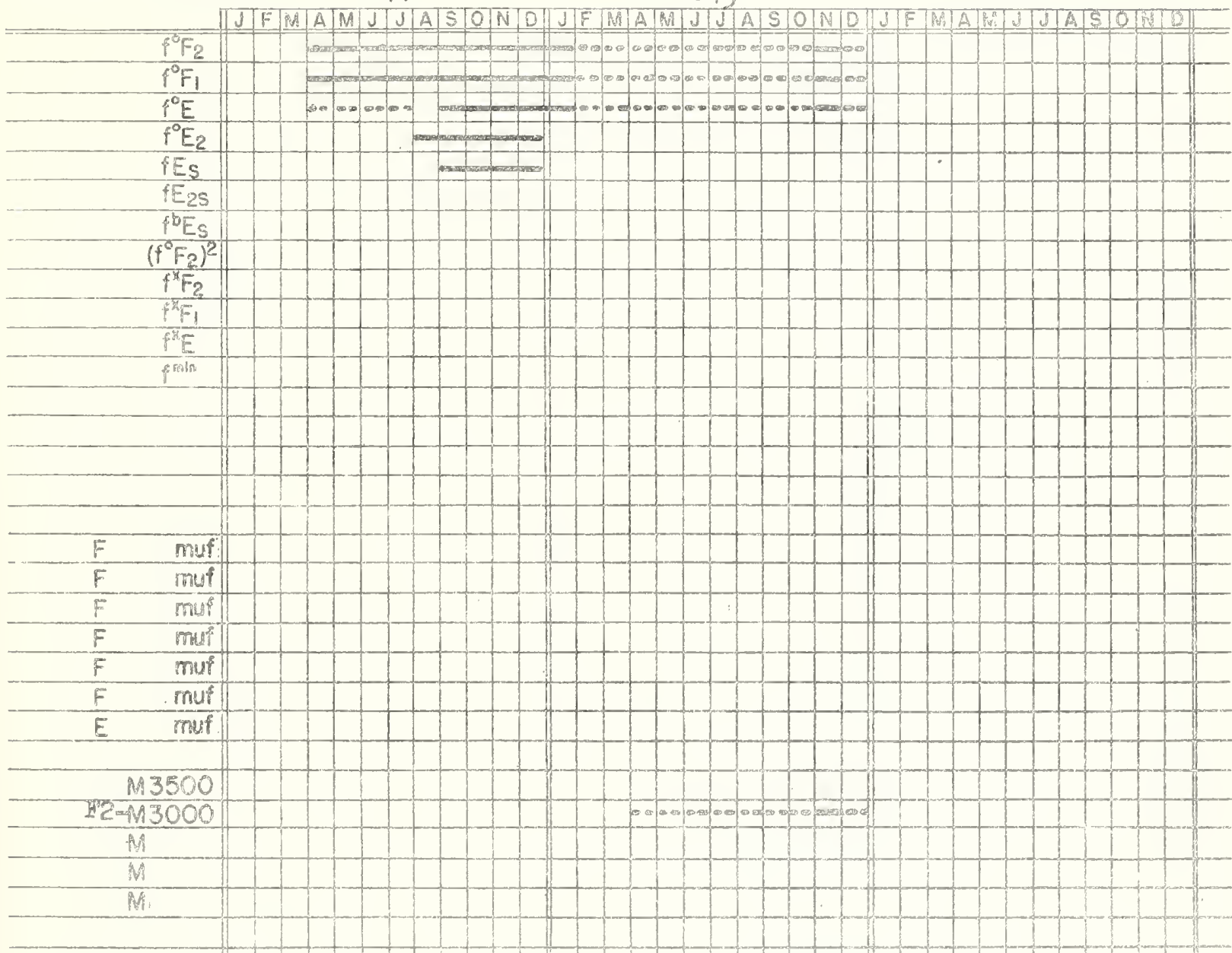

* FoM $M 000$

* F M4000

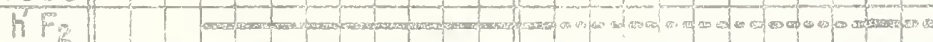

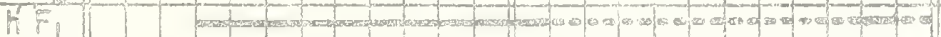

KE E

hE.

h.

$h^{\top} E_{2}$

$h^{\text {nin }} F_{3}$

$h^{m i n}$

$h^{m}$

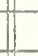

\begin{abstract}
1
\end{abstract}
$+$

Absorpfion

* Calculated by IRPL 
Canberra, Australia

$\left(35.3^{\circ} \mathrm{S}, 149.0^{\circ} \mathrm{E}\right)$
IONOSPHERIC DATA RECORD

1944

19

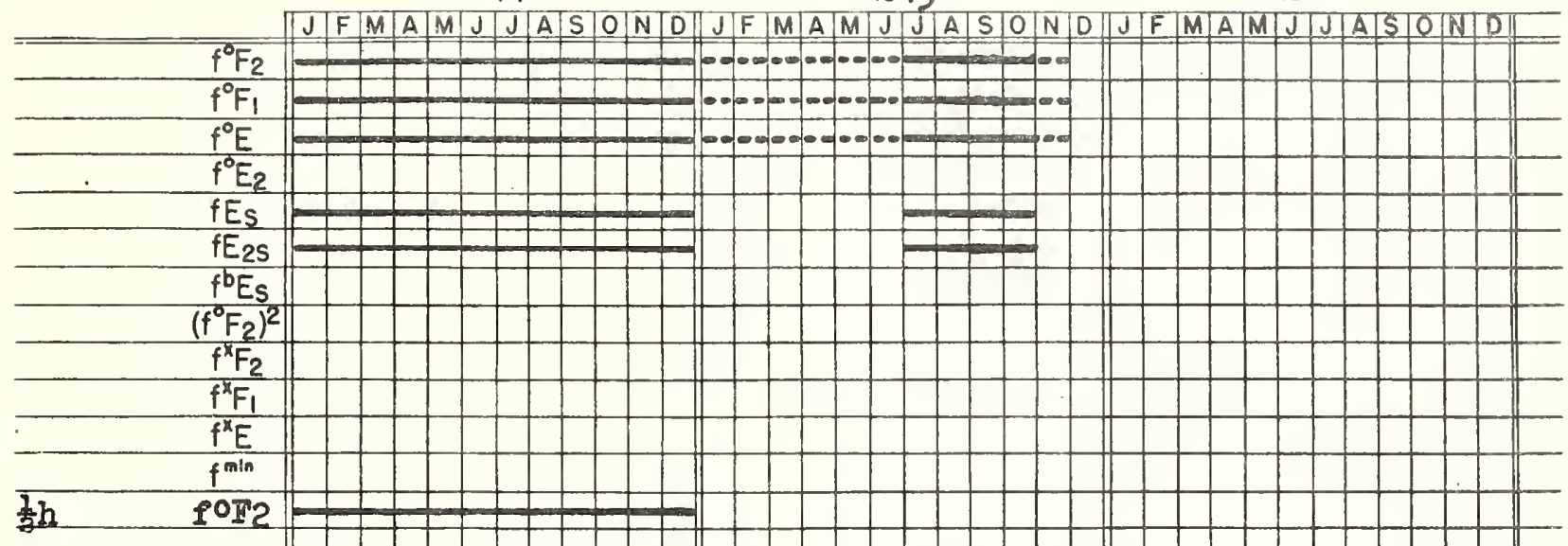

M3500

F2- $M 3000$

F2- M 1500 $M$

FI- M 3000

FI- 1500

$\quad 11500$

* $F_{2} M 3000$

* $\mathrm{F}_{2} \mathrm{M} 4000$

$\mathrm{h}^{\prime} \mathrm{F}_{2}$

$h^{\prime} F_{1}$

$h^{\prime} \mathrm{Y}+1-00000-000$

$h^{\prime} E_{2}$

$h^{\prime} E_{S}$

$h^{\prime} E_{2 s}$

$h^{m} F_{2}$

$h^{m} F_{1}$

I hour I g h/s2

1



Canberra, Asuctralia

IONOSPHERIC DATA RECORD

$\left(35.3^{\circ} \mathrm{S}, 149.0^{\circ}\right)$

1949

1942

1943

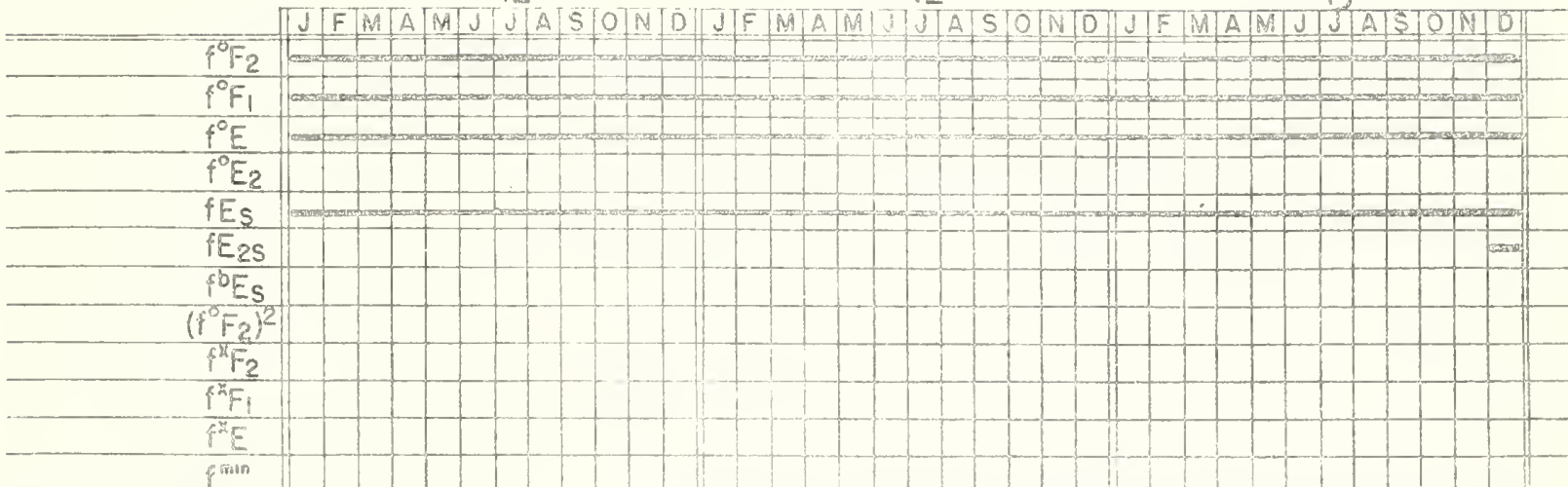

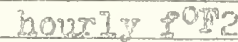

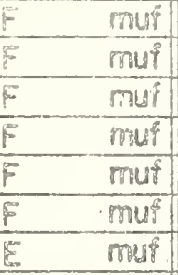

M 3500

M 3000

$M$

MA

M

法 $F_{2} M 13000$

it $F_{2} M 4000$

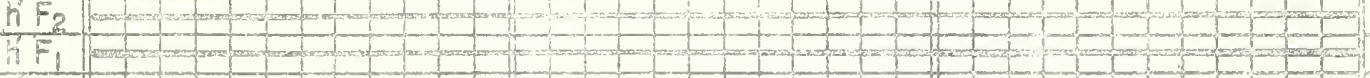

ME

$\mathrm{Re}_{2}$

$7 \mathrm{E}_{0}$ -

$h^{7} e_{\text {s. }}$

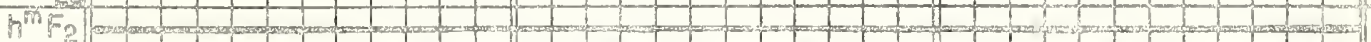

$h^{i} F_{1} 172$ t m m

tis.

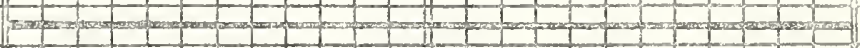

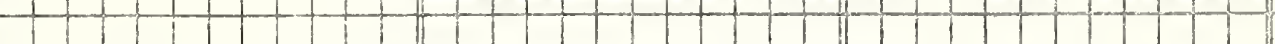

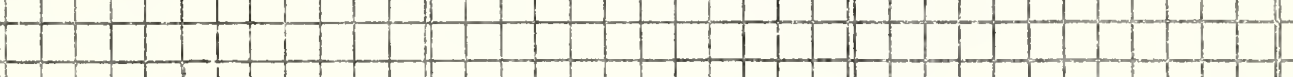
Absorption

* Colculoted by IRP 
Capetow, Union of $S$. APTICESPHERIC DATA RECORD

$\left(33.905,18.7^{\circ} \mathrm{E}\right)$

1944

$1945 \quad 19$

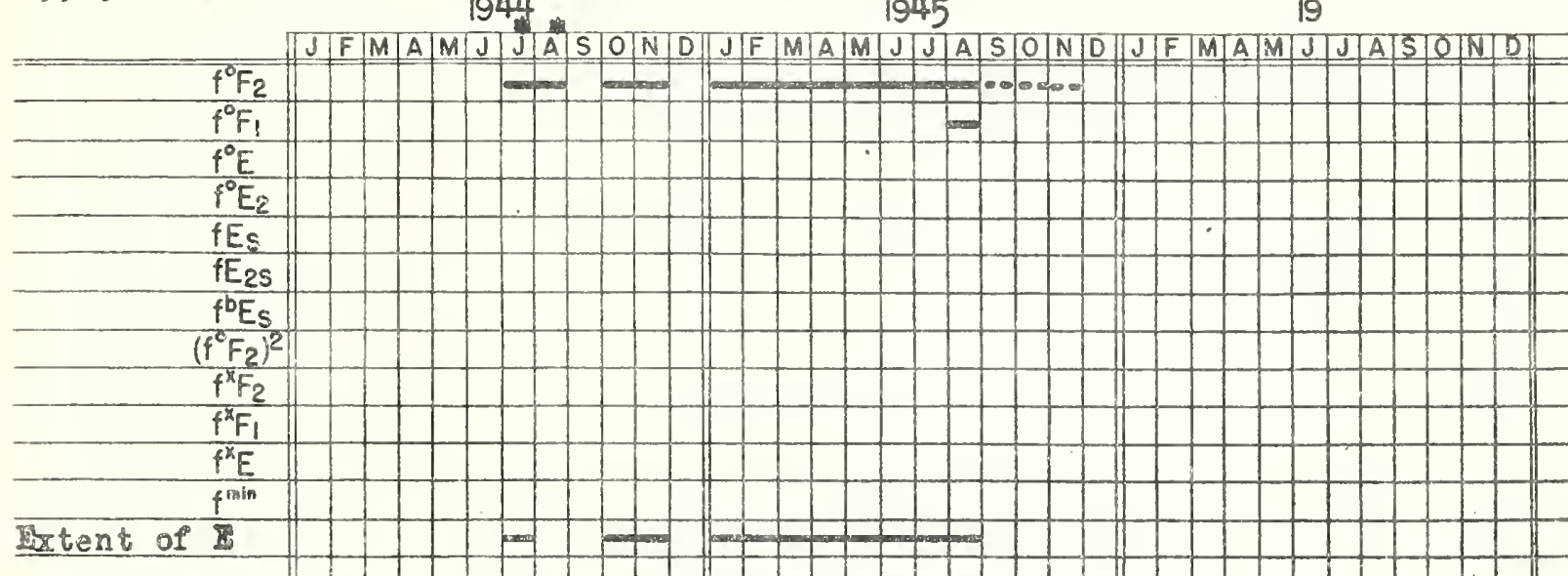

\begin{tabular}{cc|}
\hline$F$ & muf \\
\hline$F$ & muf \\
\hline$F$ & muf \\
\hline$F$ & muf \\
\hline$F$ & muf \\
\hline$F$ & muf \\
\hline$E$ & muf \\
\hline
\end{tabular}

$\frac{M 3500}{M 3000} \frac{M}{M}$

* $\mathrm{F}_{2} \mathrm{M} 3000$

* $\mathrm{F}_{2} \mathrm{M4000}$

$h^{\prime} F_{2}$

h'F

KE

$T^{n} E_{2}$

$h^{\prime} E_{S}$

$h^{\prime} E_{5}$

$h^{\text {m } F}$. $h^{m} \varepsilon$

Absorption

* Calculated by IRPL

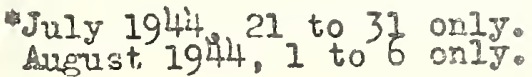


Sape York, Australia

(11.0 $\left.0^{\circ}, 142.40 \mathrm{~F}\right)$

\section{IONOSPHERIC DATA RECORD}

1944

1945

19

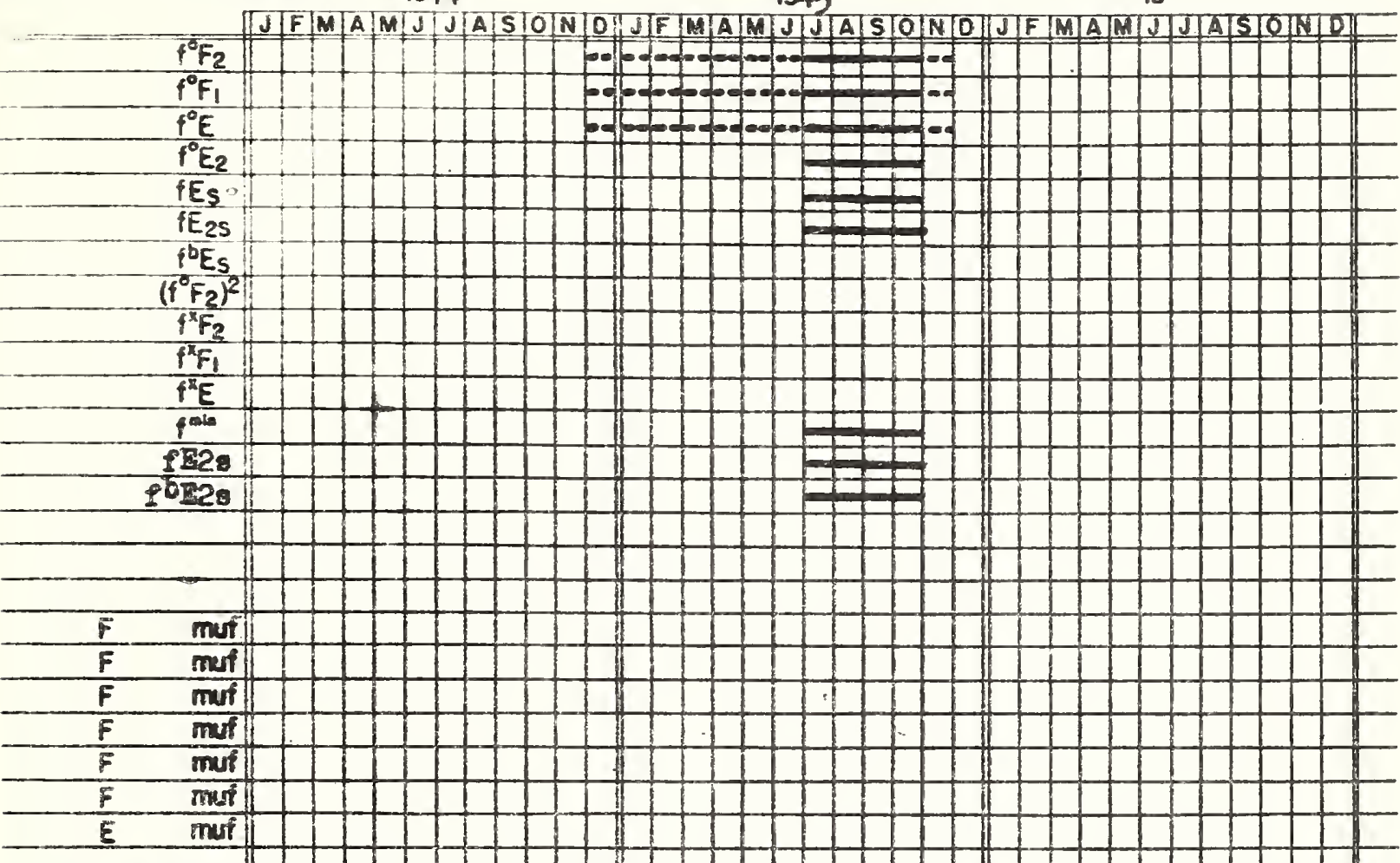

$M 3500$

P2 M3000

2201500

II 13000

II M1500

T $\quad$ 1500

* $F_{2} M_{3000}$

* Fr M000

$\mathrm{TF}_{2}$

${ }_{i} F_{i}$

त

E E

त $\varepsilon_{5}$

RE

$n^{n} F_{3}$

$\frac{h^{m} F_{1}}{h^{m} E}$

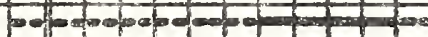

pand

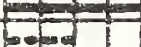

$-1$

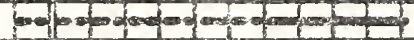

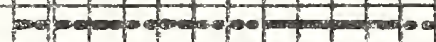

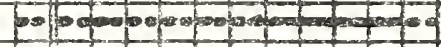

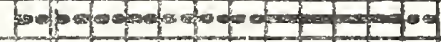

and

materas

$\rightarrow+1$ 

Christchureh, N.2.

IONOSPHERIC DATA RECORD

(43.5 ${ }^{\circ}, 172.6^{\circ}$ II)

19

19

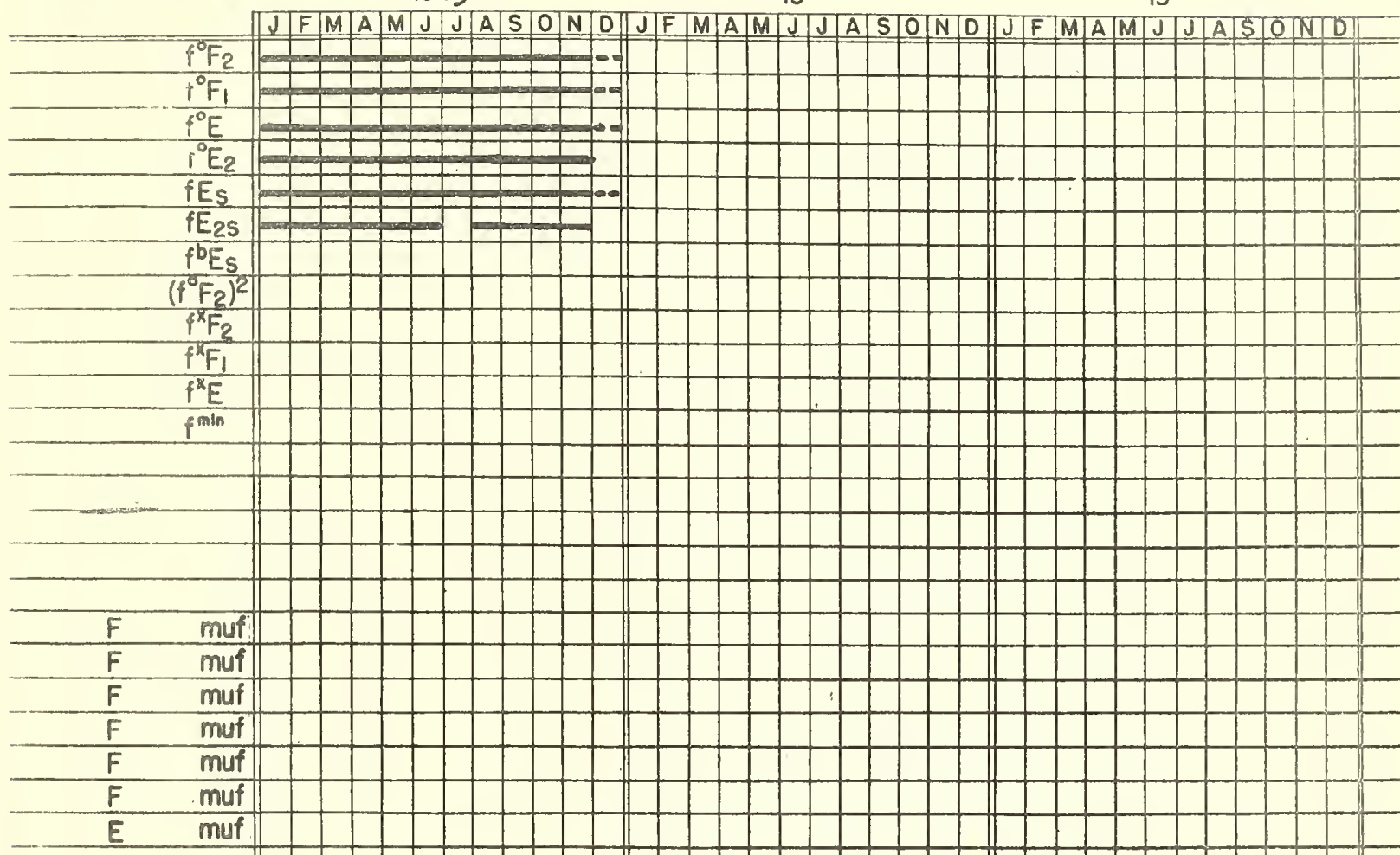

$\frac{M 3500}{M 3000}$

* $\mathrm{F}_{2} \mathrm{M} 3000$

* Fr M4000

$h^{\prime} F_{2}+1-0$

$h^{\prime} F_{1}+1-0$

KE

$h^{\prime} E_{2}+1$

$h^{\prime} E_{S}=11$

$h^{\prime} E_{2 s}$

$h^{\text {m }} F_{2}$

$h^{\text {in } F_{1}}$

$h^{\text {min } E}$

Absorpition

* Calculated by IRPL 

christerrei, $\%$ z. IONOSPHERIC DATA RECORD

$\left(45.5^{\circ} S, 77,50\right)$
19424
1943

194.4 .4

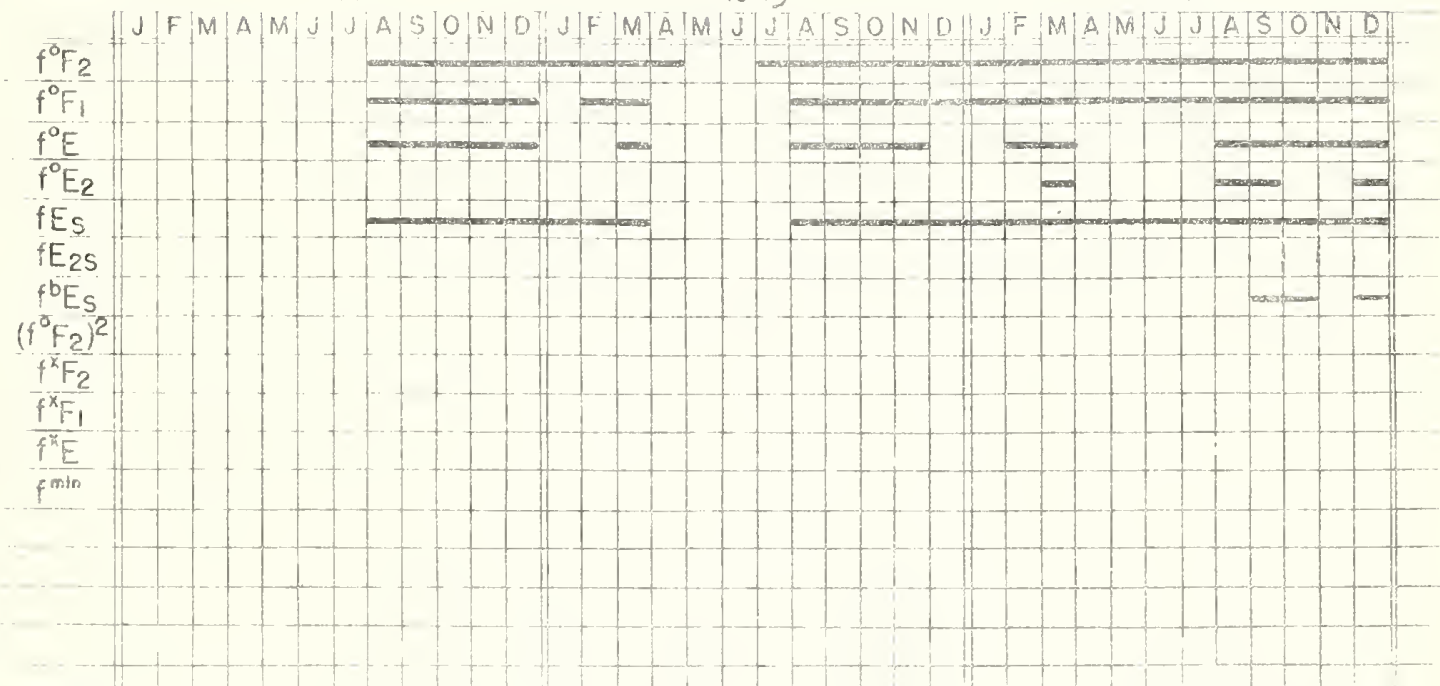

$$
\begin{array}{|c|c|}
\frac{F}{F}-\frac{m u f}{m} & \frac{m u f}{m u f} \\
\hline \frac{F}{F}-\frac{m u f}{m u f} & \frac{F}{C}-\frac{m u f}{\text { nuf }} \\
\hline-\frac{M 3500}{M 3000} & \frac{M}{M} \\
\hline \frac{M}{M}
\end{array}
$$

$F_{2} M 3000$

* $\mathrm{F}_{2} \mathrm{i} 14000$

$\uparrow F_{2}$

TiF

${ }^{\prime} E_{2}$

$n^{\prime} E_{S}$

$h^{\prime} E_{2 s}$

$\frac{h^{m} F_{2}}{h^{m} F_{1}}$

$h^{m} E$

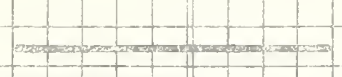

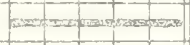

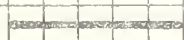

$\frac{1}{1}$ 

Christchurch, N. 2.

IONOSPHERIC DATA RECORD

$\left(43.5^{\circ} \mathrm{S}, 172.6^{\circ} \mathrm{E}\right)$

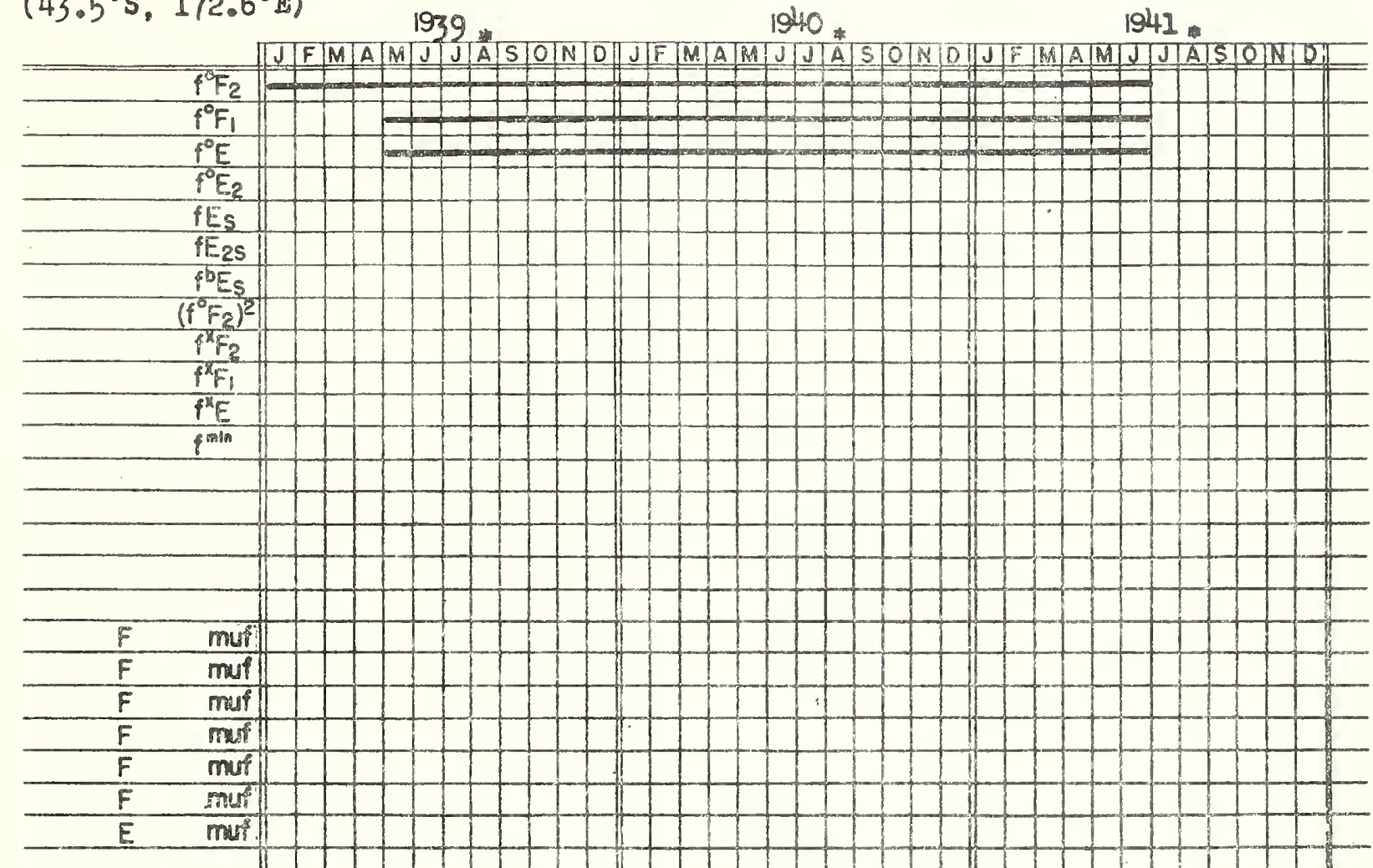

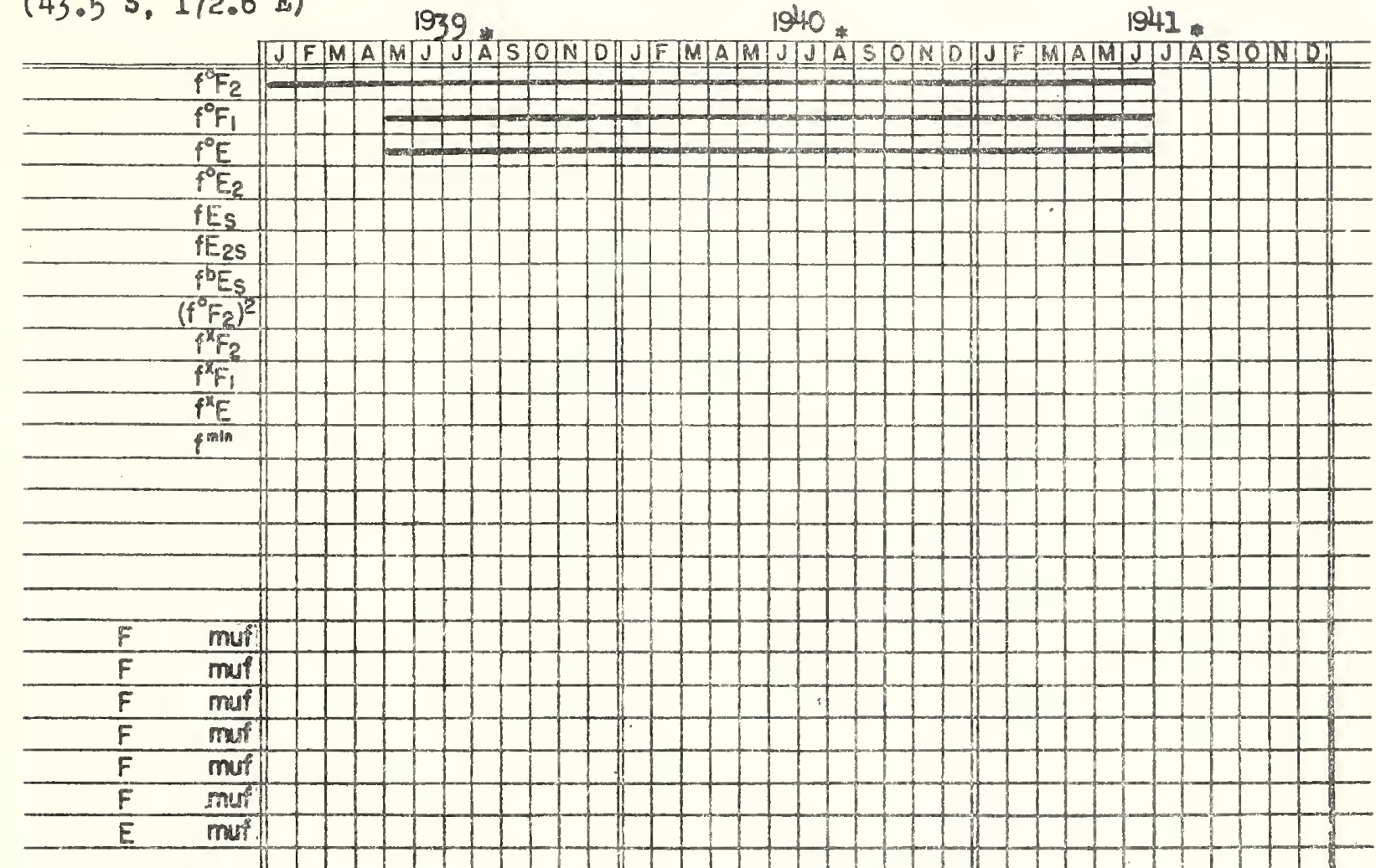

$\frac{M 3500}{M 3000}$

* Fa M3000

* Fr M4000

$h F_{2}$
$h F_{1}$

KE

$K E_{2}$

$h^{\prime} E_{s}$

$h^{\prime} E_{32}$

$h^{m i} F_{2}$

$h^{\pi} F_{1}$

$h^{m} E$

Absorptlon

* Colculated by IRPL

* 11 data from Jan. 1939 through

July 1942 monthly averages. 

Chricichurc IONOSPHERIC DATA RECORD

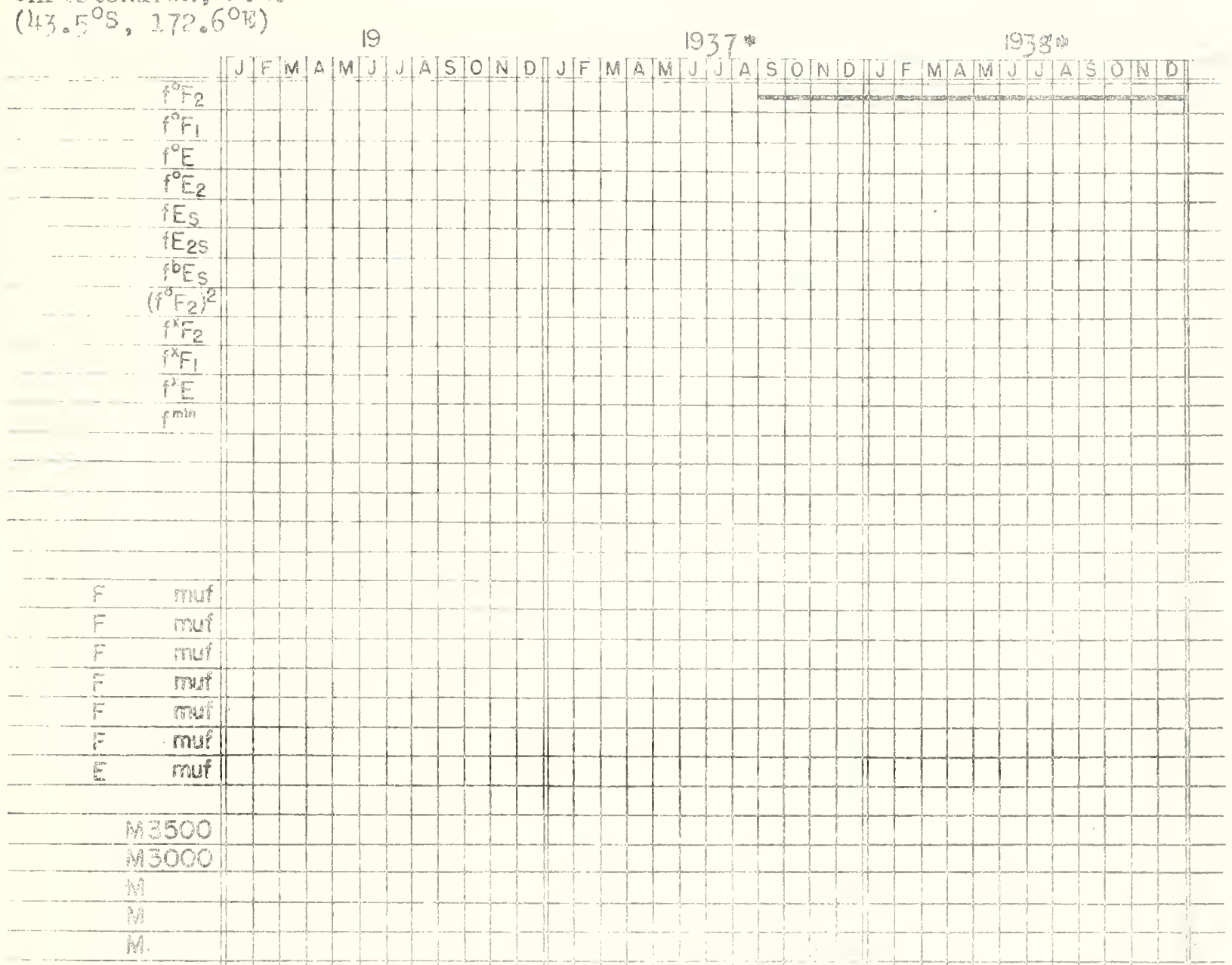

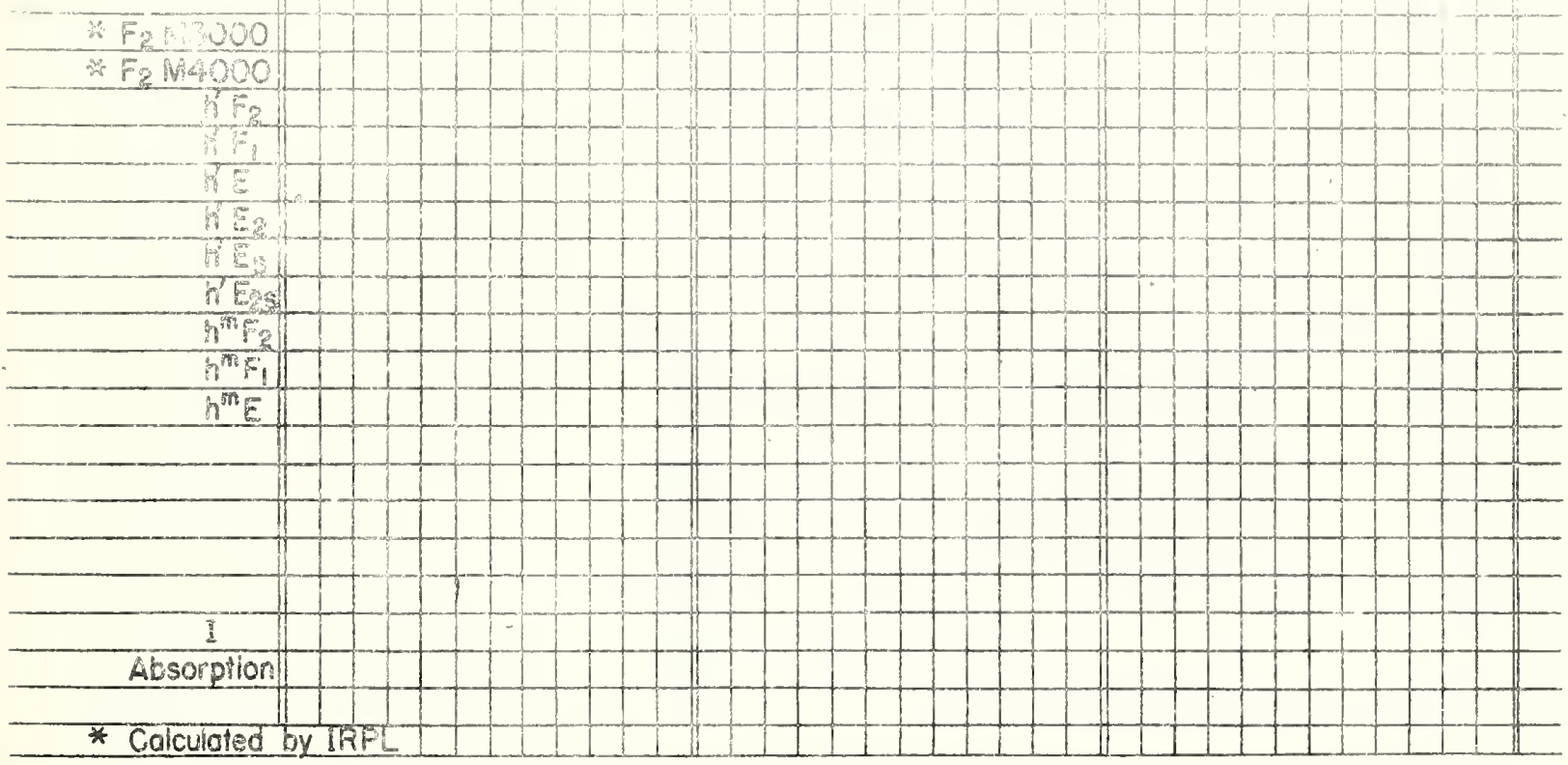

-All data through JuIy 1942 monthly averiges. 

Christmes 1.

$\left(1.9^{\circ} 1,157.3^{\circ}\right.$ \%)

\section{IONOSPHERIC DATA RECORD}

1944

1945

19

\begin{tabular}{|c|c|}
\hline \multicolumn{2}{|c|}{$F_{23} 000 m$ if } \\
\hline$F$ & muf \\
\hline$F$ & muf \\
\hline$F$ & muf \\
\hline$F$ & muf \\
\hline$F$ & muf \\
\hline$E$ & muf \\
\hline
\end{tabular}

M3500

$\mathrm{M} 3000$

$M$

- M
* $\mathrm{F}_{2} \mathrm{M} 3000$

* $F_{2} M 4000$

$h^{\prime} \mathrm{F}_{2}$

${ }^{\prime} F_{1}$

TE

$h^{\prime} E_{2}$

$h^{\prime} E_{S}$

$h^{\prime} E_{25}$

$h^{m} F_{2}$

$h^{\text {m }} F_{1}$

$h^{m} E$

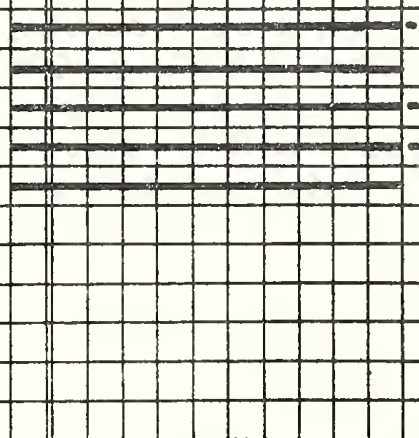



Chungling. China

(29.40\%, 106.80
IONOSPHERIC DATA RECORD

1945
19
19

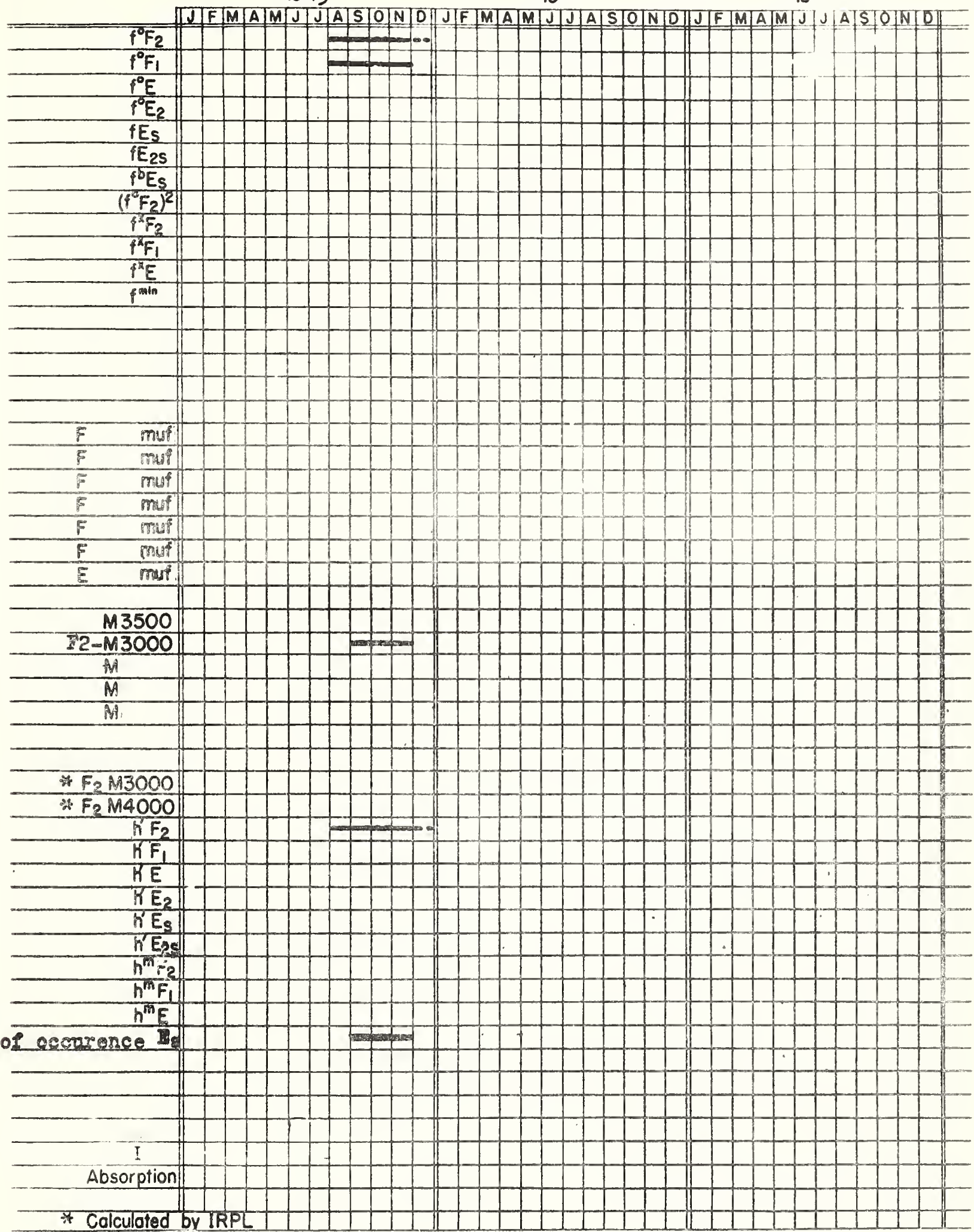





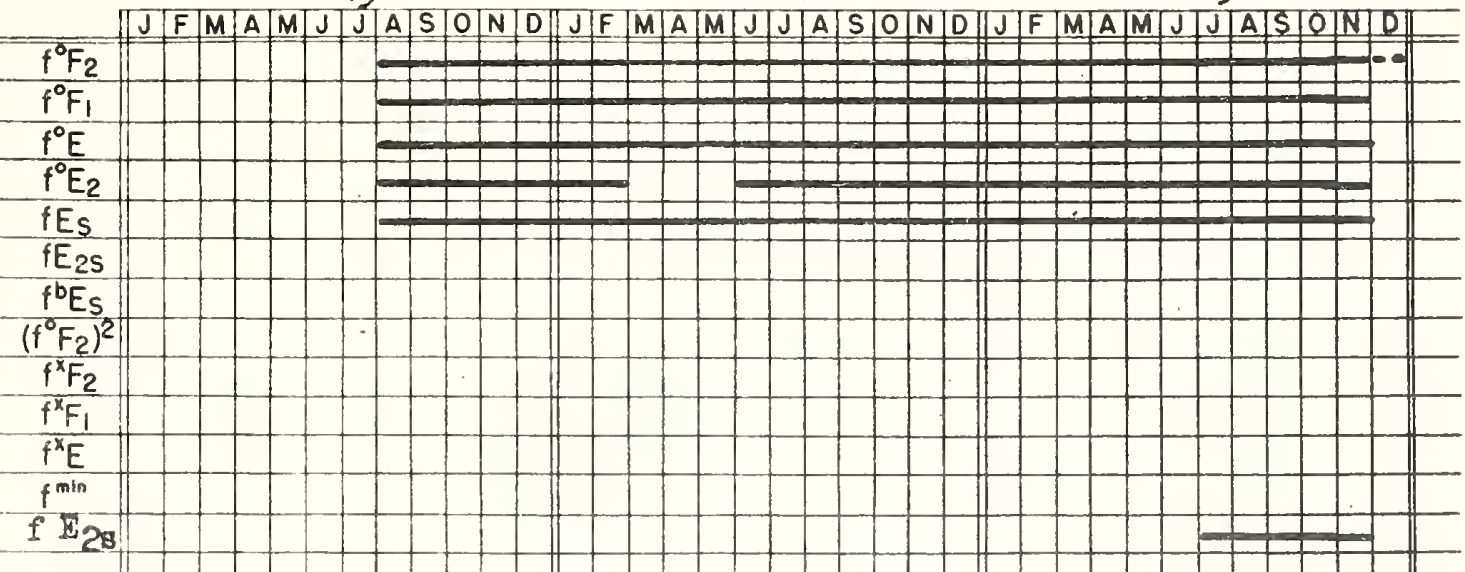

\section{$F_{2} 3500$ muf $F$ mut F muf F muf $F_{1} 3000$ muf $F$ muf E 1500 muf}

M3500

R2 13000

F1 M 1500

FI-M 3000

I $M 1500$

* Fr M3000

* Fr M4000

\begin{tabular}{c|}
$h^{\prime} F_{2}$ \\
\hline$h^{\prime} F_{1}$ \\
\hline$h^{h} E$ \\
$h^{\prime} E_{2}$ \\
$h^{\prime} E_{5}$ \\
$h^{\prime} E_{25}$ \\
$h^{m} F_{2}$ \\
$h^{m} F_{1}$ \\
\hline$h^{m} E$
\end{tabular}

WTV Recopt10in

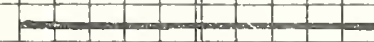

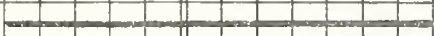
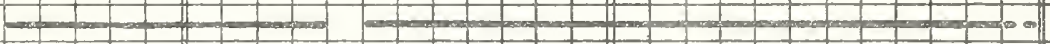
$-1-1-1-1.5-10$

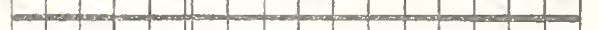
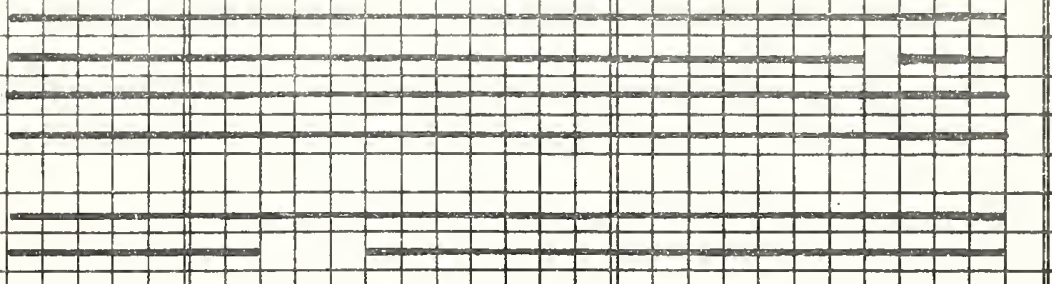



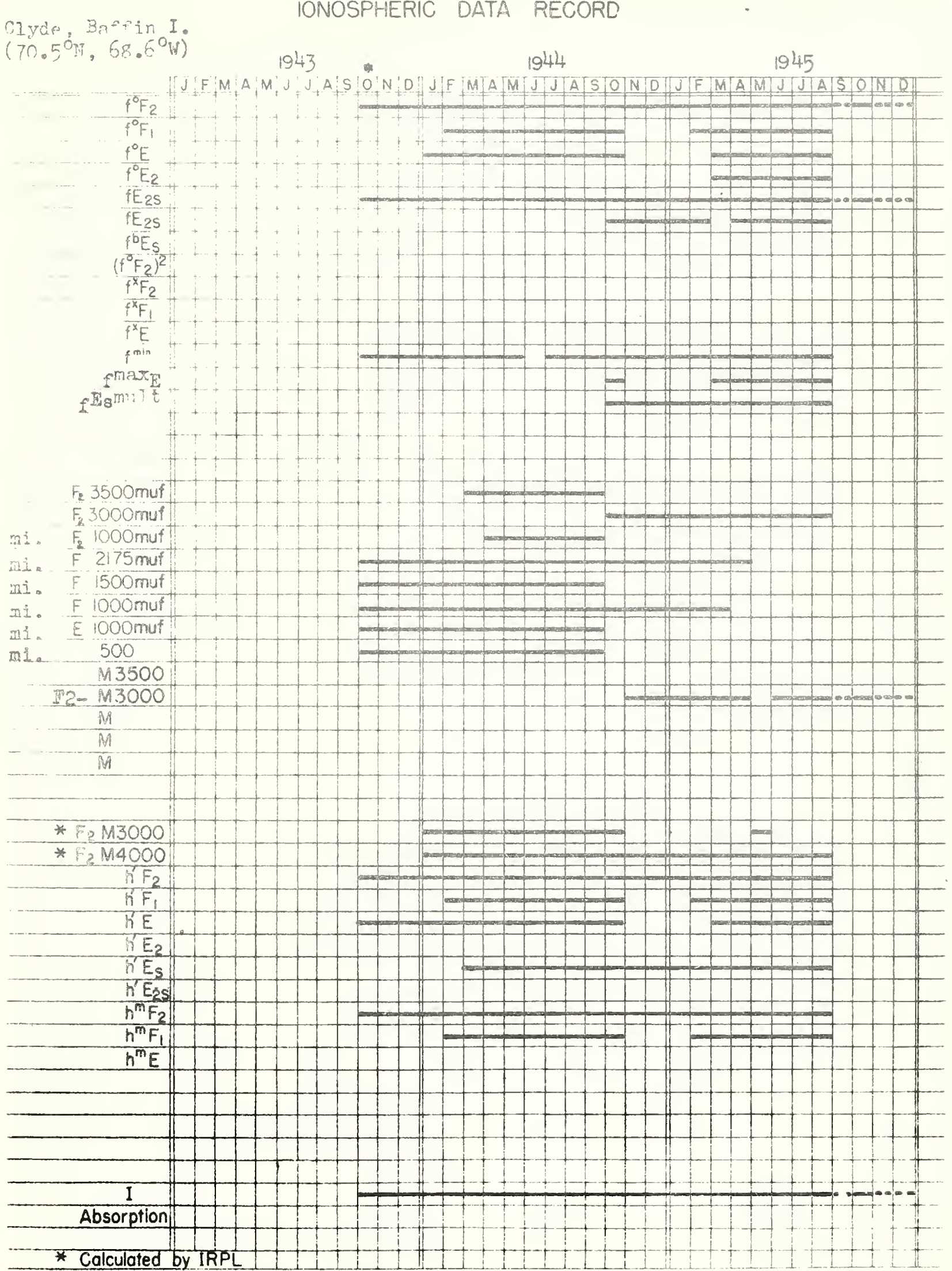

-Oct. 1943.8 days only. 

Colombo, Cevion

$\left(6.6^{\circ}, 80.0^{\circ} \mathrm{E}\right)$
IONOSPHERIC DATA RECORD

1945

19

19

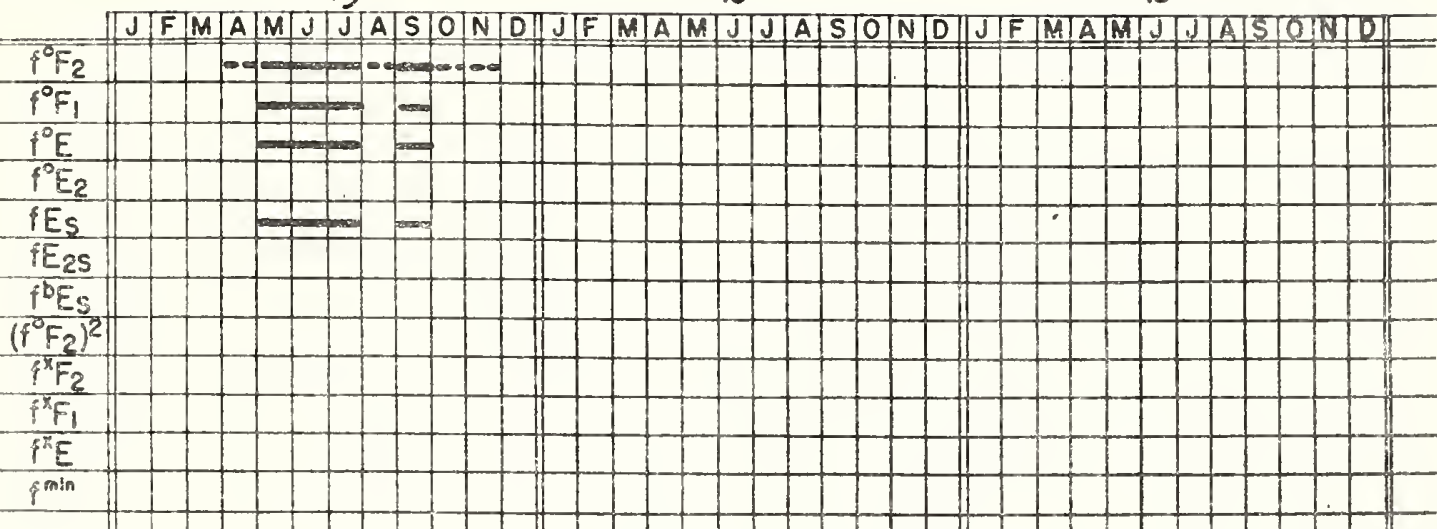

- mú

fo mut

5 mut

is mut

F Tui

E mot

M3500

s. $2 \cos ^{2} 3000$ im 

Deal, New Jersey

$40.2 \circ \mathrm{x}, 740 \mathrm{~W}$
IONOSPHERIC DATA RECORD

1939

19

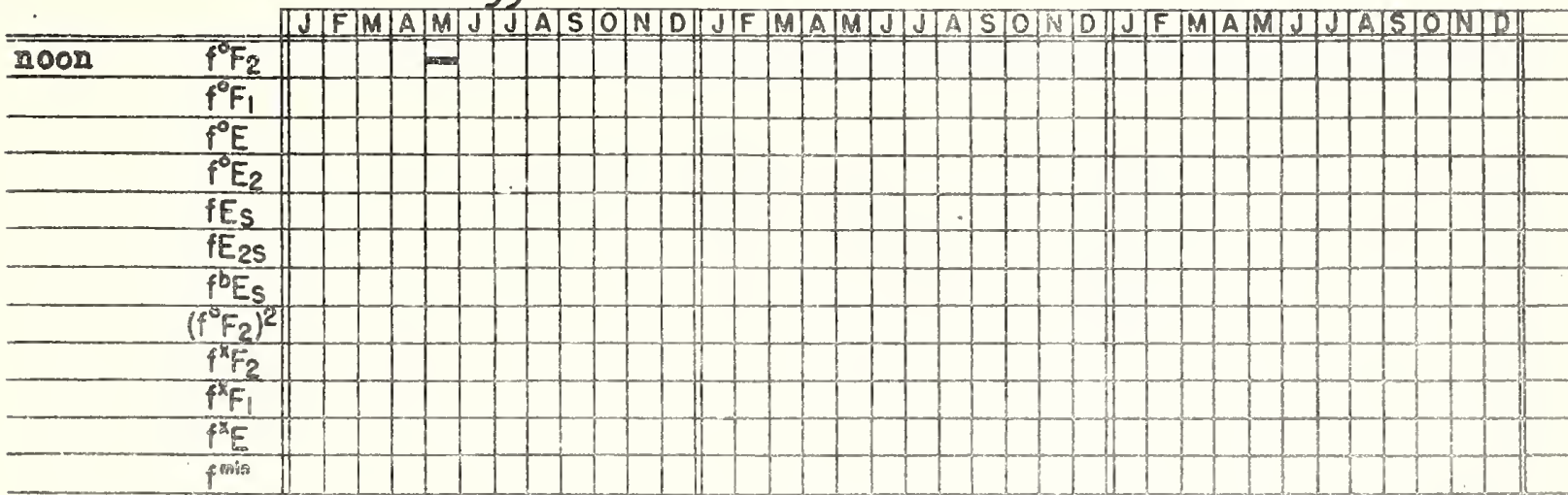

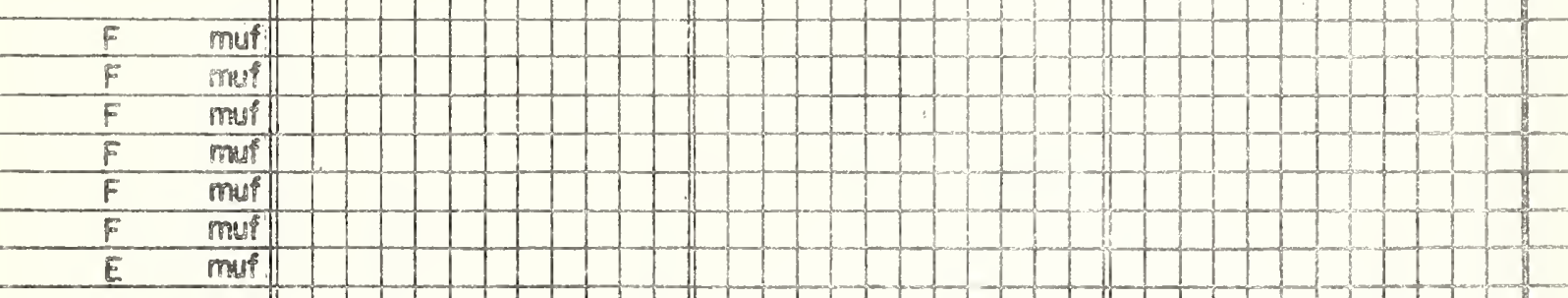

$\frac{M 3500}{M 3000} \frac{M}{M}$

* F $M 3000$

* F $M 4000$

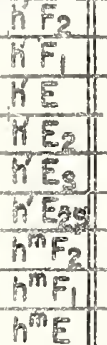

(1)

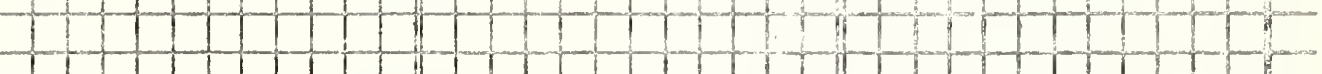

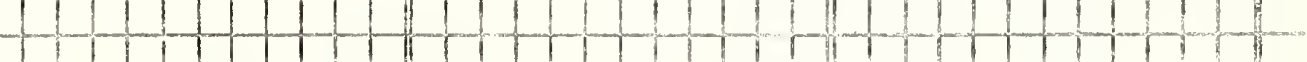

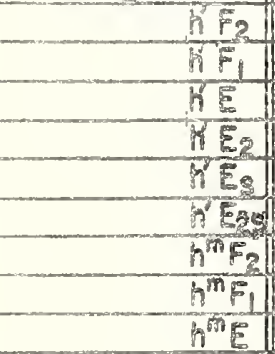

1

I

Absorption

Calculated by IRP 


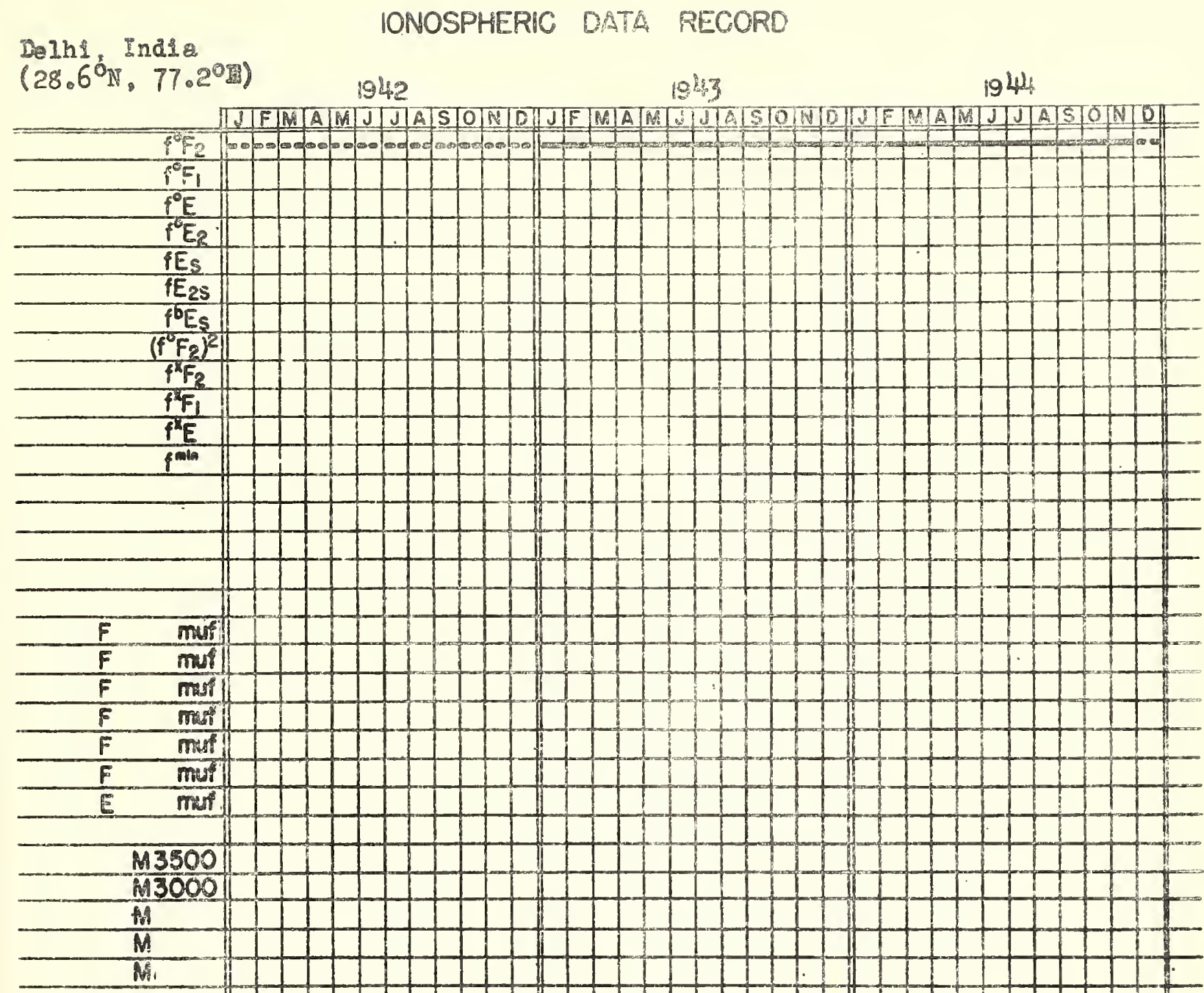

\section{Delhi. Indse \\ (19: \\ 1942 \\ $10 \% 3$ \\ 1944}

F.

$f^{\circ} \mathrm{E}$

SEs

PELS

$\left(f^{6} \mathrm{~F}\right.$ )

$f^{x} F_{2}$

$f^{x} E$

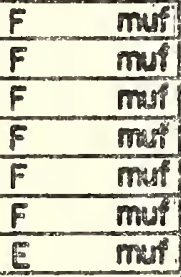

M3500

Ni

* FrM3000

* Fr M4000

$\mathrm{hF}_{2}$

hF

KE

HE:

$f^{\prime} E_{3}$

$h^{\prime} \mathrm{s}_{\mathrm{s}}$

$h^{h^{m} F_{2}}$

$h^{m} E$

Absorption

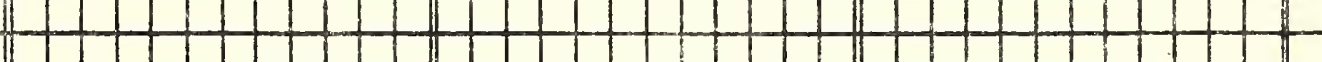

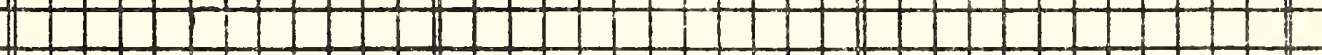

Caculed 
Delht, India

$\left(28.6 \delta_{N}, 77.2^{\circ \mathrm{g}}\right)$
IONOSPHERIC DATA RECORD

$1945 \quad 19$

19

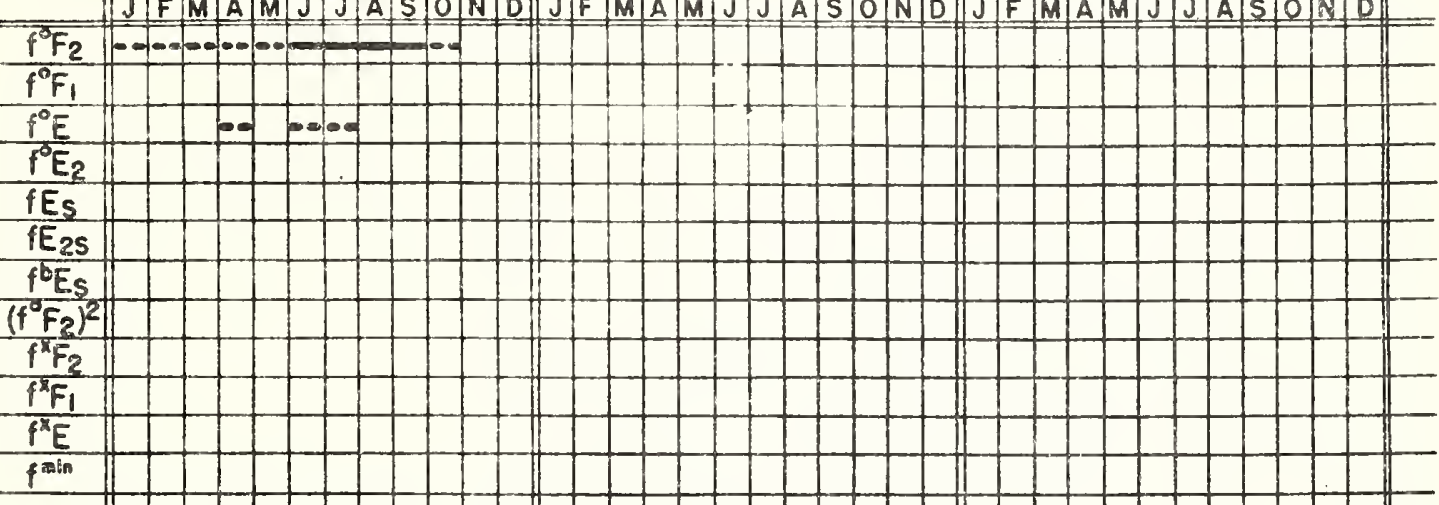

\begin{tabular}{rr|}
\hline$F$ & muf \\
\hline$F$ & muf \\
\hline$F$ & muf \\
\hline$F$ & muf \\
\hline$F$ & muf \\
\hline$F$ & muf \\
\hline$E$ & muf
\end{tabular}

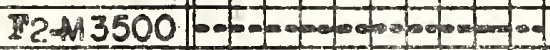

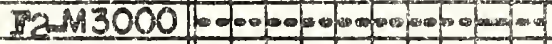

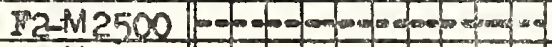

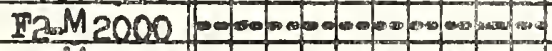

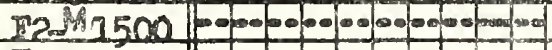

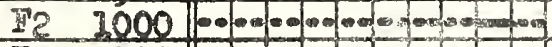

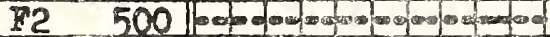

* Fo M 3000

* F MP MCOO

TF2 I Dol

if

RE

B.

n

$\pi \varepsilon^{2}$

$h^{\text {m }}$

$h^{\text {nif }} F_{1}$

$h^{\text {ne }}$

hP2 at .831P2

22 at 9172

his at 0950 to

bI2-at - 9750 ?

LF2 at . $95 \mathrm{fP}^{2}$

I

Absorption

* Calculated by IRP

Tabulations forz and hre

June 11 through June 21

July 11 through July 20

Aug. 13 throush Axe. 20 

Fairbanks, Alaslea

$\left(64.9^{\circ} \mathrm{N}, 147.3^{\circ} \mathrm{W}\right)$

1944

1945

19

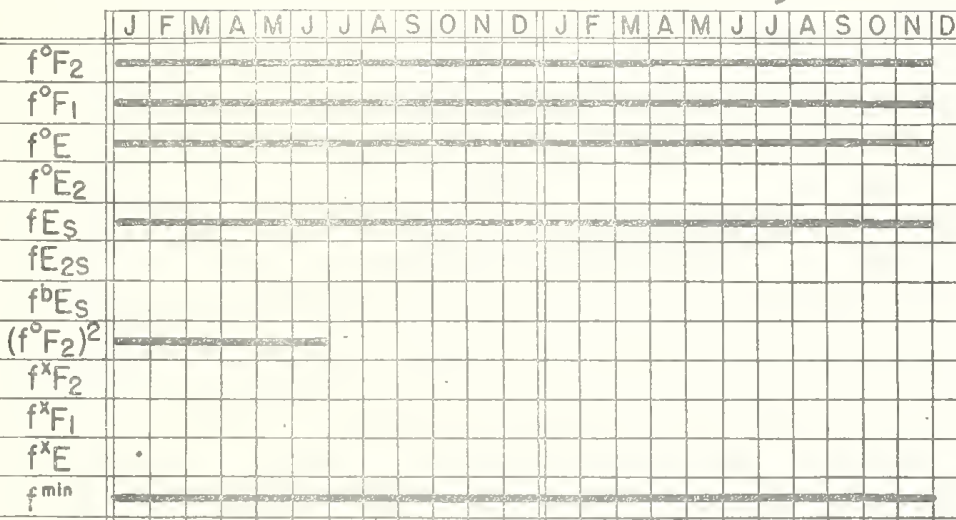

$F_{2} 3500$ muf $=-1$

$\mathrm{F}_{2} 3000 \mathrm{muf}$

$F_{3} 1500$ muf

$\mathrm{F}_{2}$ IOOOmuf enm

$\mathrm{F}_{2}$ 500muf

$F_{2}$ 250muf

E muf

F2 - M 3500 -

F2 - M3000

$\mathrm{M}$

$\mathrm{M}$

S2-M1000

* $F_{2} M 3000$ -

* $\mathrm{F}_{2} \mathrm{M} 4000-1 \mathrm{n}$

$h^{\prime} F_{2}=-2-1-5$

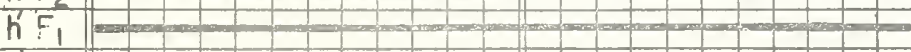

h'E

$h^{\prime} E_{2}$

$h^{\prime} E_{S}$

$h^{\prime} E_{2 s}$

$h^{m} F_{2}=-1-2$

$h^{m} F_{1}-1$ -

$h^{m} E$

Fade outs 

Fairbanks, Hzaska

IONOSPHERIC DATA RECORC

$\left(64.9^{\circ} \mathrm{N}, 147.80 \mathrm{~W}\right)$

1942

1942

1943

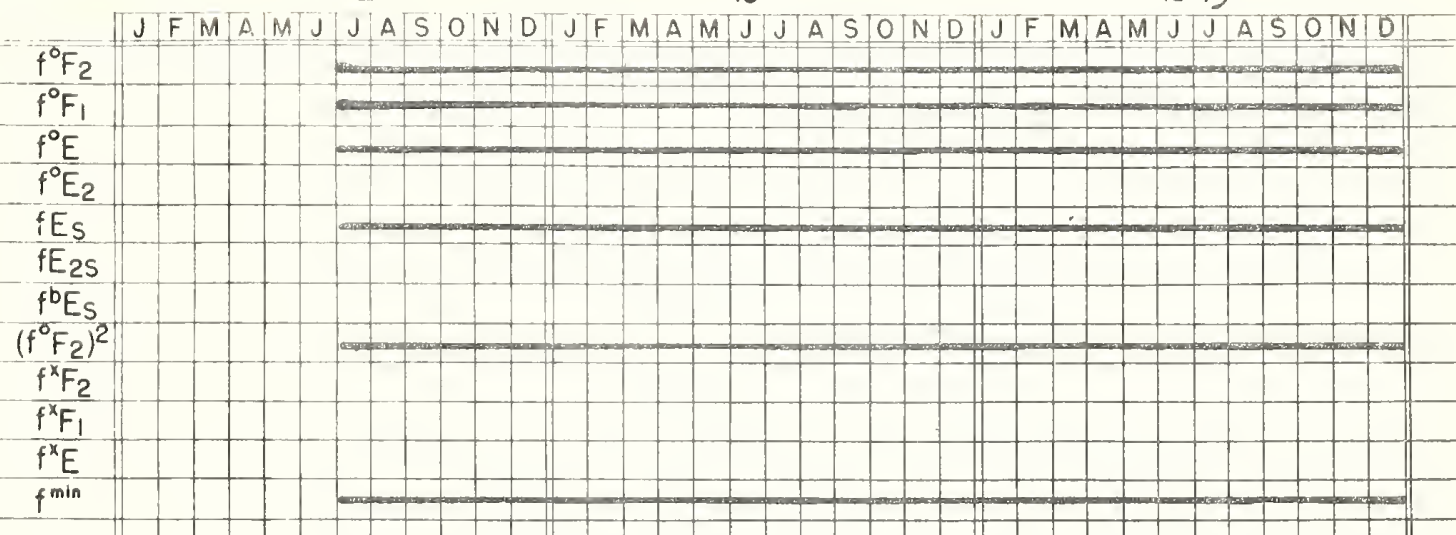

\begin{tabular}{|c|c|}
\hline$F$ & 3500 muf \\
\hline$F$ & 3000 muf \\
\hline$F$ & 1500 muf \\
\hline$F$ & 1000 muf \\
\hline$F$ & 500 muf \\
$F$ & 250 muf
\end{tabular}

F2 3500muf

F2 1500

F2 $-M 3500$

M3000

$M$

F2-M1000

* $F_{2} M 3000$

* $\mathrm{F}_{2} \mathrm{M} 4000$

$\mathrm{h}^{\prime} \mathrm{F}_{2}$

$h^{\prime} F_{1}$

h'E

$h^{\prime} E_{2}$

$h^{\prime} E_{S}$

$h^{\prime} E_{2 S}$

$h^{m} F_{2}$

$h^{m} F_{1}$

$h^{m} \mathrm{E}$

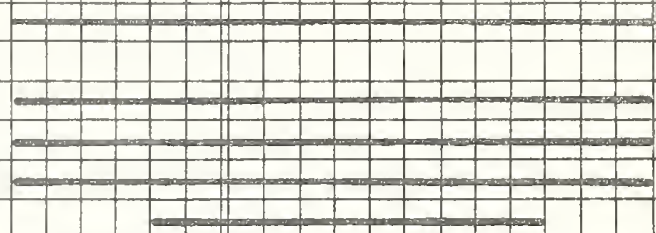

Fadeouts

no $\mathrm{F}_{2}$ layer reflect

I

Absorption

$\mathrm{T}_{2}$ scattering inde $\mathrm{x}$

* Calculated by IRPL

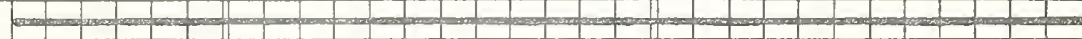

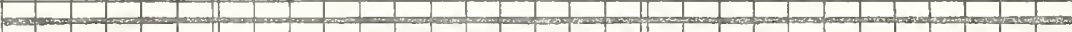

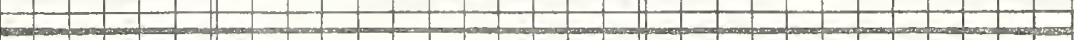

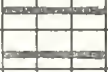

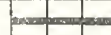

rear 

Great Baddow Fngland

$\left(51.7^{\circ} \pi, 0.5^{2}\right)$

IONOSPHERIC DATA RECORD

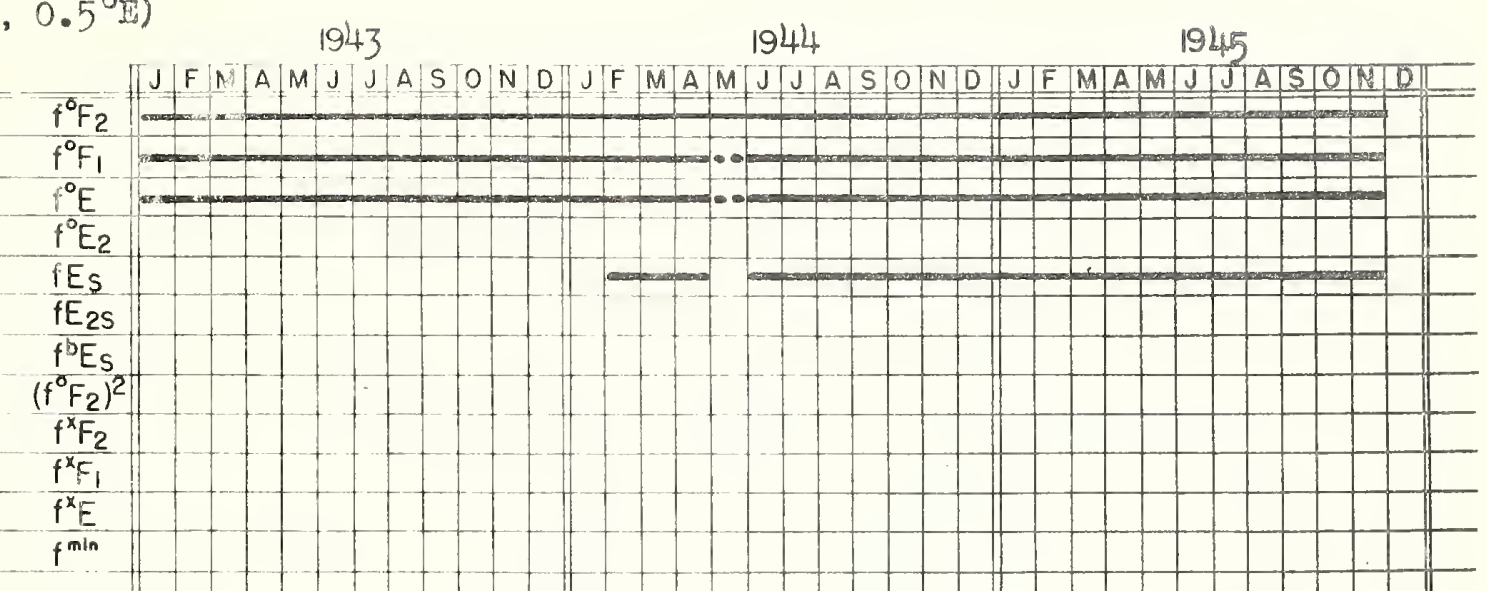

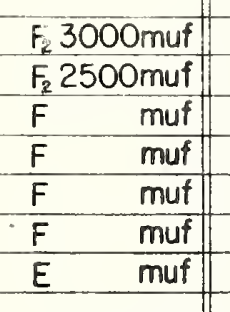

M3500

T2 $-M 3000$

$\frac{M}{M} 3000$

ए 1500

$M 2500$

* $\mathrm{F}_{2} \mathrm{M} 3000$

* $\mathrm{F}_{2} \mathrm{M} 4000$

\begin{tabular}{|c|}
\hline$h^{\prime} F_{2}$ \\
$h^{\prime} F_{1}$ \\
$h^{\prime} E_{1}$ \\
$h^{\prime} E_{2}$ \\
\hline$h^{\prime} E_{s}$ \\
$h^{\prime} E_{2 s}$ \\
$h^{m} F_{2}$ \\
\hline$h^{m} F_{1}$ \\
$h^{m} E$
\end{tabular}

I

Absorption

* Calculated by IRPL
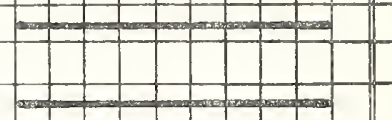

Groat Baddow, England IONOSPHERIC DATA RECORO

$\left(51.7^{\circ} \mathrm{N}, 0.5^{\mathrm{O}} \mathrm{E}\right)$

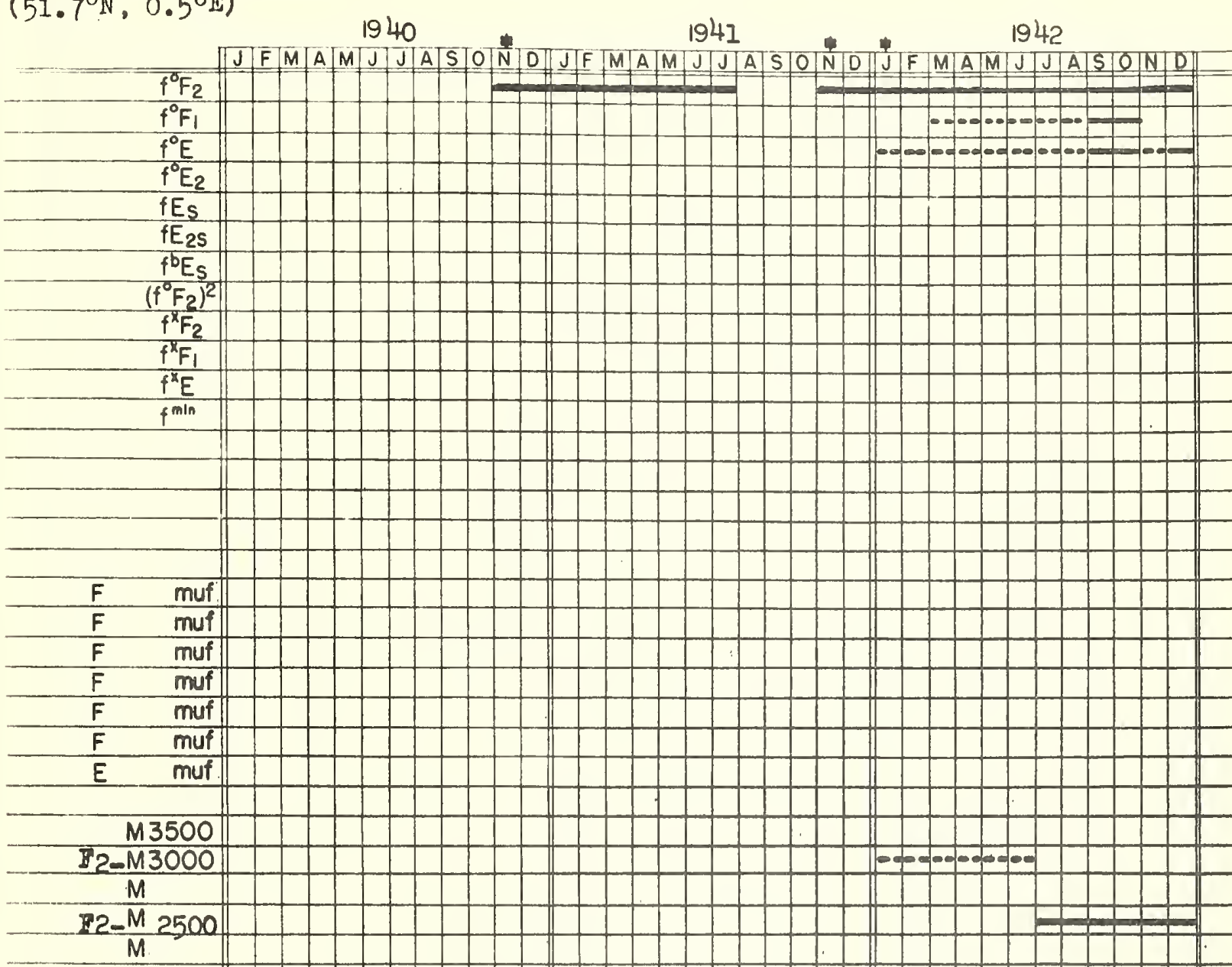

* $F_{2} M 3000$

* $\mathrm{F}_{2} \mathrm{M} 4000$

$\mathrm{h}^{\prime} \mathrm{F}_{2}$

RE

$h^{\prime} E_{2}$

$h^{\prime} E_{s}$

$h^{\prime} E_{2 s}$

$h^{m} F_{2}$

$h^{m} F_{1}$

$h^{m} E$

Absorption

* Colculated by IRPL

*Nov. 1940 throush July 1944, read from graphs.

Monthly averifes by mail through Dec. 1941.

Daily-hourly tabulations since Jan. 19 lt2. 
Guem I.

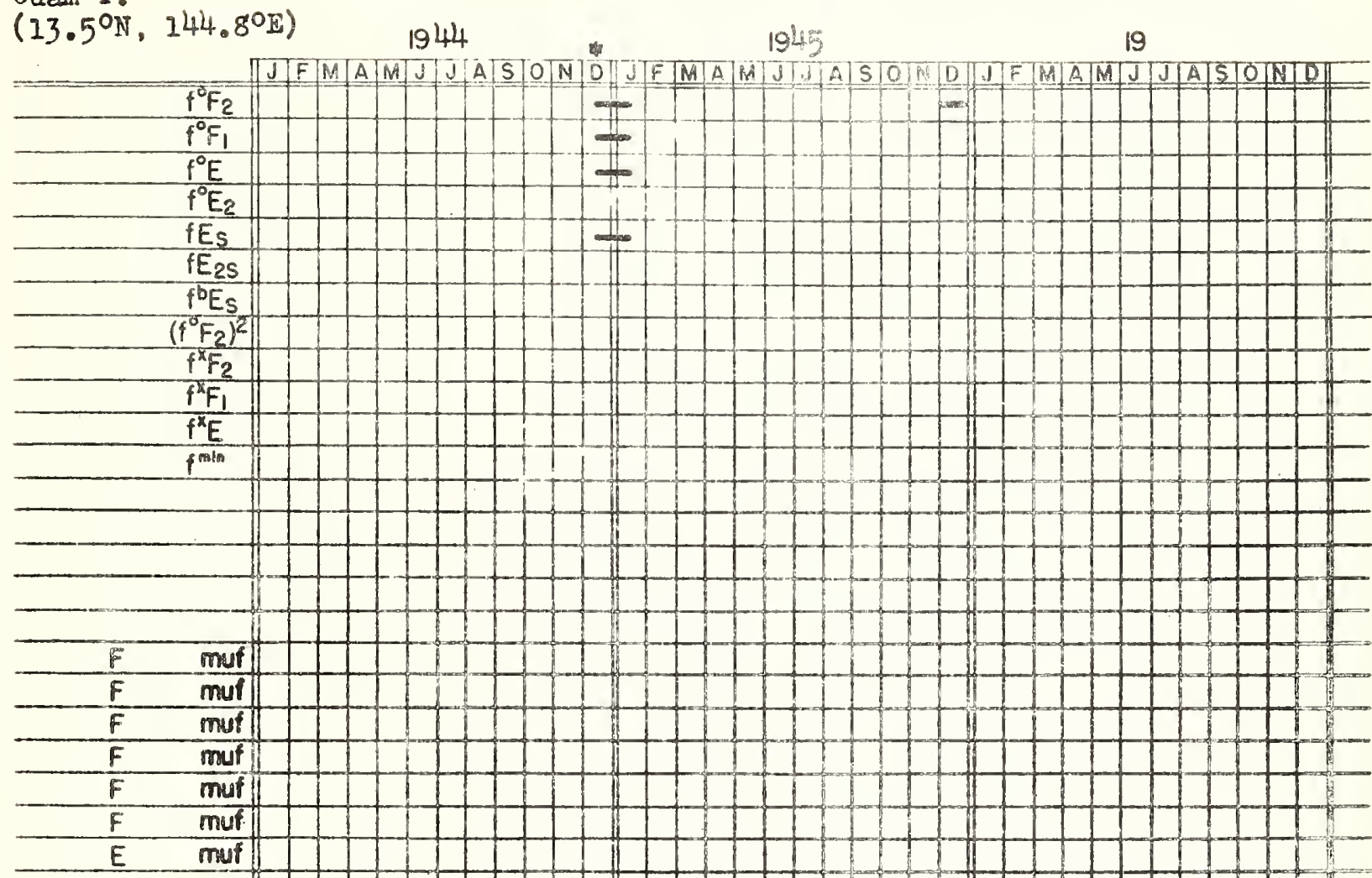

M3500

12 $M 3000$

$M$

$-\infty$

M

M

* $F_{2} M 3000$

* $F_{2} M 4000$

$h^{\prime} F_{2}$

h' $F$

TE

तE

$h^{\prime} E_{s}$

$h E_{2}$

$h^{m} F_{2}$

$\frac{h^{m} F_{1}}{h^{m} E}$

I

Absorption

* Calculated by IRPL

- December 27 through January 6 only. Dallywhourly tabulations. 

Hobart, Tasmania

$\left(42.9^{\circ} \mathrm{S}, 147.3^{\circ} \mathrm{E}\right)$

IONOSPHERIC DATA RECORD

1945

19

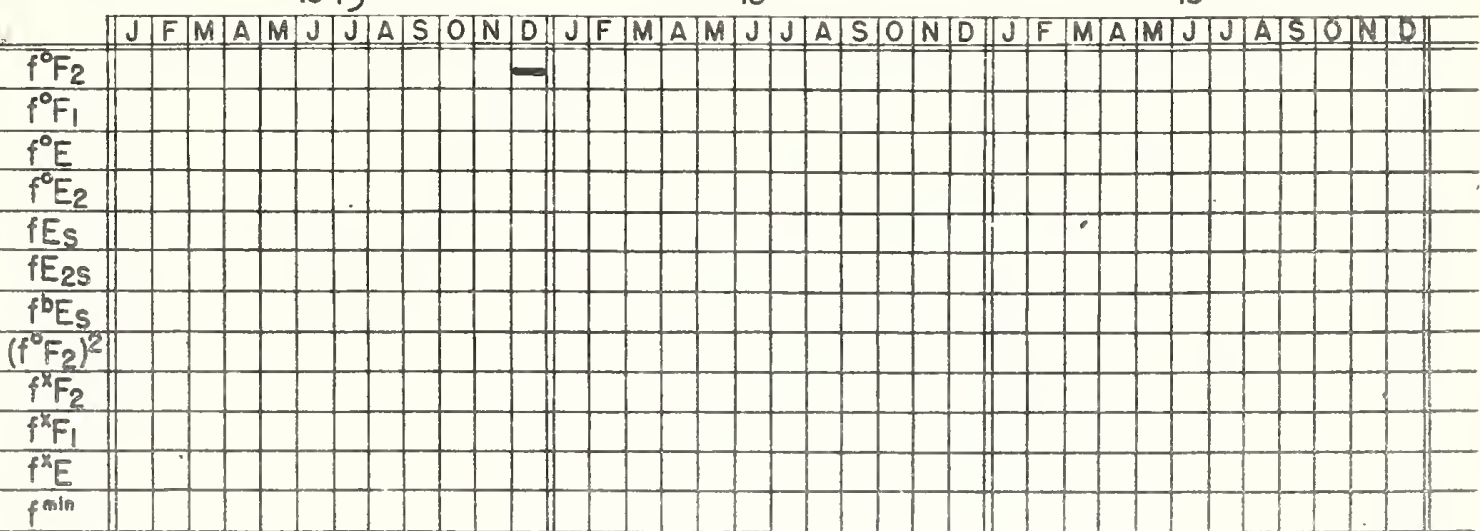

$M 3500$
$M 3000$
$M$
$M$

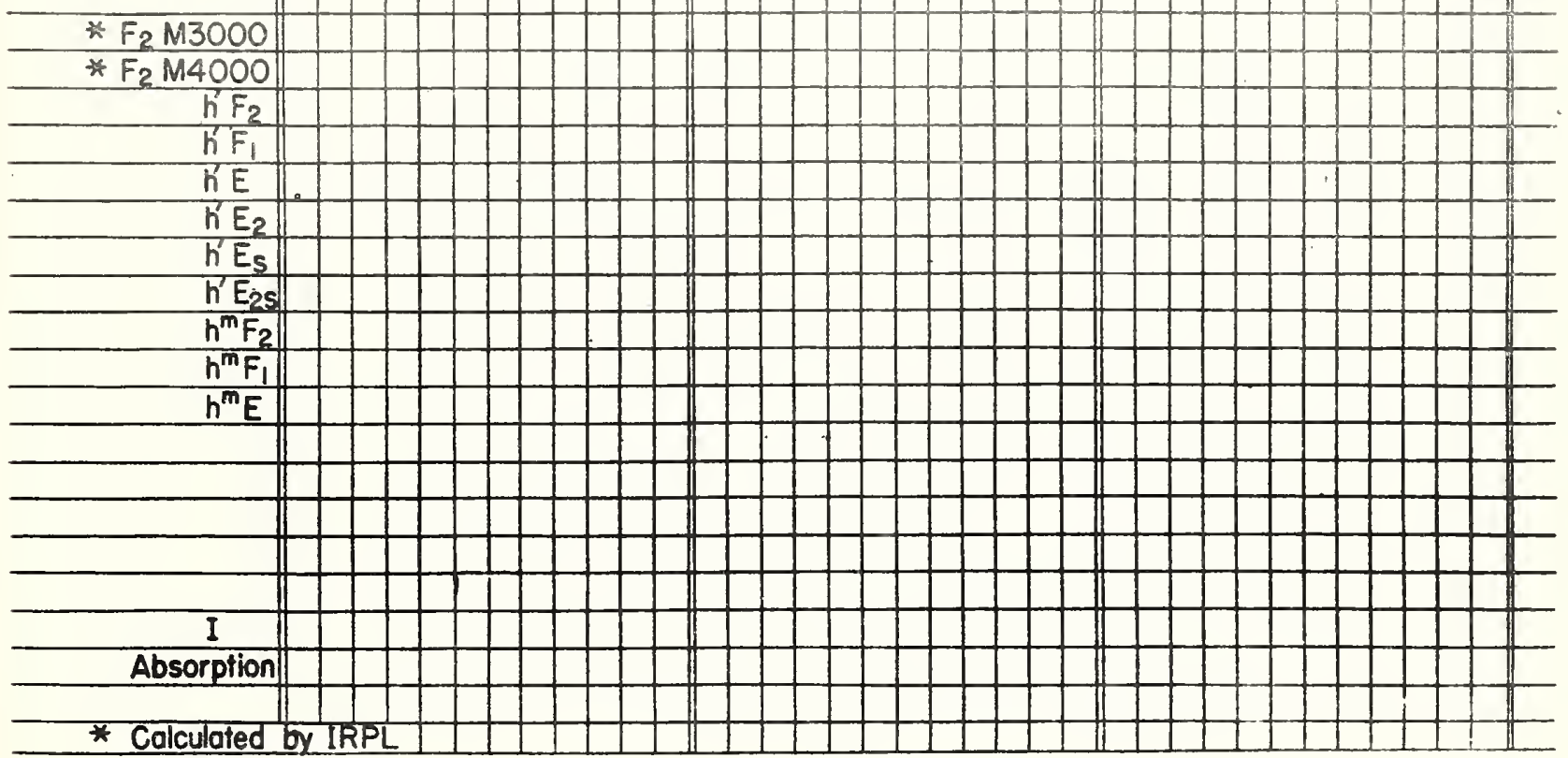



Hollandia, New Guinea.

(3.00\%, $140.00 \mathrm{E})$
IONOSPHERIC DATA RECORD

1945

19

19

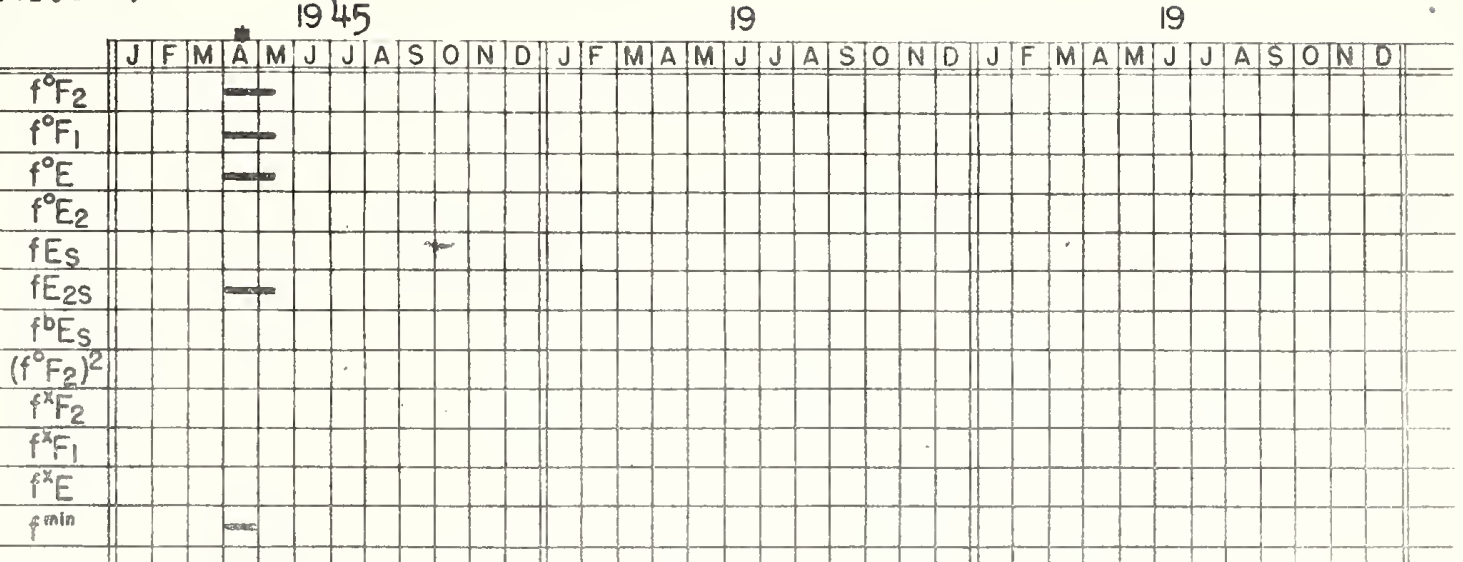

\begin{tabular}{|c|c|}
\hline \multicolumn{2}{|c|}{$\mathrm{F}_{2} 3000 \mathrm{muf}$} \\
\hline$\vec{F}$ & muf \\
\hline$F$ & muf \\
\hline$F$ & muf \\
\hline$F$ & mut \\
\hline$F$ & muf \\
\hline$E$ & muf \\
\hline & 500 \\
\hline पृ? & 000 \\
\hline & \\
\hline की? & 4000 \\
\hline
\end{tabular}

M3500

In $2-M 3000$

M

T.2-M 4000

$+1$

\section{$+1 .+1$}

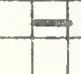

* F $F_{2} 13000$

* $\mathrm{F}_{2} \mathrm{M} 4000$

$\mathrm{h}_{2}$

$h^{\prime} F_{1}$

hE

$h^{\prime} E_{2}$

$h^{\prime} \bar{E}_{S}$

$h^{\prime} E_{2 s}$

$h^{m} F_{2}$

$h^{m} F_{1}$

$h^{m} E$ 

Hon shu, Japan

IONOSPHERIC DATA RECORO

$\left(35.3^{\circ} \mathrm{N}, 135.0^{\circ} \mathrm{F}\right)$

1943

\begin{tabular}{l}
1943 \\
19 \\
$f^{\circ} \mathrm{F} 2$ \\
\hline
\end{tabular}

\begin{tabular}{l}
1943 \\
19 \\
$f^{\circ} \mathrm{F} 2$ \\
\hline
\end{tabular}

$f^{\circ} F_{1}$

$f^{\circ} E$

fEs

$f E_{25}$

${ }^{b} E_{S}$

$\left(f^{\circ} \mathrm{F}_{2}\right)^{2}$

$f^{x} F^{i}$

$f^{x} E$

$f^{\text {min }}$

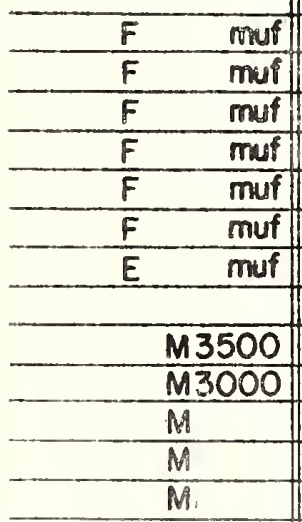

$* F_{2} M 3000$

* Fr M4000

\begin{tabular}{l|}
\hline$h^{\prime} F_{2}$ \\
$h^{\prime} F_{1}$ \\
$h^{\prime} E$ \\
$h^{\prime} E_{2}$ \\
\hline$h^{\prime} E_{s}$ \\
$h^{\prime} E_{2 s}$ \\
$h^{m} F_{2}$ \\
$h^{m} F_{1}$ \\
$h^{m} E$ \\
\hline$I$
\end{tabular}

Absorption

* Calculated by IRPL

\section{Graphs only.}





\section{(12.0 $\left.\mathrm{S}, 75.3^{\circ} \mathrm{W}\right)$}

1943

1944

1945

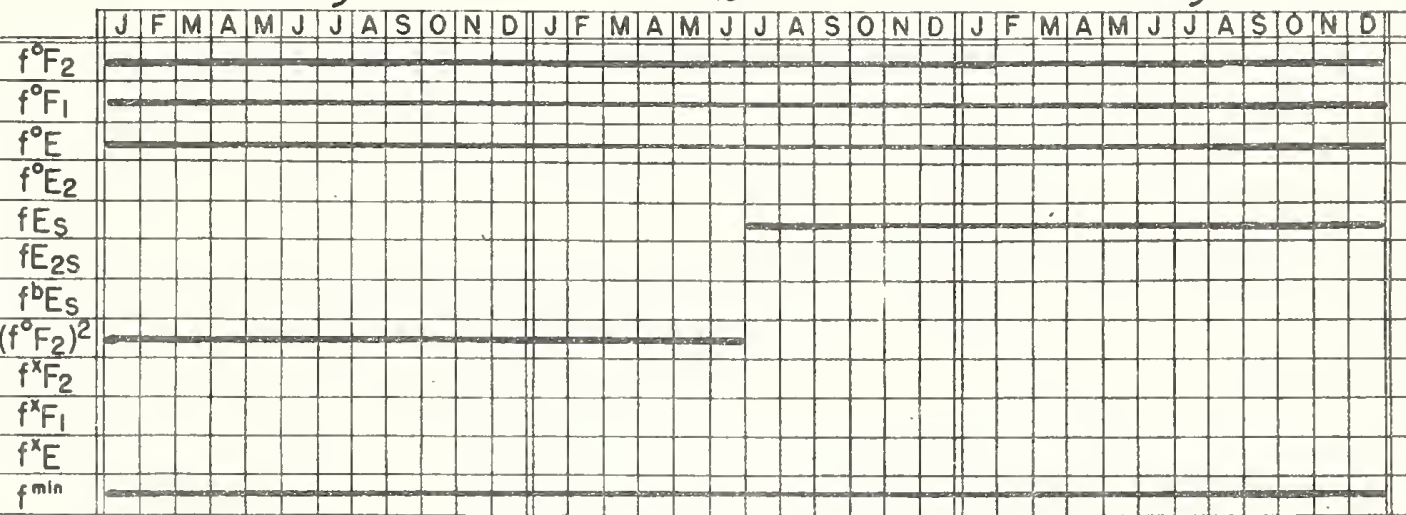

$F_{2} 3500$ muf

$F_{2} 3000$ muf

$F_{2}$ 1500muf

$F_{2} 1000$ muf

$\mathrm{F}_{2}$ 500muf

$F_{1}$ muf

$E$ muf

$M 3500$

F2-M3000

$M$

* $F_{2} M 3000$

* $\mathrm{F}_{2} \mathrm{M} 4000$

$h^{\prime} F_{2}$

$\mathrm{h}^{\prime} \mathrm{F}_{1}$

$h^{\prime} E$

$h^{\prime} E_{2}$

$h^{\prime} E_{S}$

$h^{\prime} E_{25}$

$\mathrm{h}^{\mathrm{m}} \mathrm{F}_{2} \longrightarrow$

$\mathrm{h}^{\mathrm{m}} \mathrm{F}_{1} \rightleftharpoons$ L

$h^{m} E$

Absorption

* Calculated by IRPL

To July 1, 1940, Monthly averages only.

From July 1, 1940 dally-hnurly tabulations. 


Fin: ncryo, Pema
IONOSPHERIC DATA RECORD
$\left(12.005,75.3^{\circ} \mathrm{W}\right)$
1940
1941
1942

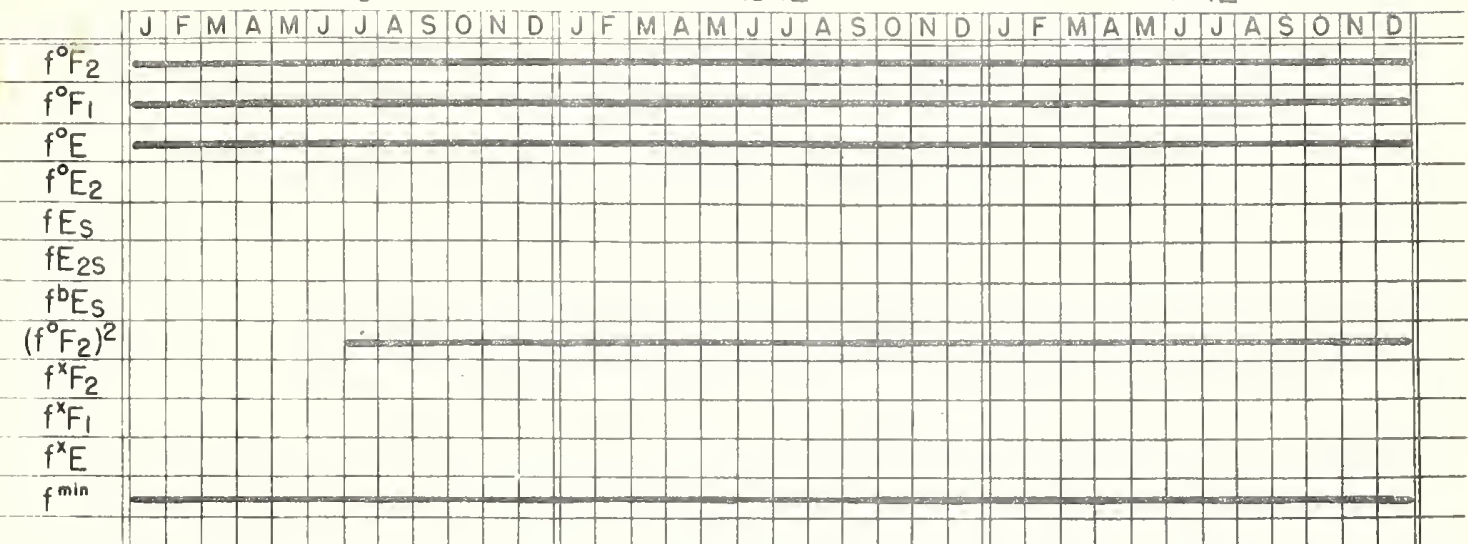

\footnotetext{
$F_{2} 3500$ muf

mi. $\quad F_{2} 1500$ muf

mi. $\quad F_{2} 1000$ muf

mi. $F_{2} 500$ muf

$F_{1}$ muf

$F_{1} \quad$ muf

$E$ muf

M3500

M3000

M
}

* $\mathrm{F}_{2} \mathrm{M} 3000$

* Fr M4000

$h^{\prime} F_{2}$

h'F

h'E

$h^{\prime} E_{2}$

$h^{\prime} E_{S}$

$h^{\prime} E_{2 S}$

$h^{m} F_{2}$

$h^{m} F_{1}$

I

Absorption

* Calculated by IRPL

To July 1, 1940, monthly averages only.

Since July 1, 1940, dalimenorriy tabulations. 


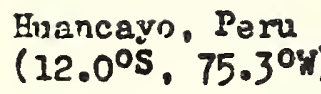

\section{IONOSPHERIC DATA RECORO}

\section{$1937 \quad 1938$}

1939

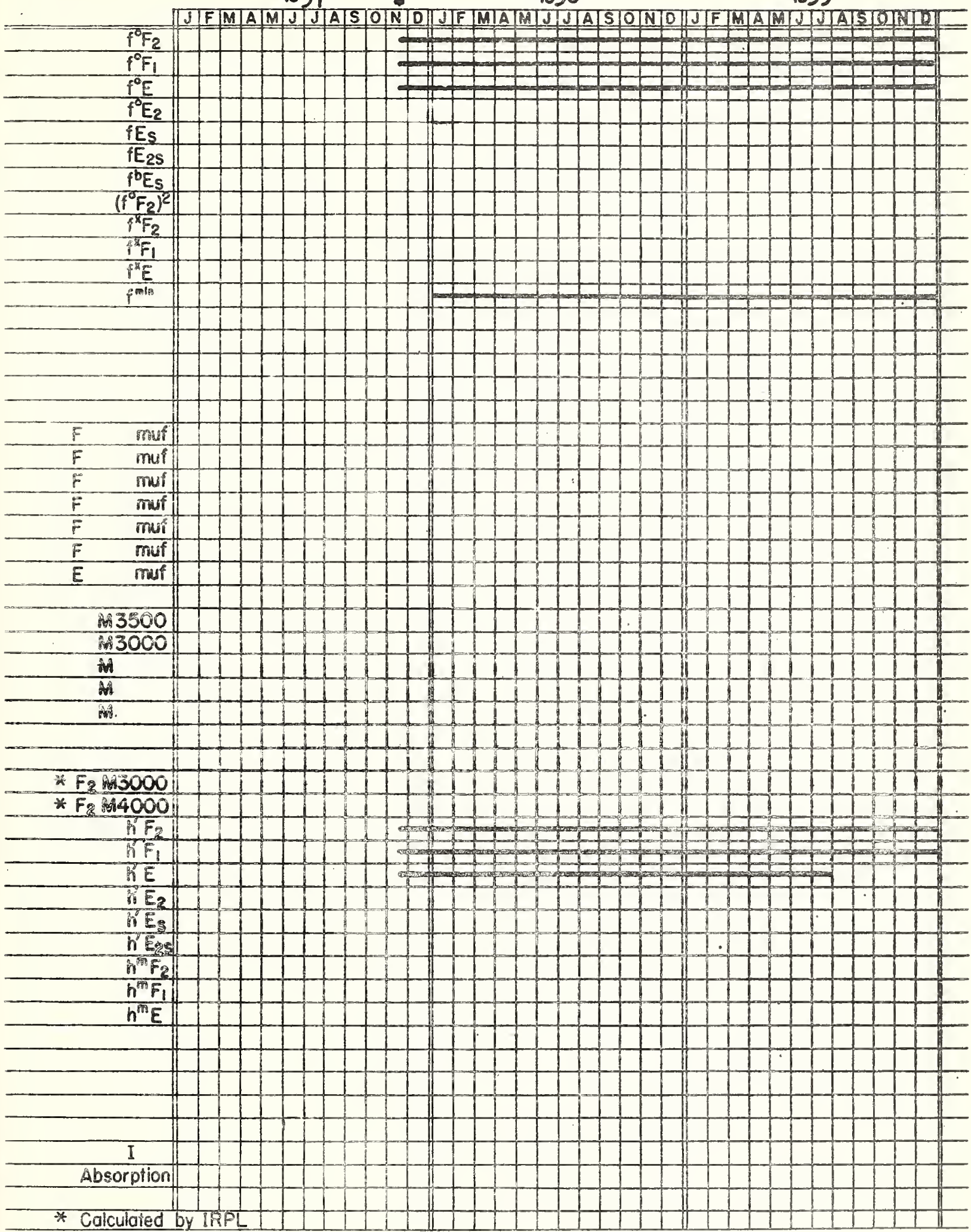

*Nov. 1937, 17 to 30 only.

To July I, 1940, monthly averages only.

Since July I. 1.940 dally-hour Iy tabulations. 



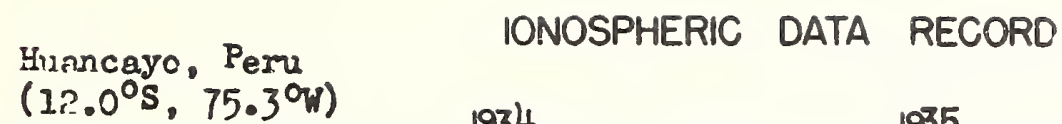

\section{$\left(12.0^{\circ} \mathrm{S}, 75.3^{\circ} \mathrm{W}\right)$ \\ 1934 \\ 1935 \\ 1936}

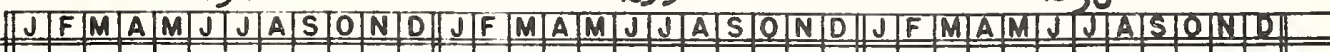

$f^{\circ} F_{2}$
$f^{\circ} F_{1}$
$f^{\circ} E$
$f^{\circ} E_{2}$
$f E_{S}$
$f E_{2 S}$
$f^{b} E_{S}$
$\left(f^{\circ} F_{2}\right)$
$f^{x} F_{2}$
$f^{2} F_{1}$
$f^{x} E_{1}$
$f^{\text {min }}$

बी

...
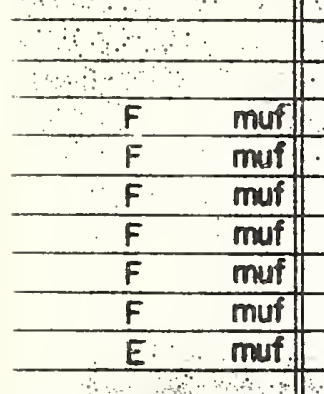

$\frac{M 3500}{M 3000} \frac{M}{M}$

* $\mathrm{F}_{2} \mathrm{M} 3000$

* $F_{2} M 4000$

$h^{\prime} F_{2}$

$h F_{1}$

hE

h $E_{2}$

$h^{\prime} E_{s}$

$h^{\prime} \bar{E}_{25}$

$h^{m} F_{2}$

$\frac{h^{m} F_{1}}{h^{m} E}$

$\therefore$ -

2 a

$++1++1+$

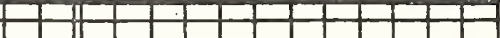




\section{IONOSPHERIG DATA RECORO}

Iermedec Is.

$\left(29.2^{05}, 377.90 \%\right)$

1943 a 184

$\log$

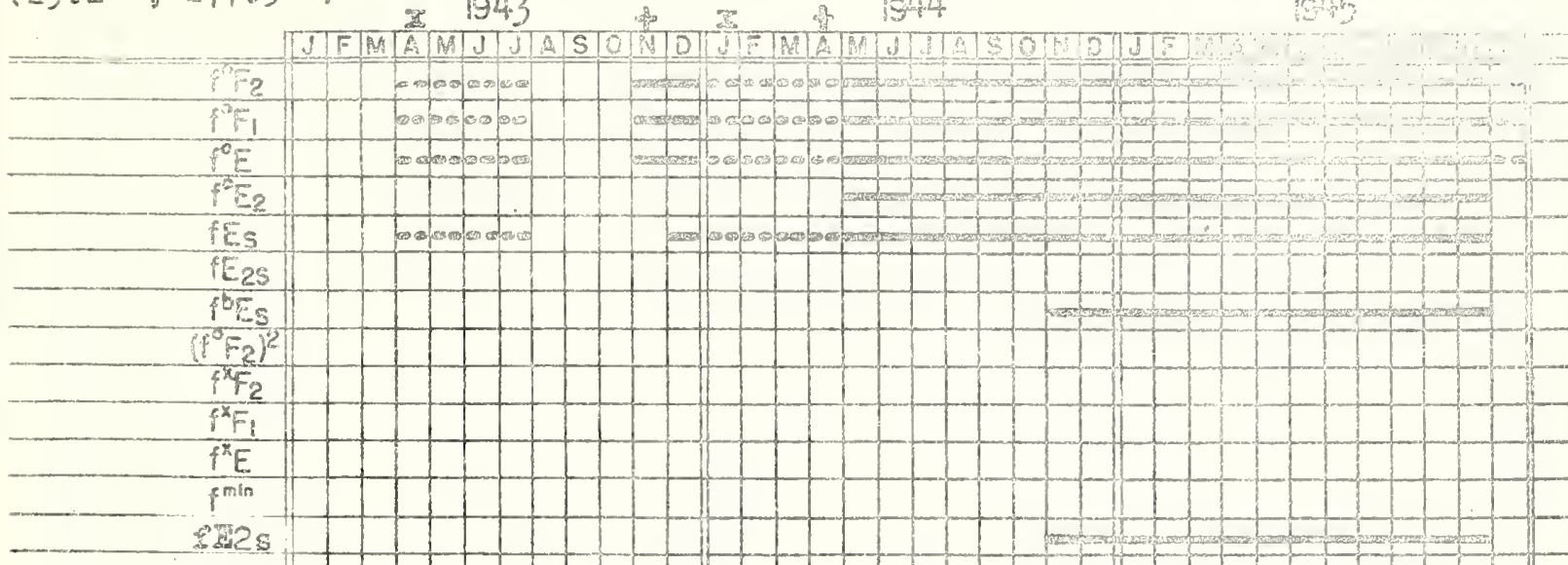

\begin{tabular}{cc}
\hline$F$ & muf \\
\hline$F$ & muf \\
\hline$F$ & muf \\
\hline$F$ & muf \\
\hline$F$ & muf \\
\hline$F$ & muf \\
\hline$E$ & muf \\
\hline \multicolumn{2}{|c|}{ M3500 } \\
\hline M 2000 \\
\hline$M$ \\
\hline$M$ \\
\hline$M$
\end{tabular}

* Fr P 3000

$* F_{2} 19000$

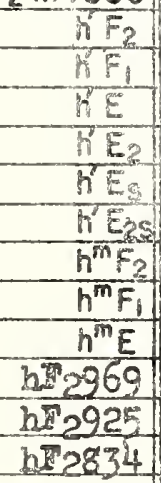

T

Absorption

* Calculated by IRPL.

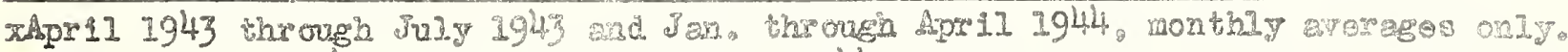

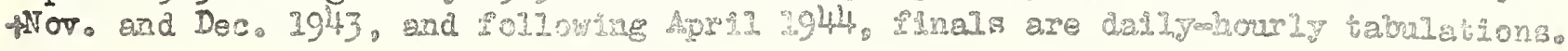



Tochel, Germars

$(4.70 \%, 11.40 \%)$
ICNOSPHERIC DATA RECOFO

194.4

1985 19

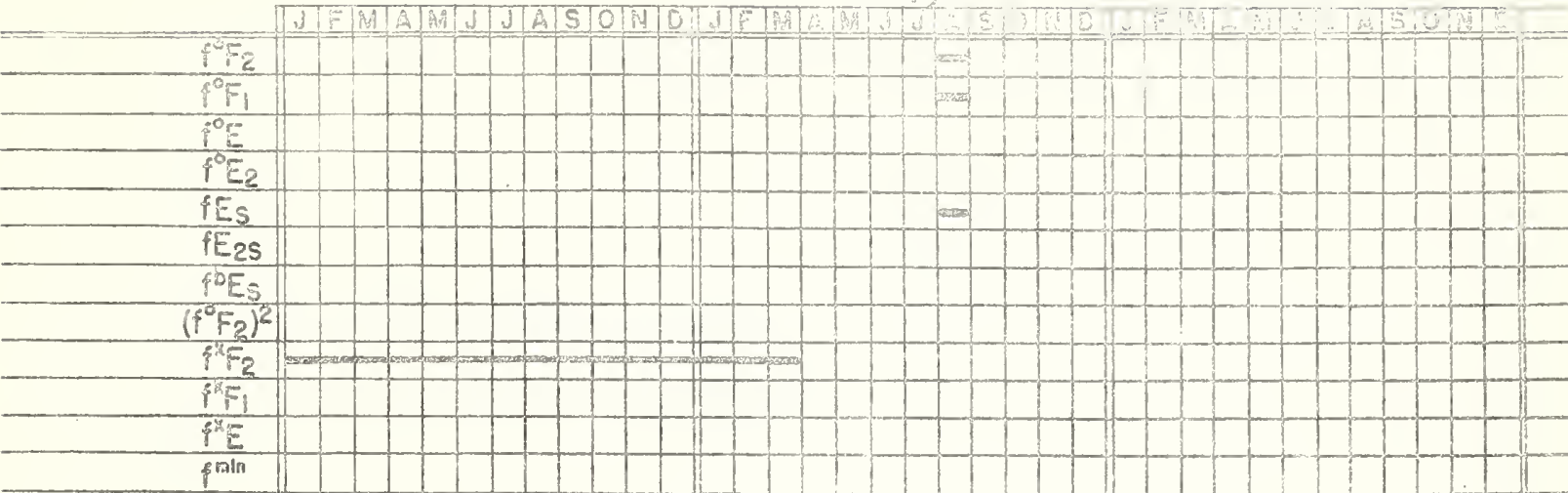

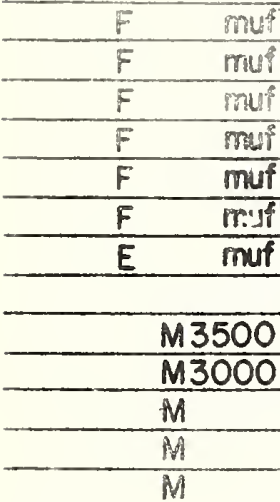

* Fe $M 3000$

* F $F_{2} M 4000$

$h_{2}$

h'

TंE

hE

$h^{\prime} E_{S}$

$h^{\prime} E_{2 s}$

$h^{m} F_{2}$

$h^{m} F_{1}$
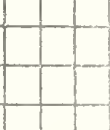

\section{he}



IONOSPHERIC DATA RECORD

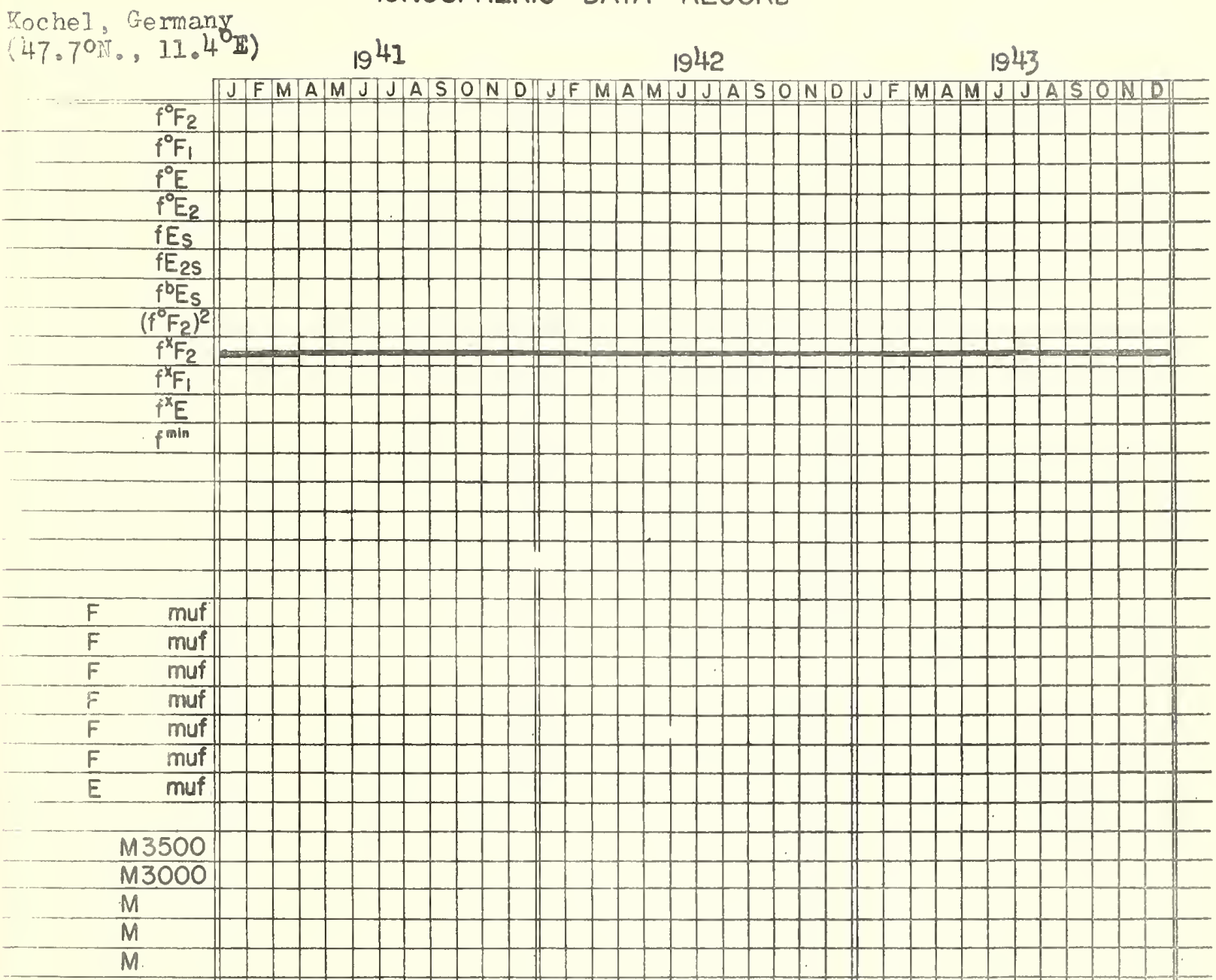

Kochel, Germany
$\left(47.7^{\text {ON. }}, 11.4 \mathrm{~W}\right.$

1941

1942

1943

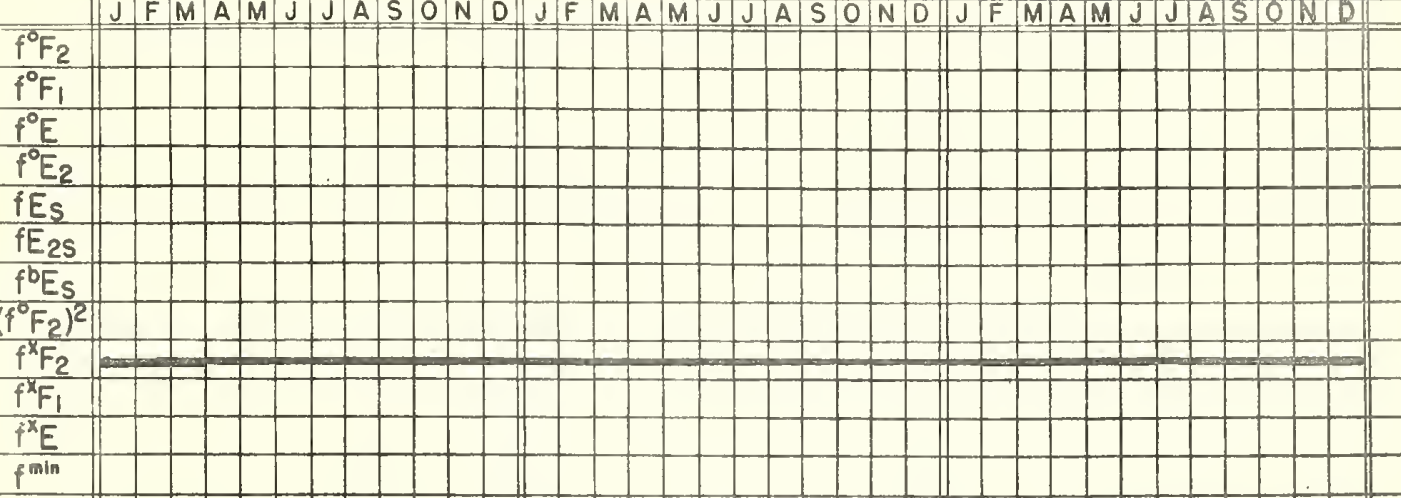

* F $\mathrm{F} 3000$

* $\mathrm{F}_{2} \mathrm{M} 4000$

$h^{\prime} F_{2}$

$h F_{1}$

hE

$h^{\prime} E_{2}$

$h^{\prime} E_{S}$

$h^{\prime} E_{\text {żs }}$

$h^{m} F_{2}$

$h^{m} F_{1}$

$h^{m} E$

Absorption

* Calculated by IRPL

Part of a U.S. Army Intelligence Report. Monthly Averages. 
Eochel, Gerrany

$\left(47.7^{\circ} \mathrm{N}, 11.40 \mathrm{~m}\right)$
IONOSPHEFUIC DATA RECORC

19381939

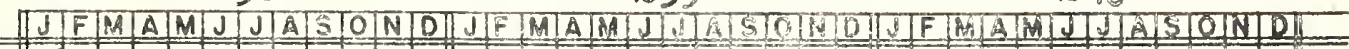

\begin{tabular}{cc}
\hline$F$ & muf \\
\hline$F$ & muf \\
\hline$F$ & musf \\
\hline$F$ & muif \\
\hline$F$ & muf \\
\hline$F$ & muf \\
\hline$E$ & muf \\
\hline \multicolumn{2}{c}{$M 3500$} \\
\hline \multicolumn{2}{c}{ M3000 } \\
\hline$M$ \\
\hline$M$ \\
\hline$M$
\end{tabular}

* $F_{2} M 3000$

* $\mathrm{F}_{2} \mathrm{M} 4000$

$h^{\prime} F_{2}$

$h^{\prime} F_{1}$

$h^{\prime} E$

$h^{\prime} E_{2}$

$h^{\prime} E_{s}$

$h^{7} E_{25}$

$h^{m} F_{2}$

$h^{m} E$

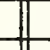

$+1$

$\sin$

ins

1)

$$
-
$$

II

$+\ldots+\ldots+2+1+1+$ 


$$
\text { • }
$$


ajalein $\Delta$ toll
$3.00 \mathrm{~N}, 168.0^{\circ} \mathrm{m}$

IONOSPHERIC DATA RECORD

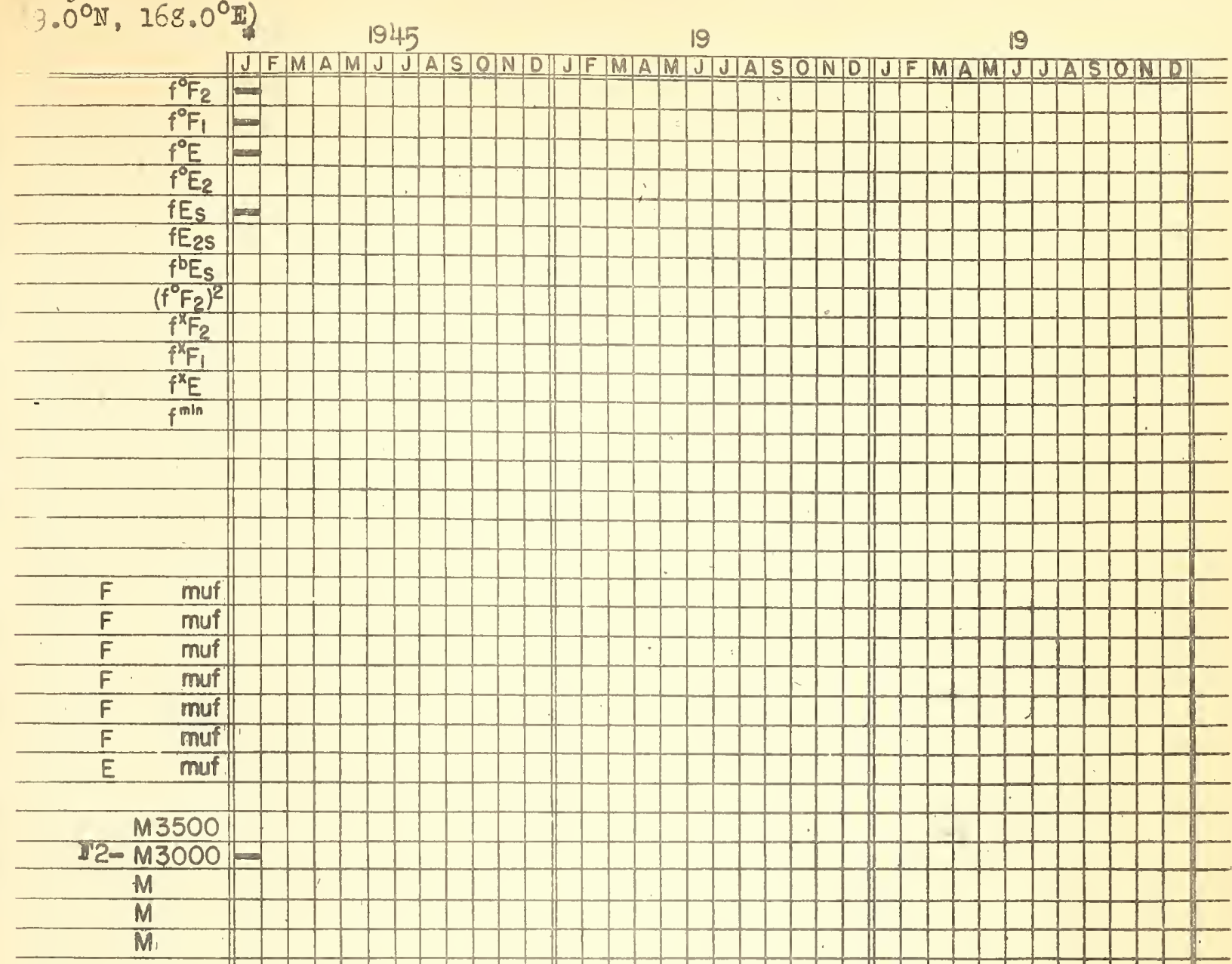

* $F_{2} M 3000$

* Fr M4000

\begin{tabular}{l|}
$h^{\prime} F_{2}$ \\
\hline$h^{\prime} F_{1}$ \\
$h^{\prime} E$ \\
\hline$h^{\prime} E_{2}$ \\
$h^{\prime} E_{S}$ \\
\hline$h^{\prime} E_{2 S}$ \\
$h^{m} F_{2}$ \\
\hline$h^{m} F_{1}$ \\
$h^{m} E$
\end{tabular}

Absorption

* Calculated by IRPL

* Irom 2000 January 10 to 2300 January 27. 1945, daily-hourly tabulations. 

Leningrad, U.S.S.R. IONOSPHERIC DATA RECORD

$\left(59.7^{\circ} \mathrm{NT}, 30.5^{\circ} \mathrm{E}\right)$

1945

19

19

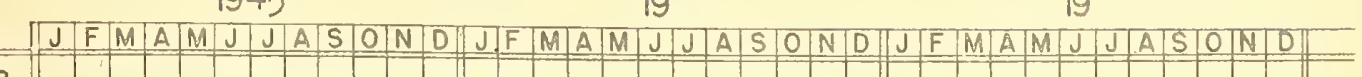

\begin{tabular}{|c|c|c|c|c|}
\hline${ }^{\circ} F_{1}$ & & & & \\
\hline$f^{\circ} E$ & & & & \\
\hline$f^{\circ} E_{2}$ & & & & \\
\hline$f E_{S}$ & & & & \\
\hline $\mathrm{fE}_{2 \mathrm{~S}}$ & & & & \\
\hline$f^{\mathrm{b}} E_{\mathrm{S}}$ & & & & \\
\hline$\left(f^{\circ} F_{2}\right)^{2}$ & & & & \\
\hline$\frac{x^{4} r_{2}}{f^{x} F_{i}}$ & & & & \\
\hline$f^{x} E$ & & & & \\
\hline$f^{\min }$ & & & & \\
\hline
\end{tabular}

\begin{tabular}{cc}
\hline$F$ & muf \\
\hline$F$ & muf \\
\hline$F$ & muf \\
\hline$F$ & muf \\
\hline$F$ & muf \\
\hline$F$ & muf \\
\hline$E$ & muf \\
\hline$M 3500$ \\
\hline$M 3000$ \\
\hline$M$ \\
\hline$M$ \\
\hline$M$ \\
\hline$M$
\end{tabular}

* $\mathrm{F}_{2} \mathrm{M} 3000$

* $\mathrm{F}_{2} \mathrm{M} 4000$

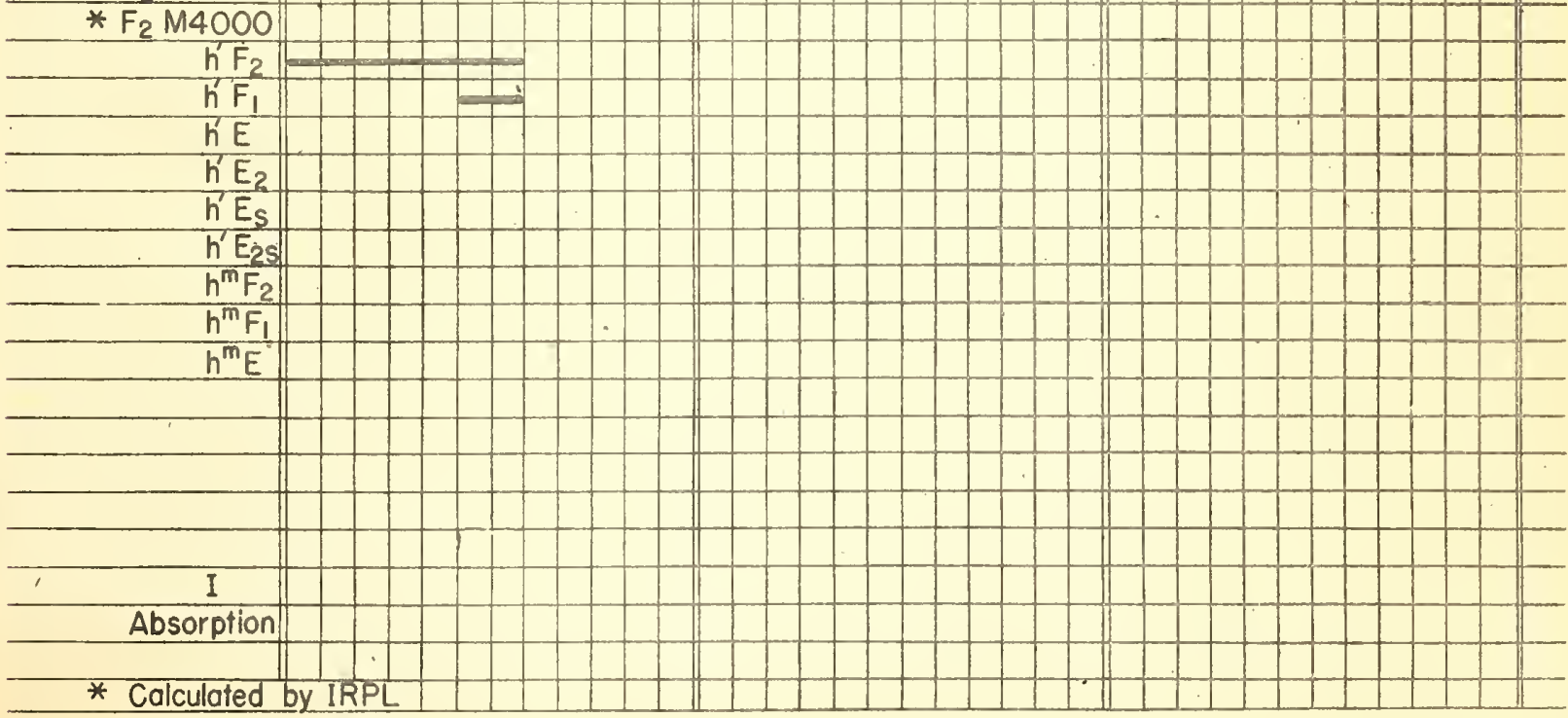

Monthly averages. 
. 
Leyte, Philippine Is。

\section{IONOSPHEPIC DATA RECORD}

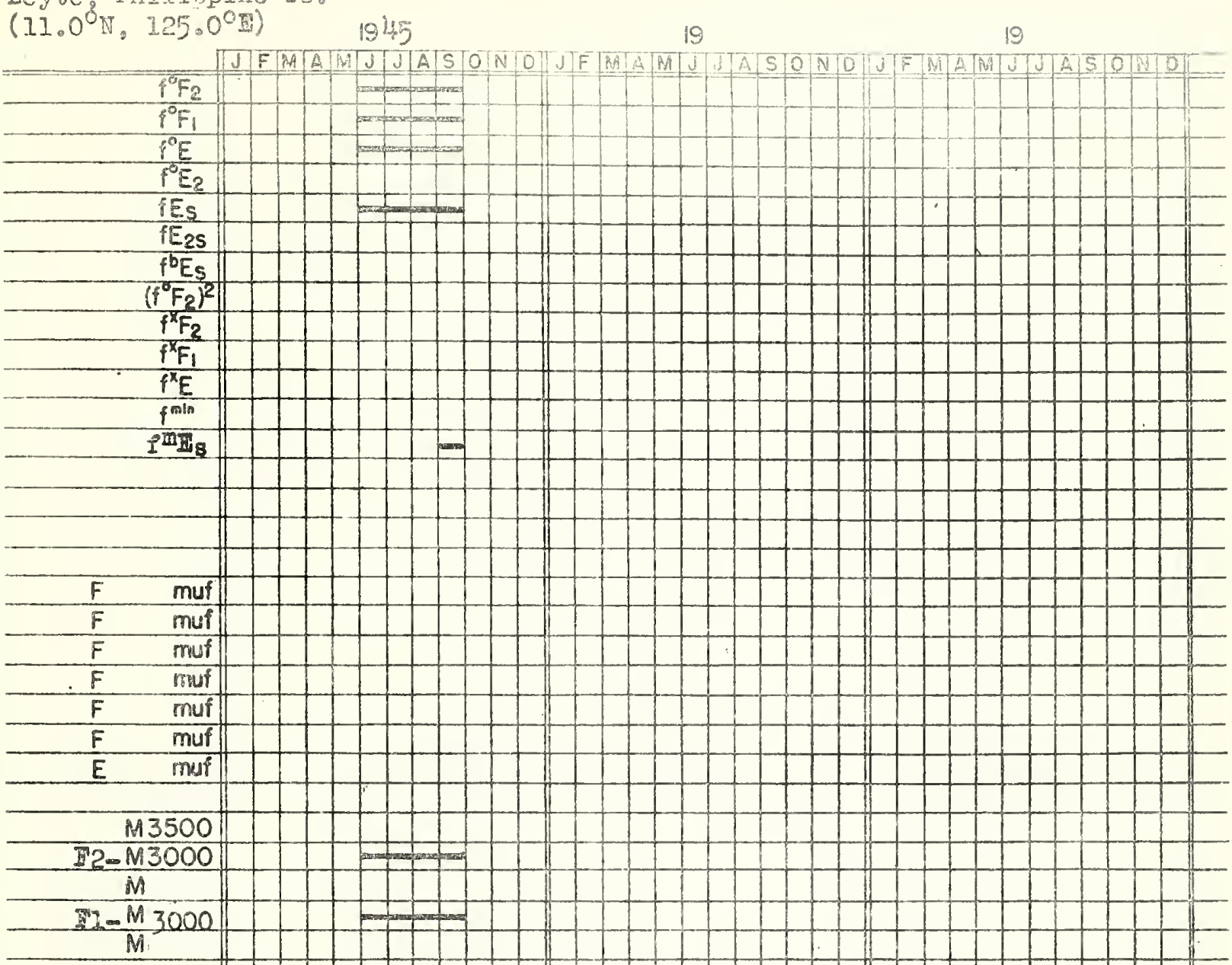

* $\mathrm{F}_{2} \mathrm{M} 3000$

* $F_{2} M 4000$

$h^{\prime} F_{2}$

h'E

$h^{\prime} E_{2}$

$h^{\prime} E_{S}$

$h^{\prime} E_{2}$

$h^{m} F_{2}$

$i^{m} F_{1}$

$h^{m} E$

(1)

I 
Majras, India

IONOSPHERIC DATA RECORD

$\left(13.0^{\circ}, 80.2^{\circ}\right)$
1945

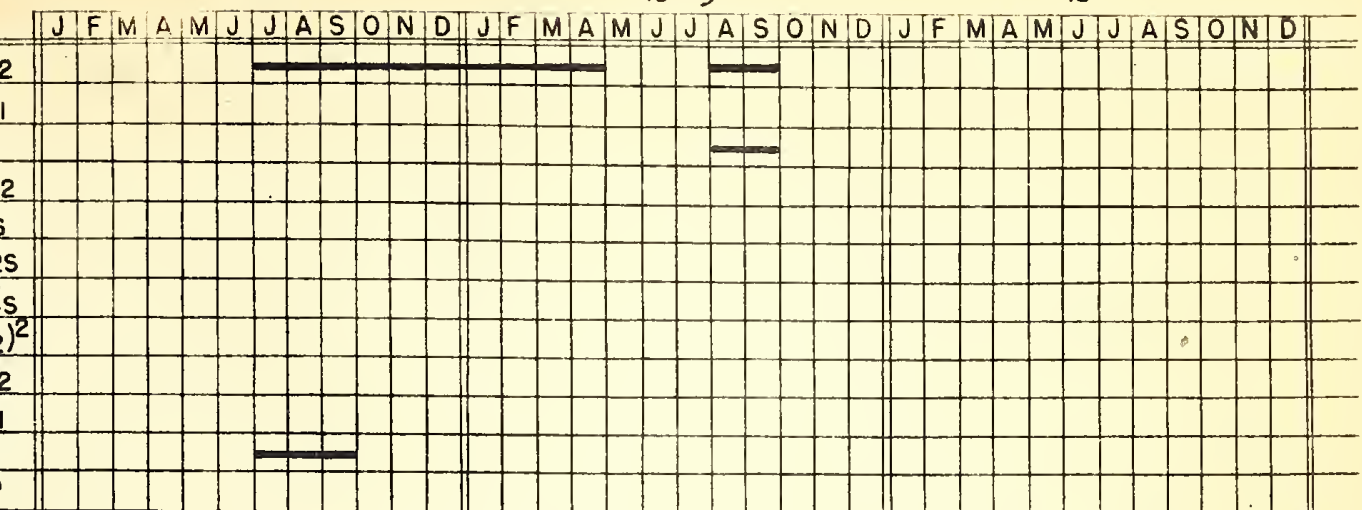

\begin{tabular}{cc|}
\hline$F$ & muf \\
\hline$F$ & muf \\
\hline$F$ & muf \\
\hline$F$ & muf \\
\hline$F$ & muf \\
\hline$F$ & muf \\
\hline$E$ & muf
\end{tabular}

F2- $M 3500$

F2 $M 3000$

F2 $M 2500$

F2.M 2000

F2-M 1500

$\$ 2-M 1000$

F2-M 500

* F $\mathrm{M} 3000$

* $F_{2} M 4000$

$h^{\prime} F_{2}$

h' $F_{1}$

h'E

$h^{\prime} E_{2}$

$h^{\prime} E_{S}$

$h^{\prime} E_{2 s}$

$h^{m} F_{2}$

$h^{m} F_{1}$

$+$

$+1+1$ 
Mau1, Hawaii

$\left(20.8^{\circ} \mathrm{N}, 156.5^{\circ} \mathrm{W}\right)$

IONOSPHERIC DATA RECORD

1944
19

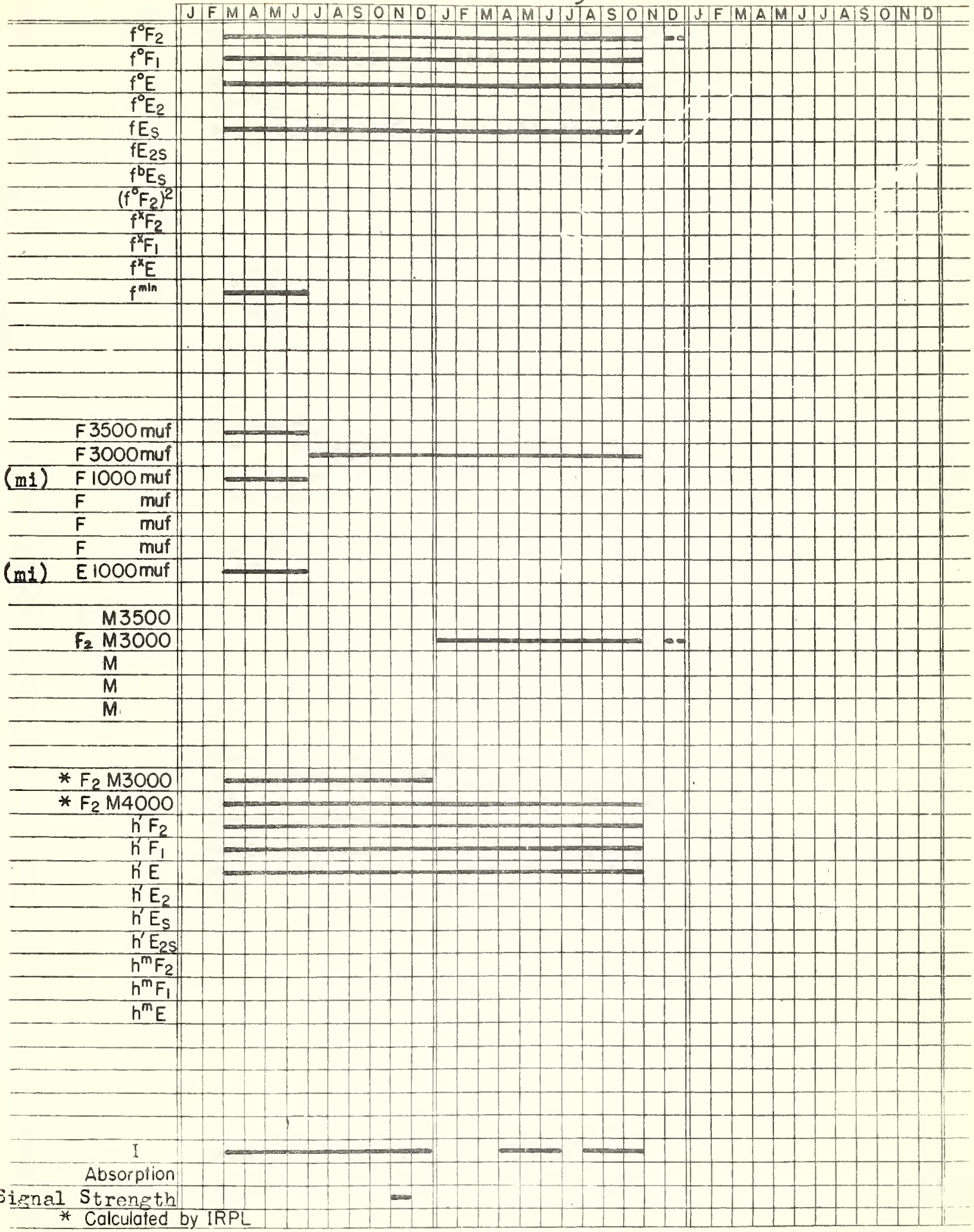


Yosece T.S.S.R.

ONOSPHERUC DATA RECORO

$\left\{55.3^{0} \mathrm{iT}, 37.50 \mathrm{R}\right)$

$19: 4$

315

19

F

$f^{3} E$

$f^{\circ} E_{2}$

fEs.

TERS

fos

$\frac{1 f^{3} F_{2} t^{2}}{f^{2} F_{2}}$

$f^{x} F_{1}$

$-1+1+1-1+1$

$=1-1$

$\frac{f^{2} E}{f^{m}}$

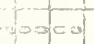

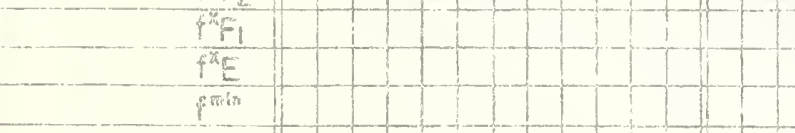

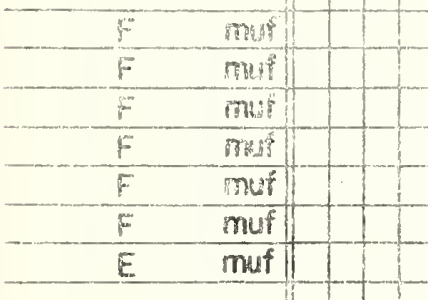

M3500

M MOOOO

M

$M$

* Fe M 3000

* Fq MaOOO

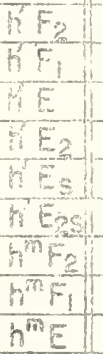

Absopption

* Calculcred by TP.

Monthly avenages only. 

Oslo, Morwey

(53.30\%, $\left.71.0^{\circ} \mathrm{z}\right)$

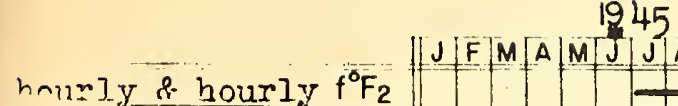

hourly \& hourly $f^{\circ} F_{1}$

'lourly \& hourly $f^{\circ} \mathrm{E}$

hourly \& hourly $\frac{f E_{S}}{f E_{2 S}}$

fE 25

hourl $\frac{\left(f^{\circ} \mathrm{F}_{2}\right)^{2}}{\mathrm{f}^{\mathrm{f}} \mathrm{F} \mathrm{F}_{2}}$

$f^{x} F_{1}$

$f^{x} E$

\begin{tabular}{|ll|}
\hline$F$ & muf \\
\hline$F$ & muf \\
\hline$F$ & muf \\
\hline$F$ & muf \\
\hline$F$ & muf \\
\hline$F$ & muf \\
\hline$E$ & muf \\
\hline
\end{tabular}

$\frac{M 3500}{\frac{M 3000}{M}} \frac{\frac{M}{M}}{}$

* $\mathrm{F}_{2} \mathrm{M} 3000$

* $\mathrm{F}_{2} \mathrm{M} 4000$

$h^{\prime} F_{2}$

$h^{\prime} F_{1}$

T'E

$h^{\prime} E_{2}$

$h^{\prime} E_{S}$

$h^{\prime} E_{25}$

$h^{m} F_{2}$

$\frac{h^{m} F_{1}}{h^{m} E}$

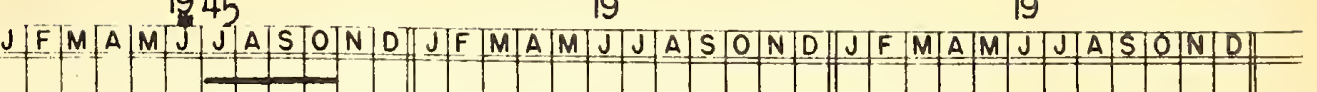





\section{IONOSPHERIC DATA RECORD}

Oslo, Norway

$\left(59.9^{\circ} \mathrm{N}, 11.0 \mathrm{O}\right)$

1942

1943

1944

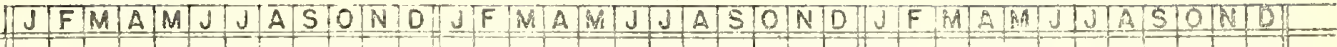

$\frac{f^{\infty} F_{2}}{f^{3} F_{1}}+:+$

$f^{\circ} E_{2}$

fEs

FE:S

${ }^{D} E_{S}$

$\left(8^{\circ} \mathrm{F}_{2}\right)^{2}$

$f^{\mathrm{x}} F_{2}$

$f^{\mathrm{N}} \mathrm{F}_{1}$

$f^{\mathrm{R} E}$

$f^{\text {nin }}$

$\begin{array}{cc}F & \text { must } \\ F & \text { muf } \\ F & \text { muf } \\ F & \text { mut } \\ F & \text { mest } \\ F & \text { muf } \\ E & \text { mof }\end{array}$

$M 3500$

$M 3000$

$M$

$M$

M

F F M3000

* $F_{2} M 4000$

$h^{\prime} F_{2}$

$h^{\prime} F_{1}$

WE

HE

तंEs

hers

$h^{\mathrm{m} F}$

$h^{m} F_{1}$

$h^{6} \mathrm{E}$

\section{I}

Absorotion:

\% Colculoted by 12 LL

Sert. $2945,16 \mathrm{~m} 30$ ondy"

Oct. 1943, 36-3I oniy.

TOF。 $1943 \quad 1002515$

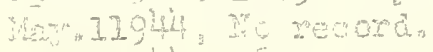

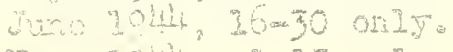

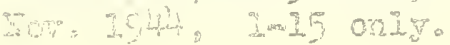



Ot,tawa, Canada
$\left(4+5.5^{\circ \mathrm{N}}, 75.8^{\circ} \mathrm{W}\right)$

IONOSPHERIC DATA RECORD

1945

19

19

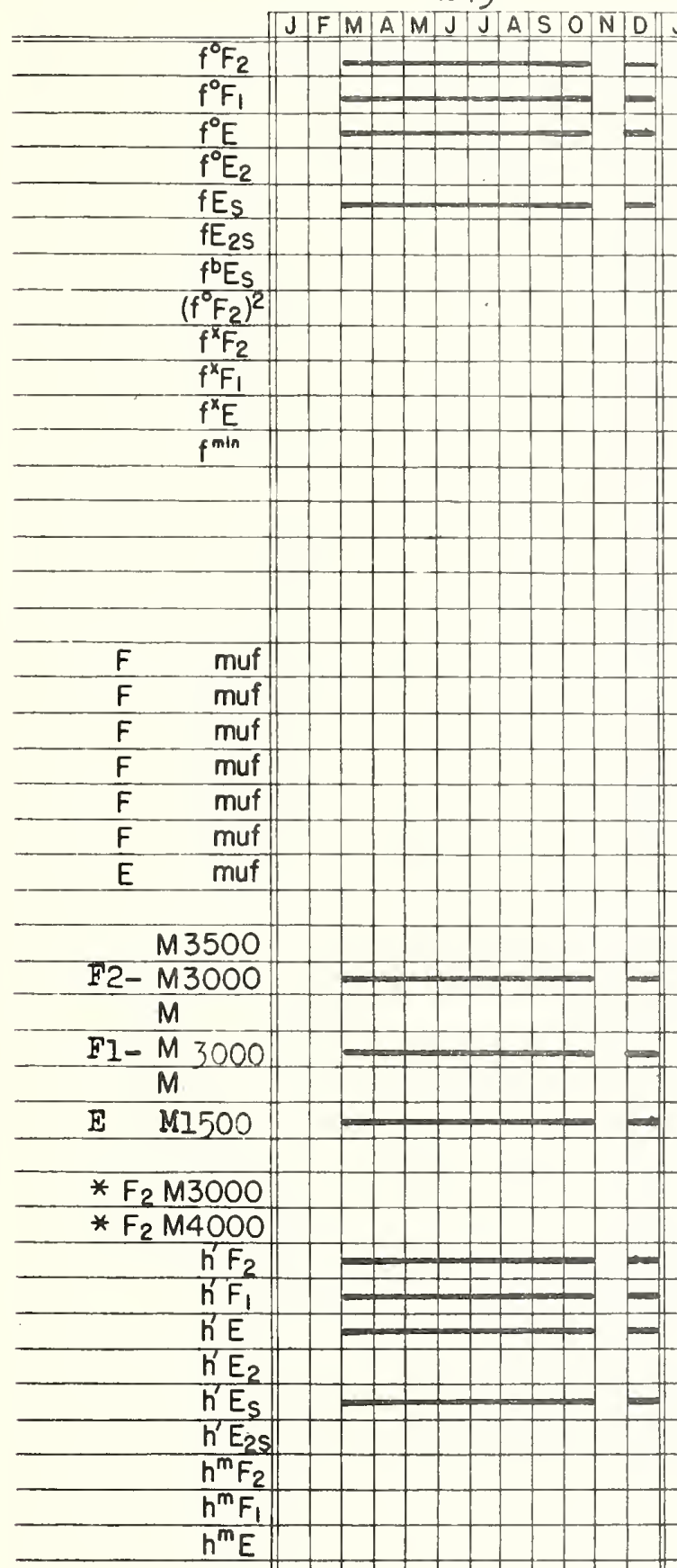

I

Absorption 



Ottawn, Csmada IONOSPHERIC DATA RECORD

$(45.50 \%, 75.90 \%) \quad 19 ! 2$

1943

$194 \cdot 4$

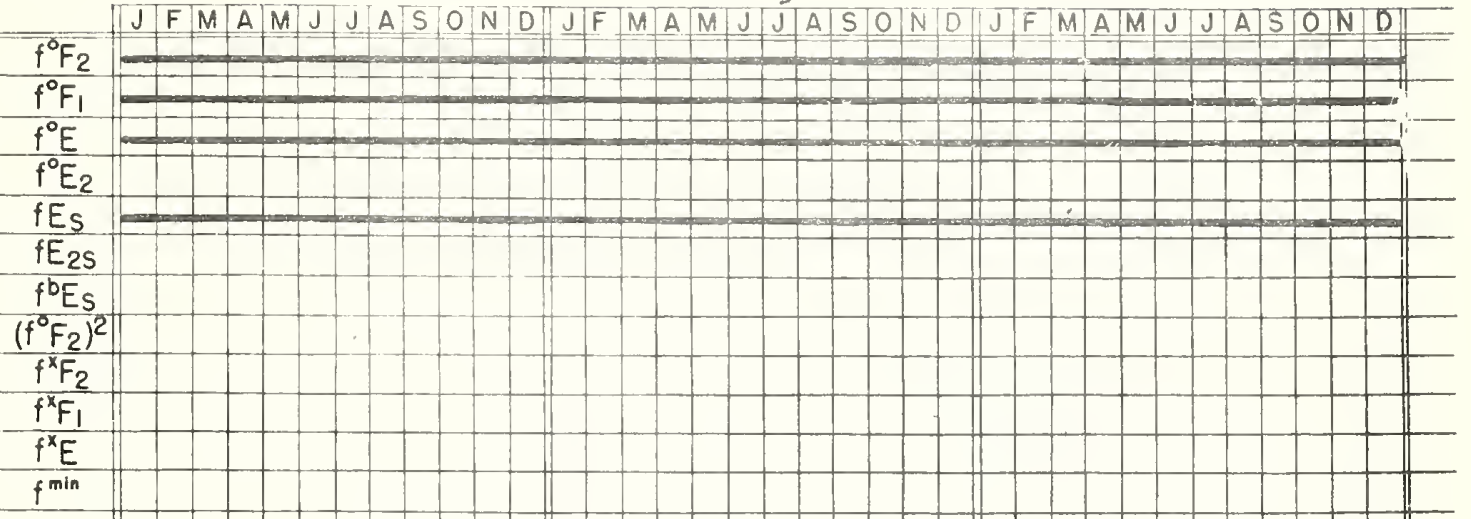

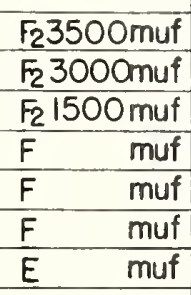

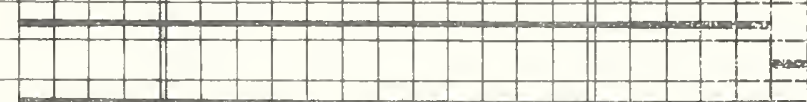

$M 3500$

F2. M 3000

Fl-M 3000 $M$

M

* $F_{2} M 3000$

* $\mathrm{F}_{2} \mathrm{M} 4000$

$h^{\prime} F_{2}$

h'F

$h^{\prime} E_{2}$

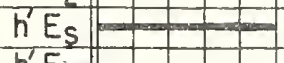

$4-1212$

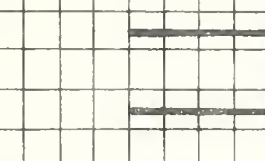

$\frac{h^{m} F_{2}}{h^{m} F_{1}}$

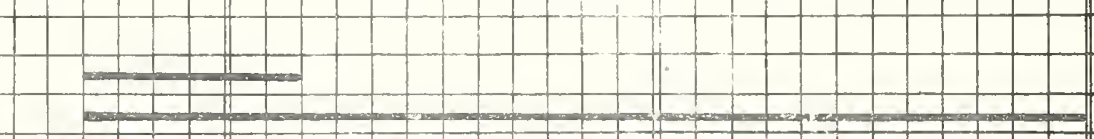

$-1-1$

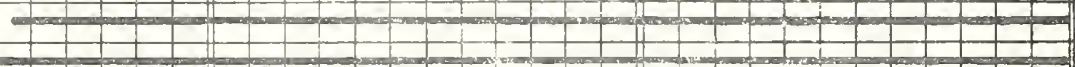

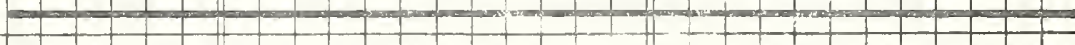

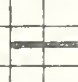

E

Arrora

$-1+20$

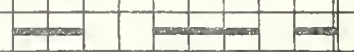



Palau Is.

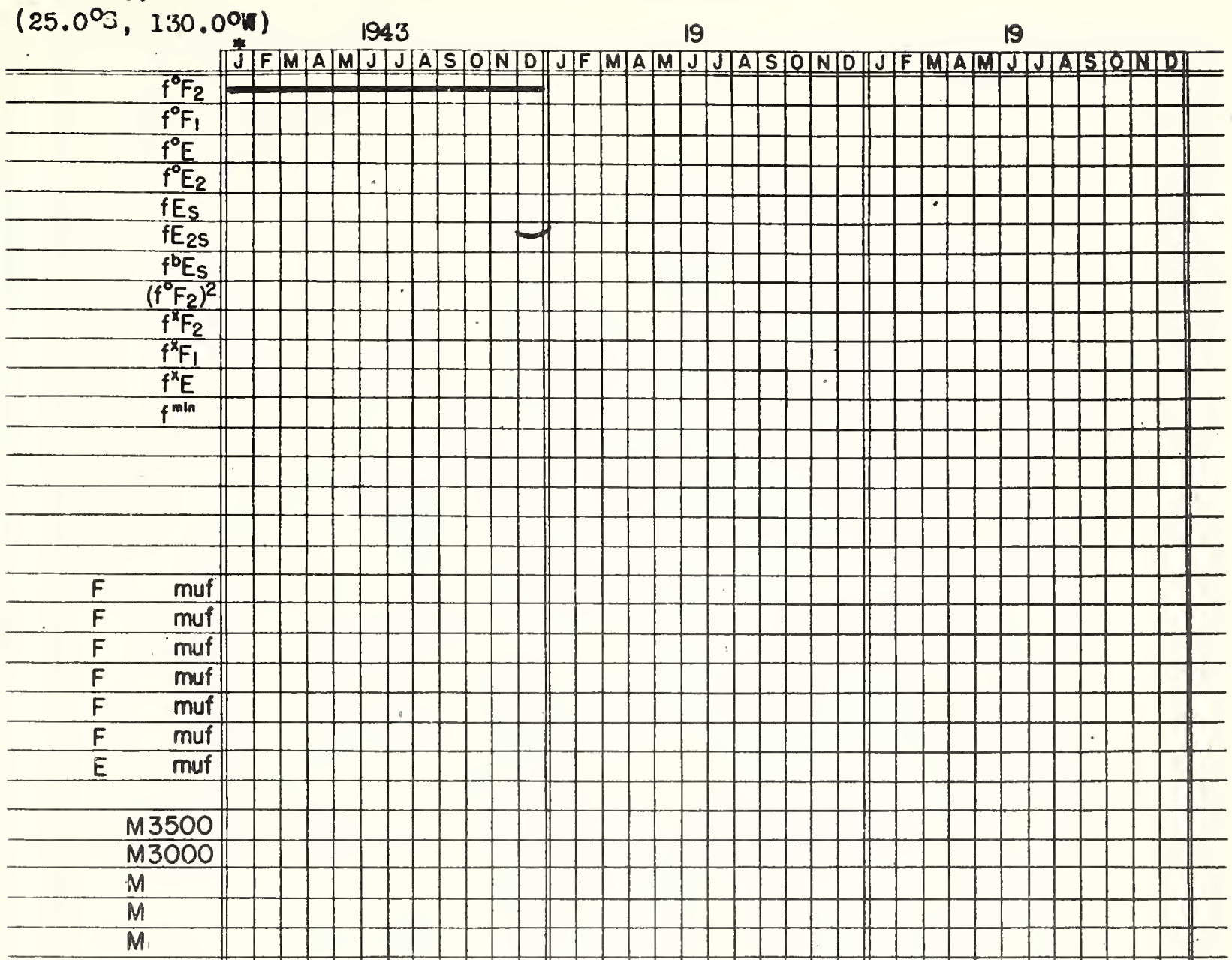

* F 2 M3000

* Fr M4000

$h^{\prime} F_{2}$

$h^{\prime} F_{1}$

h'E

$h^{\prime} E_{2}$

$h^{\prime} E_{S}$

$h^{\prime} E_{2 s}$

$h^{m} F_{2}$

$h^{m} F_{1}$

\section{3}


Feshawar, India

IONOSPHERIC DATA RECORD

$\left(34.00 \mathrm{~N}, 71.5^{\circ}\right)$

1945

19

19

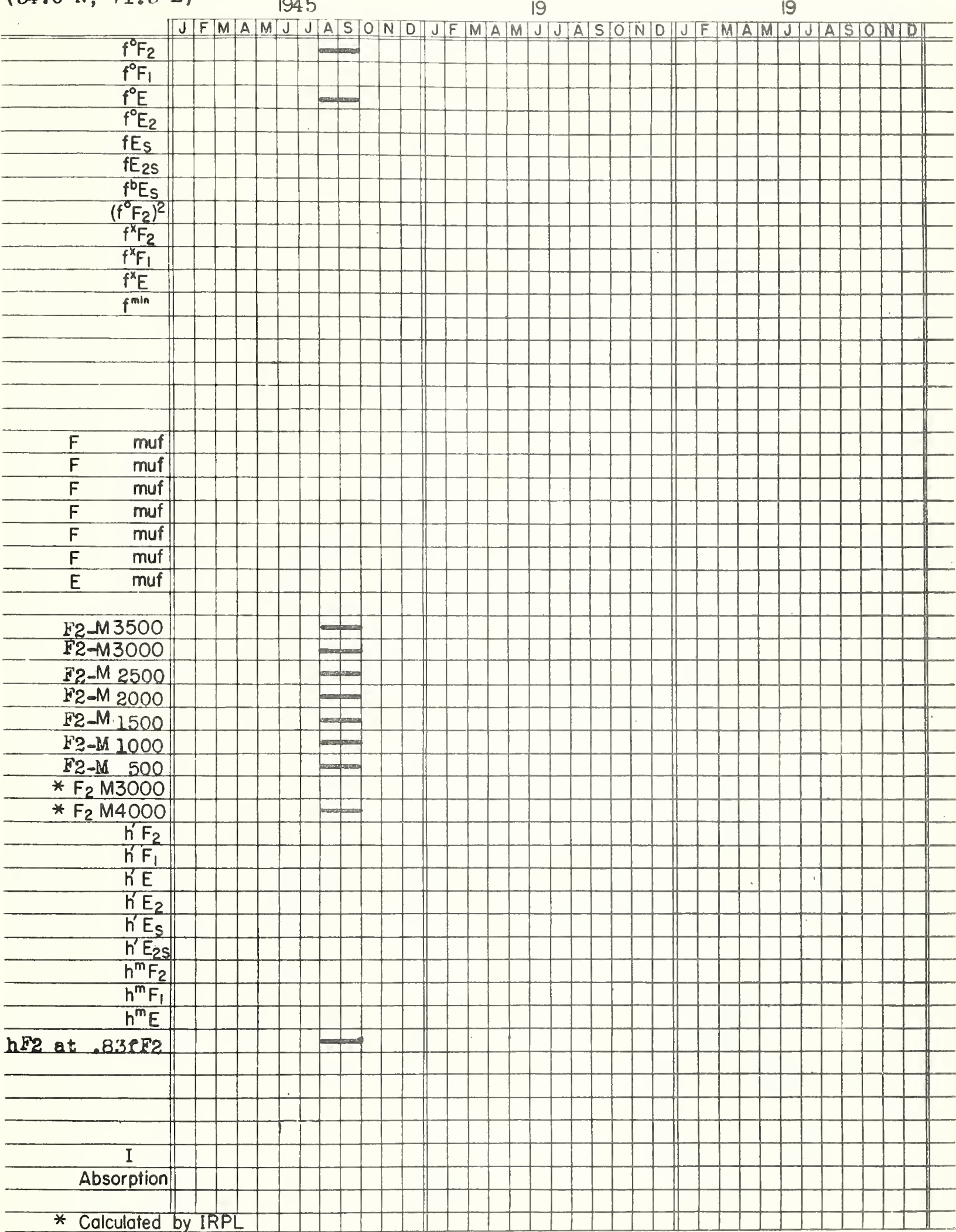



Pitcaira I.

IONOSPHERIC DATA RECORD

(25.005, 130.00\%)

1944

1945

19

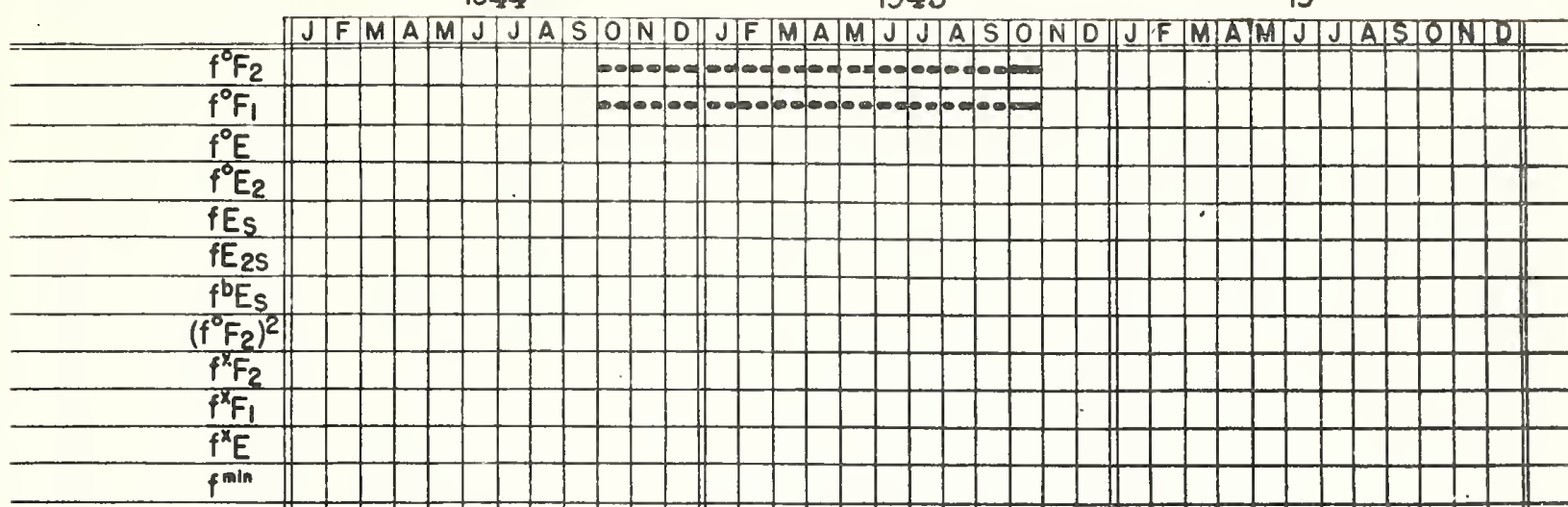

\begin{tabular}{cc|}
\hline$F$ & muf \\
\hline$F$ & muf \\
\hline$F$ & muf \\
\hline$F$ & muf \\
\hline$F$ & muf \\
\hline$F$ & muf \\
\hline$E$ & muf \\
\hline \multicolumn{2}{c}{$M 3500$} \\
\hline \multicolumn{2}{c}{$2-M 3000$} \\
\hline$M$ \\
\hline$M$ \\
\hline \multicolumn{2}{c}{$M$} \\
\hline
\end{tabular}

* $F_{2} M 3000$

* $F_{2} M 4000$

\begin{tabular}{l|}
$h^{\prime} F_{2}$ \\
$h^{\prime} F_{1}$ \\
$h^{\prime} E$ \\
$h^{\prime} E_{2}$ \\
$h^{\prime} E_{s}$ \\
$h^{\prime} E_{2}$ \\
$h^{m} F_{2}$ \\
$h^{m} F_{1}$ \\
$h^{m} E$ \\
\hline
\end{tabular}

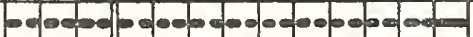

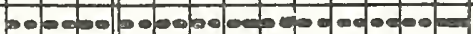

I

Absorption

* Calculated by IRPL

To May 31, 1945, monthly averages.

Since June 1, 1945, monthly eødiens.

Daily-hourly tabulations to come. 

Prince Rupert, Canade

$\left(54.30 \mathrm{~N}, 130.3^{\circ} \mathrm{W}\right)$

1945
19
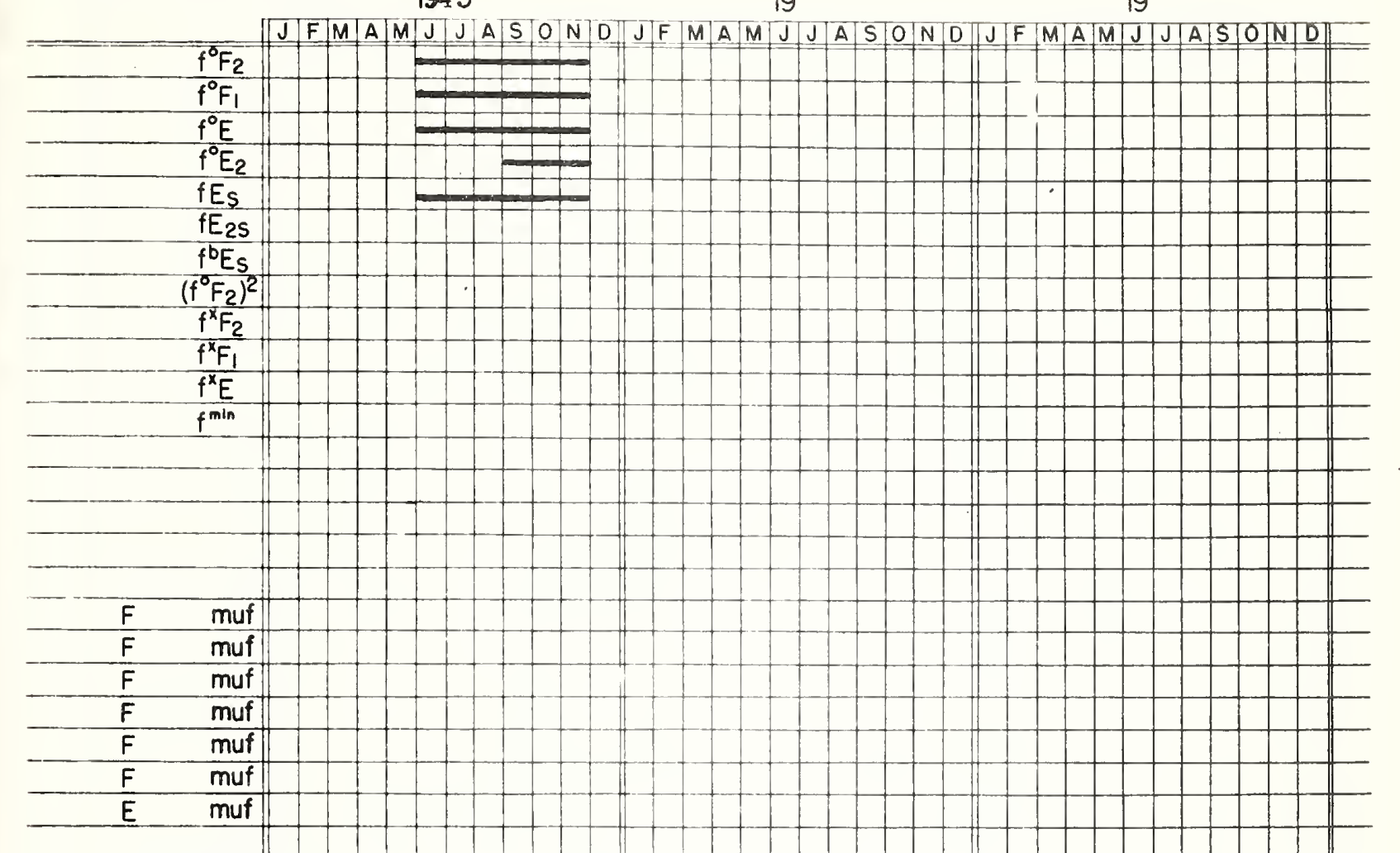

F2-M3000

$\frac{M}{M}$

I M 1500 .

* $F_{2} M 3000$

* $\mathrm{F}_{2} \mathrm{M} 4000$

$h^{\prime} F_{2}$

$h^{\prime} \mathrm{F}_{1}$

$h^{\prime} E$

$h^{\prime} E_{2}$

$h^{\prime} E_{S}$

$h^{\prime} E_{2 s}$

$h^{m} F_{2}$

$h^{m} F_{1}$

IONOSPHERIC DATA RECORE 

Rarotongra I.

(21.4 ${ }^{\circ}, 159.6^{\circ}$ W)

\section{5}

19
19

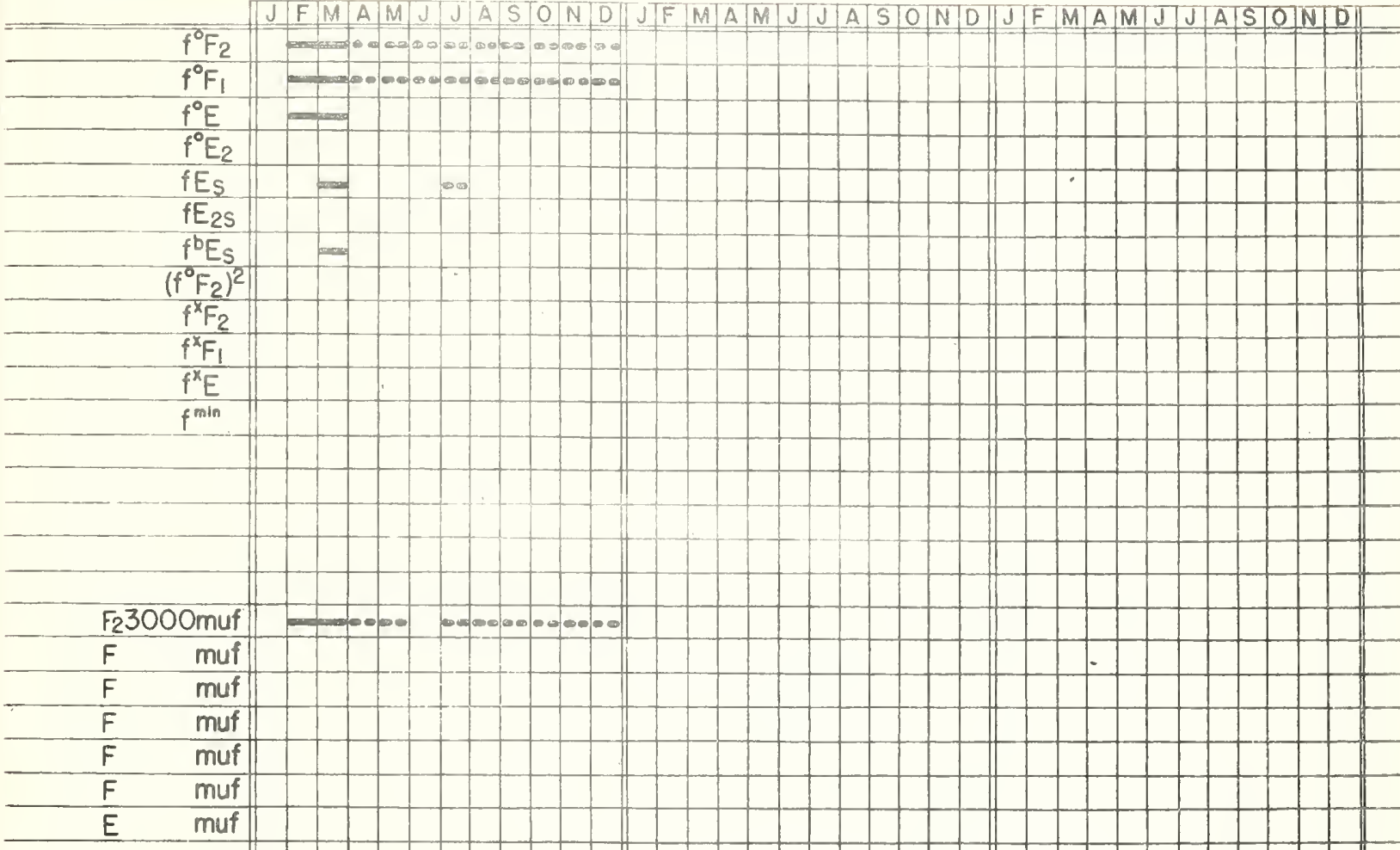

\begin{tabular}{c}
\hline M3500 \\
\hline$M 3000$ \\
\hline$M$ \\
\hline$M$
\end{tabular}

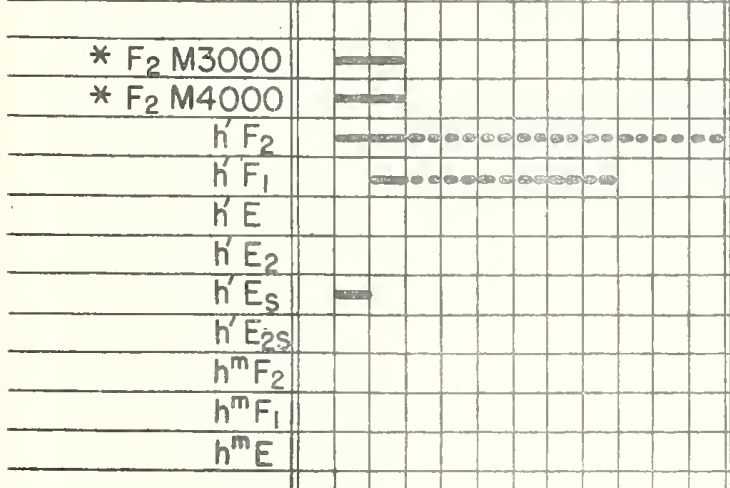

Absorption

* Calculated by IRPL

To May 31, 1945, monthly avertaes.

From June 1, 1945, monthly medians.

Daily-hourly tebulations to come. 

Rerizjavik, Iceland

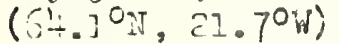

194 it 1945

19

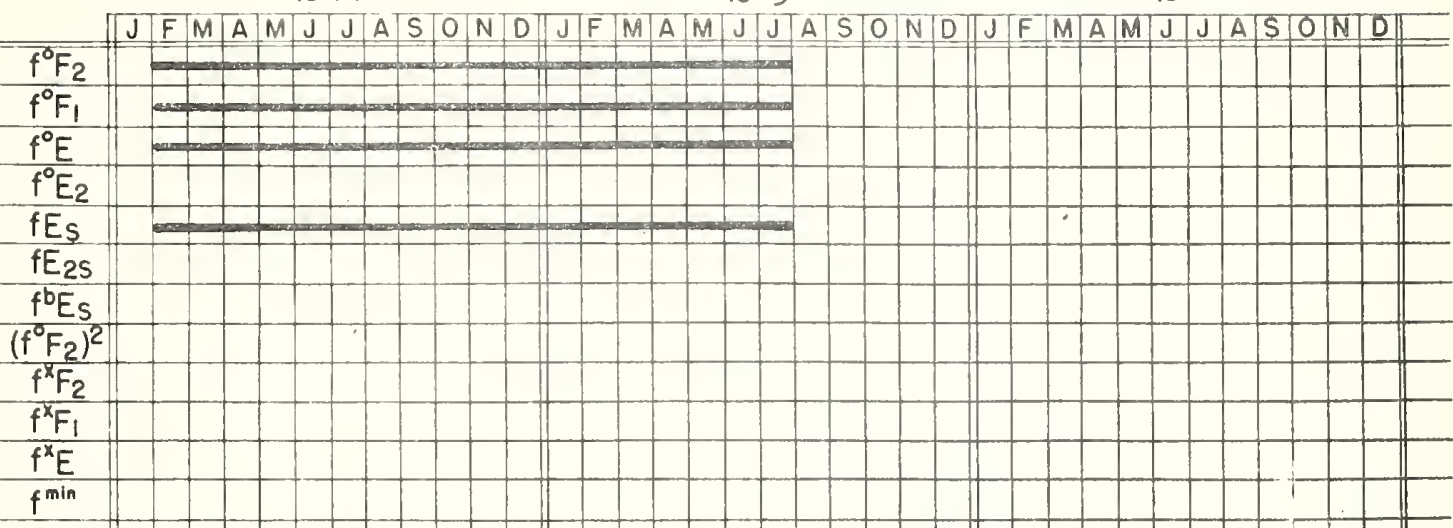

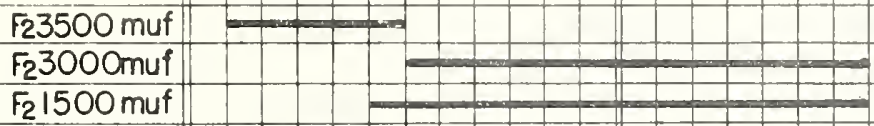

(ini) $F_{2} 1000$ muf

$F$ muf

$F$ muf

E muf

M3500

M3000

$M$

$M$

* $\mathrm{F}_{2} \mathrm{M} 3000 \mathrm{~F}-\mathrm{W}$

$h^{\prime} F_{2}$

h' $F_{1}$

h'E

$h^{\prime} E_{2}$

$h^{\prime} E_{S}$

$h^{\prime} E_{2 S}$

$h^{m} F_{2}$

$h^{m} F_{1}$

$h^{m} E$

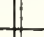

\begin{abstract}
$+$
\end{abstract}
Blackoints

Portial B? ackouts

Absorption

* Calculated by IRPL

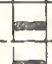

1 1

1 and 

3. Jom! Werfondan IONOSPHERIC DATA RECORD

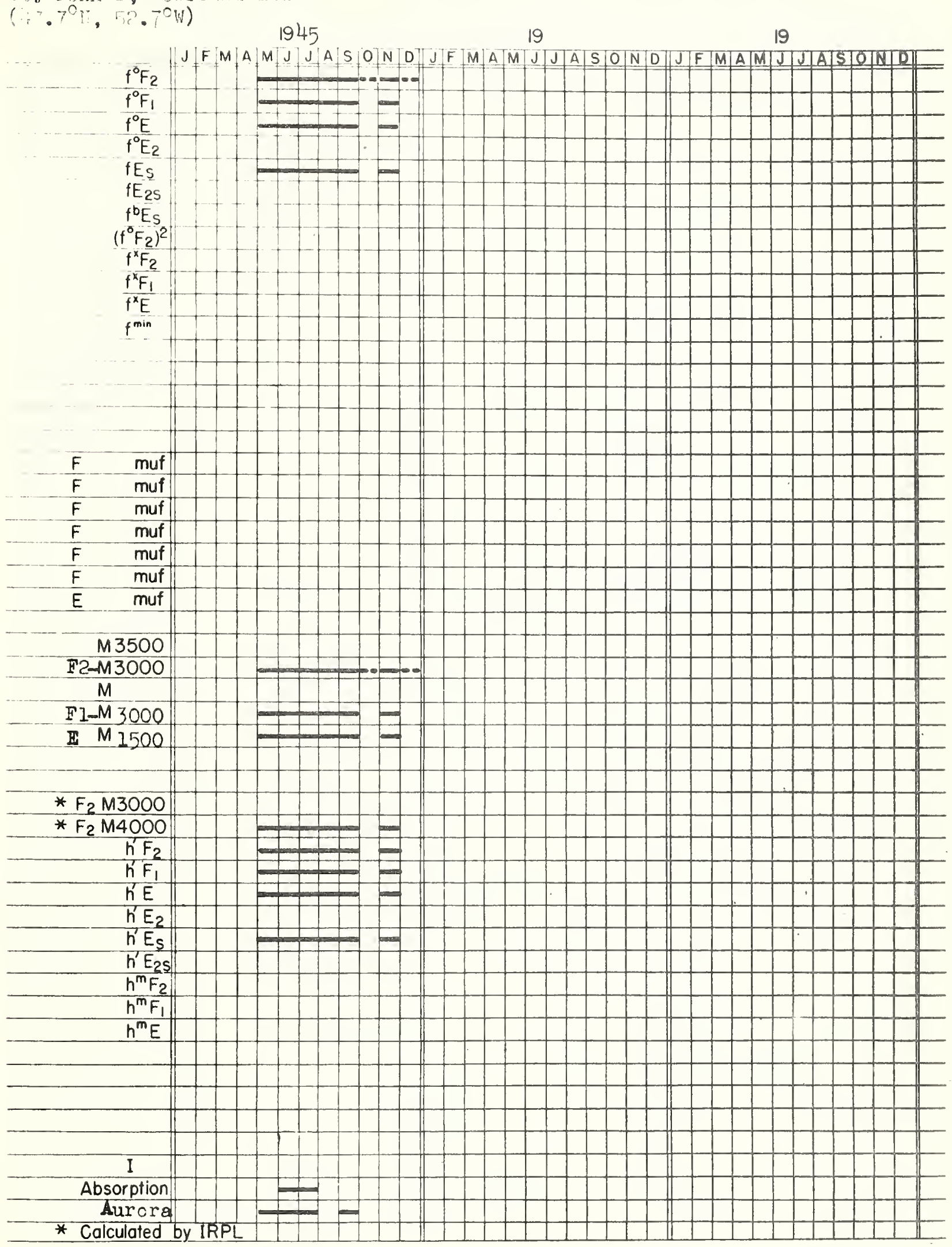


Sakhalin I.

IONOSPHERIC DATA RECORD

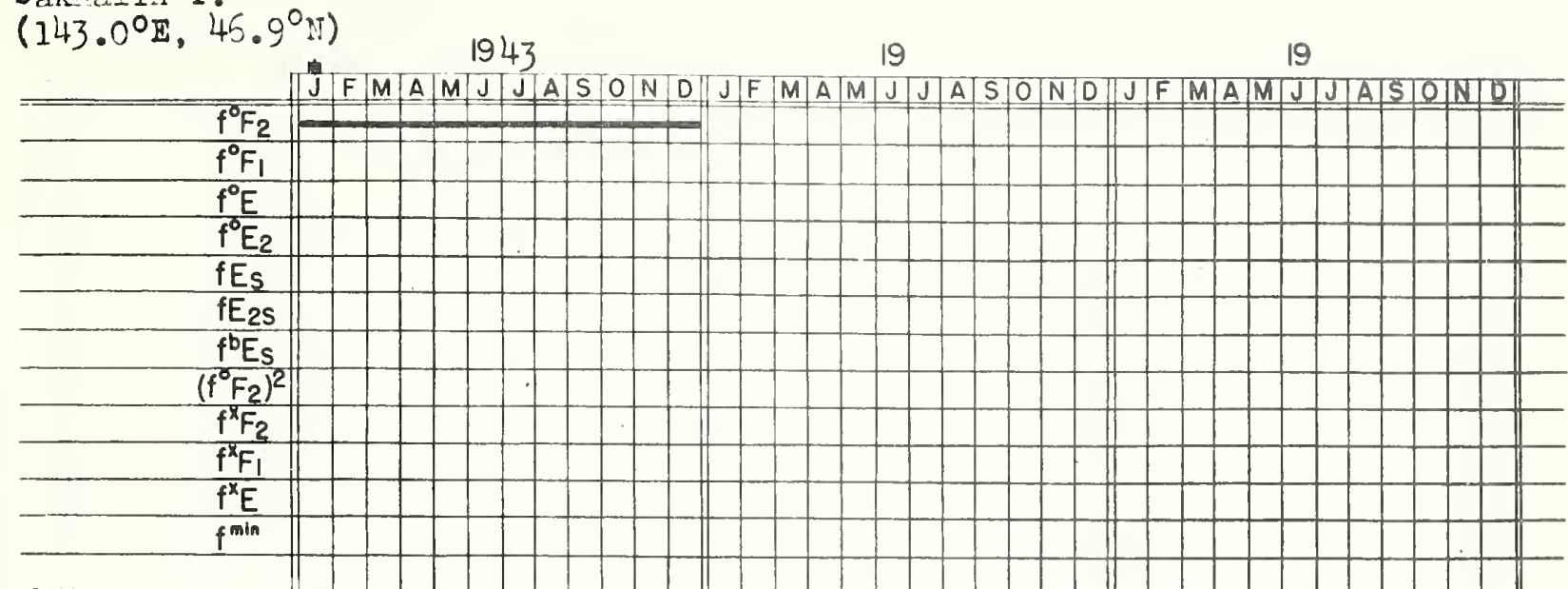

\begin{tabular}{ll||}
$F$ & muf \\
$F$ & muf \\
$F$ & muf \\
$F$ & muf \\
$F$ & muf \\
$F$ & muf \\
\hline$E$ & muf
\end{tabular}

$M 3500$

M3000

M

* $\mathrm{F}_{2} \mathrm{M} 3000$

* $\mathrm{F}_{2} \mathrm{M} 4000$

$h^{\prime} F_{2}$

$h^{\prime} F_{1}$

$h^{\prime} \mathrm{E}$

$h^{\prime} E_{2}$

$h^{\prime} E_{S}$

$h^{\prime} E_{2 s}$

$h^{m} F_{2}$

$h^{m} F_{1}$

$h^{m} E$

Absorption

* Calculated by IRPL

Graphs only. 
San Francisco, California ONOSPHERIC DATA RECORD

$\left(37.4^{\circ} \mathrm{N}, 122.2^{0} \mathrm{~m}\right)$

1945

19

19

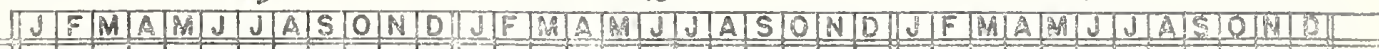

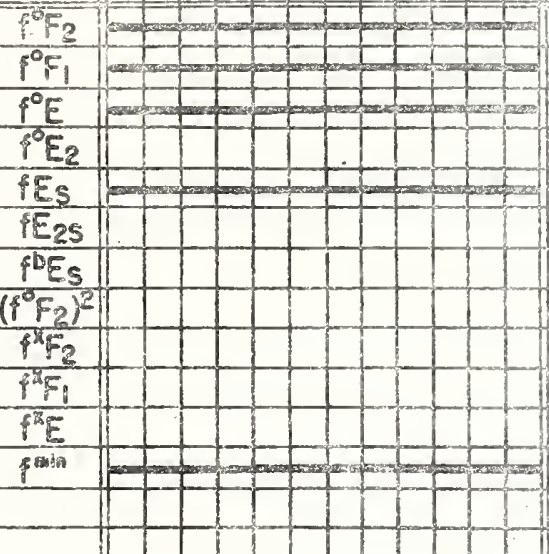

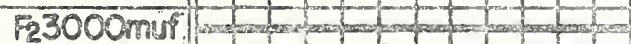

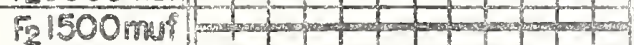

F mplotis

F $3000 \mathrm{mof}$ i

F muf

$\varepsilon$

M 3500

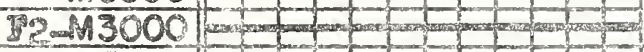

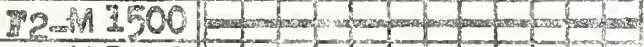

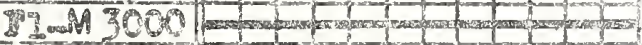
I

* FaM 3000

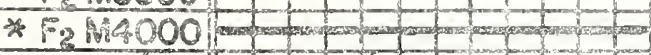

$h^{2} F_{2}$.

h.

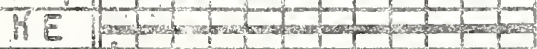

$\frac{1 E_{2}}{n E_{s}}$

WE

$h^{m} F_{2}$

$h^{m} F_{1}$

-

$+1$ 

San Francisco, California IONOSPHERIC DATA RECORD

$\left(37.4{ }^{\circ} \mathrm{N}, 122.2^{\circ} \mathrm{W}\right)$
1943

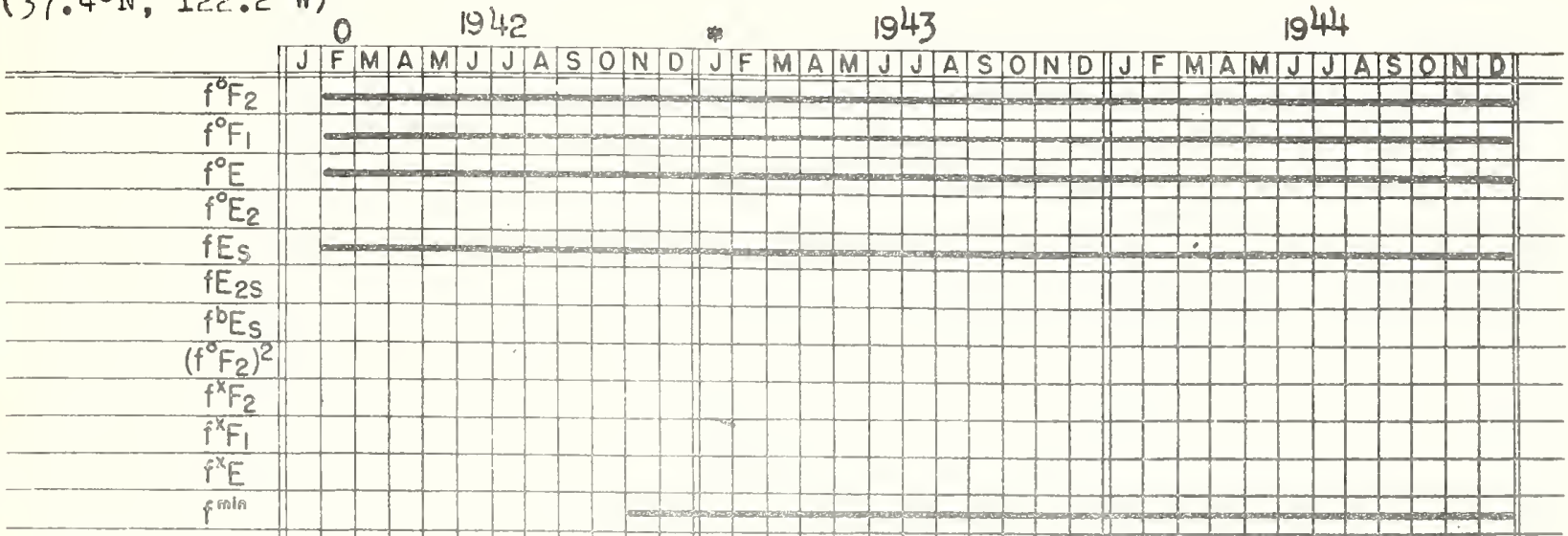

1944

(mi) $\sqrt{2} 3500$ muf hrsdmi) Fe 3500muf F21500 muf

$F$ muf

F muf

$\frac{F}{E}-\frac{\text { muf }}{\text { muf }}$

$M 3500$

$12-M 3000$

F2-M 1500

FI-M 3000

M

$* F_{2} M 3000$

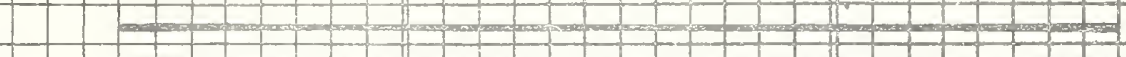

* $\mathrm{F}_{2} \mathrm{M} 4000$

$\mathrm{h}^{\prime} \mathrm{F}_{2}$

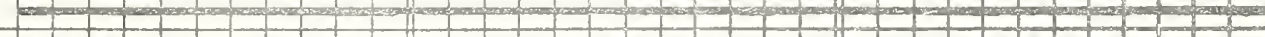

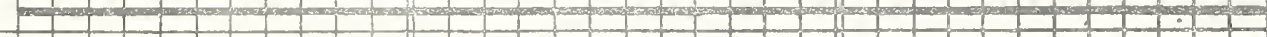

$h^{\prime} E$ -

$h^{\prime} E_{2}$

$h^{\prime} E_{S}$

$h^{\prime} E_{25}$
$h^{m} F_{2}$
$h^{m} F_{1}$
$h^{m} E$

Absorption

* Calculated by IRPL

0-1942, monthly averases.

-1943 and following, dally tabulations. 

San Juan, Puerto Rico

$\left(18.4^{\circ} \mathrm{N}, 65.1^{\circ} \mathrm{W}\right)$

IONOSPHERIC DATA RECORD

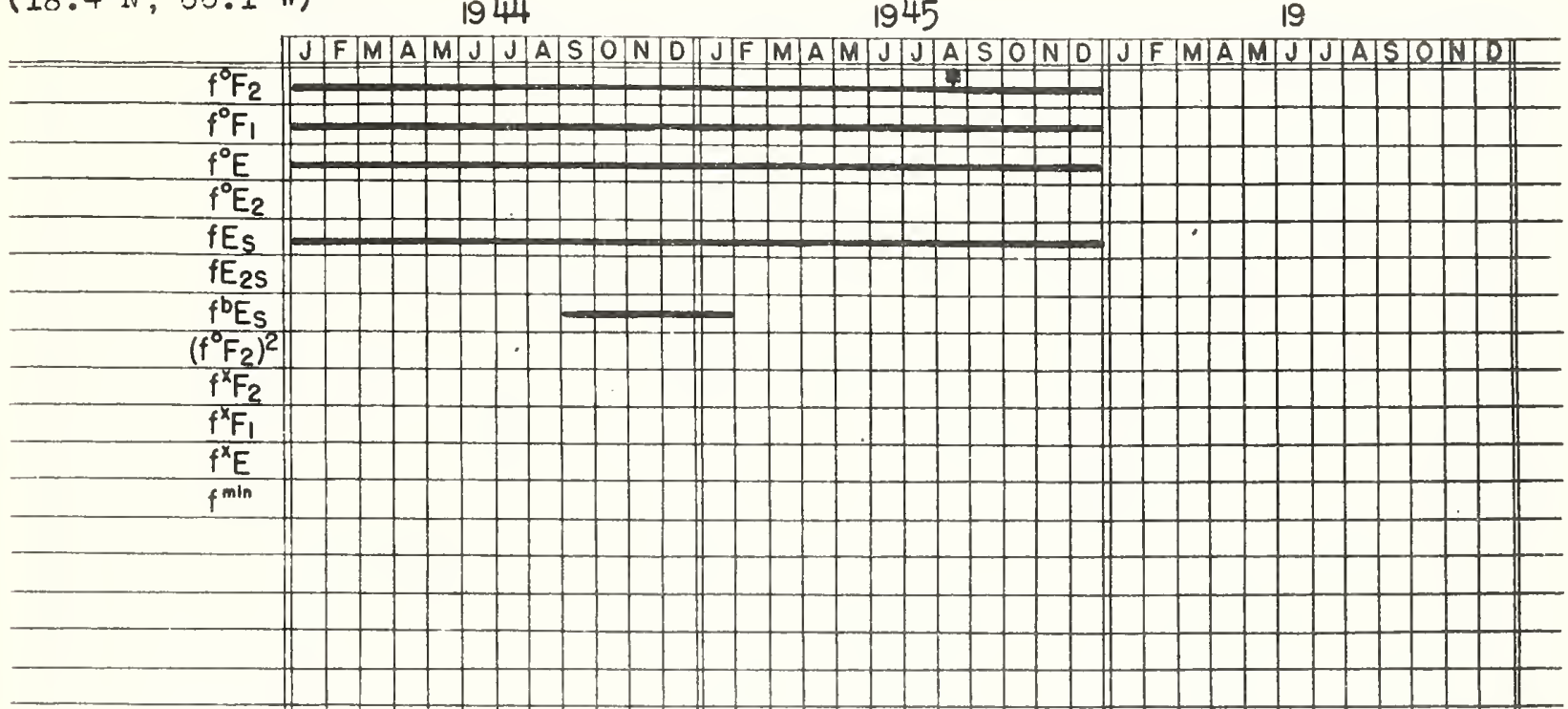

$F_{2} 3500$ muf

$F_{2} 1500$ muf

$F_{2} 3000$ muf

$F$ muf

$F_{1} 3000$ muf

$\begin{array}{ll}F & \text { muf } \\ E & \text { muf }\end{array}$

M3500

F2-M3000

$\frac{M}{M}-\frac{M}{M} 3000$

1 14000

* $\mathrm{F}_{2} \mathrm{M} 3000$

* $F_{2} M 4000$

$\mathrm{h}_{2}$

$h^{\prime} F_{1}$

h'E

$h^{\prime} E_{2}$

$h^{\prime} E_{s}$

$h^{\prime} E_{25}$

$h^{m} F_{2}$

$\frac{h^{m} F_{1}}{h^{m} E}$

1944
1945
19 

San Juan, Puerto Rico

IONOSPHERIC DATA RECORD

(18.40\%, 66.10\%)

1941

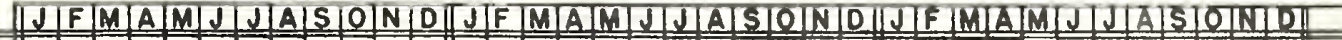

F23500muf

$\mathrm{F}_{2} 1500 \mathrm{muf}$

$F$ muf

F muf

$F$ muf

E muf

F $M 3500$

$F M 3000$

$F M 1500$

F M3000

F $M 4000$

* $F_{2} M 3000$

* $F_{2} M 4000$

$h^{\prime} \mathrm{F}_{2}$

$h^{\prime} F_{1}$

h'E

$h^{\prime} E_{2}$

$h^{\prime} E_{S}$

$h^{\prime} E_{2 s}$

$h^{m} F_{2}$

$h^{m} F_{1}$ 

Slough, Fingland

IONOSPHERIC DATA RECORD

$(51.50 \mathrm{~N}, 0.60 \mathrm{w})$

1944

1945

19

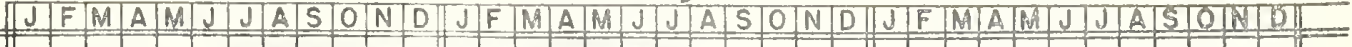

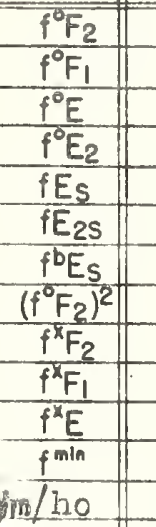

\begin{tabular}{|ll|}
\hline$F$ & muf \\
\hline$F$ & muf \\
\hline$F$ & muf \\
\hline$F$ & muf \\
\hline$F$ & muf \\
\hline$F$ & muf \\
\hline$E$ & muf \\
\hline
\end{tabular}

M3500

$+2-M 3000$

$M$

M

* $\mathrm{F}_{2} \mathrm{M} 3000$

* $\mathrm{F}_{2} \mathrm{M} 4000$

$h^{\prime} F_{2}$

$h^{\prime} F_{1}$

hE

$h^{\prime} E_{2}$

$h^{\prime} E_{S}$

$h^{\prime} E_{25}$

$\frac{h^{m} F_{2}}{h^{m} F_{1}}$

$h^{m} E$

I

Absorption

* Calculated by IRPL

Atonthly avernges only. 



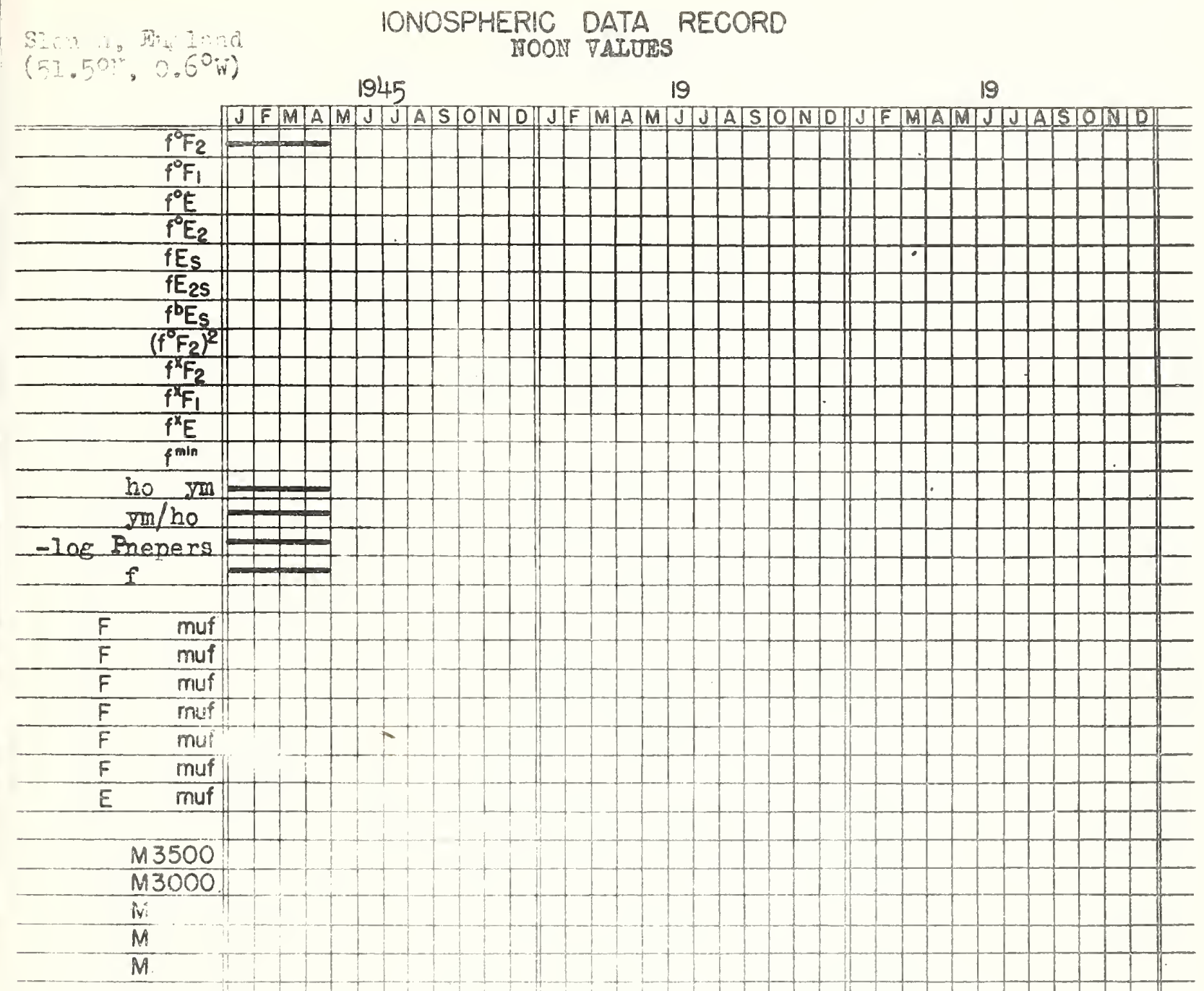

$$
\begin{array}{r}
* F_{2} M 3000 \\
* F_{2} M 4000 \\
\hline h^{\prime} F_{2} \\
\hline h^{\prime} F_{1} \\
h^{\prime} E \\
h^{\prime} E_{2} \\
h^{\prime} E_{s} \\
\hline h^{\prime} E_{2 S} \\
\hline h^{m} F_{2} \\
\hline h^{m} F_{1} \\
\hline h^{m} E
\end{array}
$$$$
\text { HOON JALURS }
$$

19 

Slough, Ingland

$\left(51.50 \mathrm{~N}, 0.5^{\circ} \mathrm{W}\right)$
IONOSPHERIC DATA RECORD HOON VALUURS

1942

1943
1944

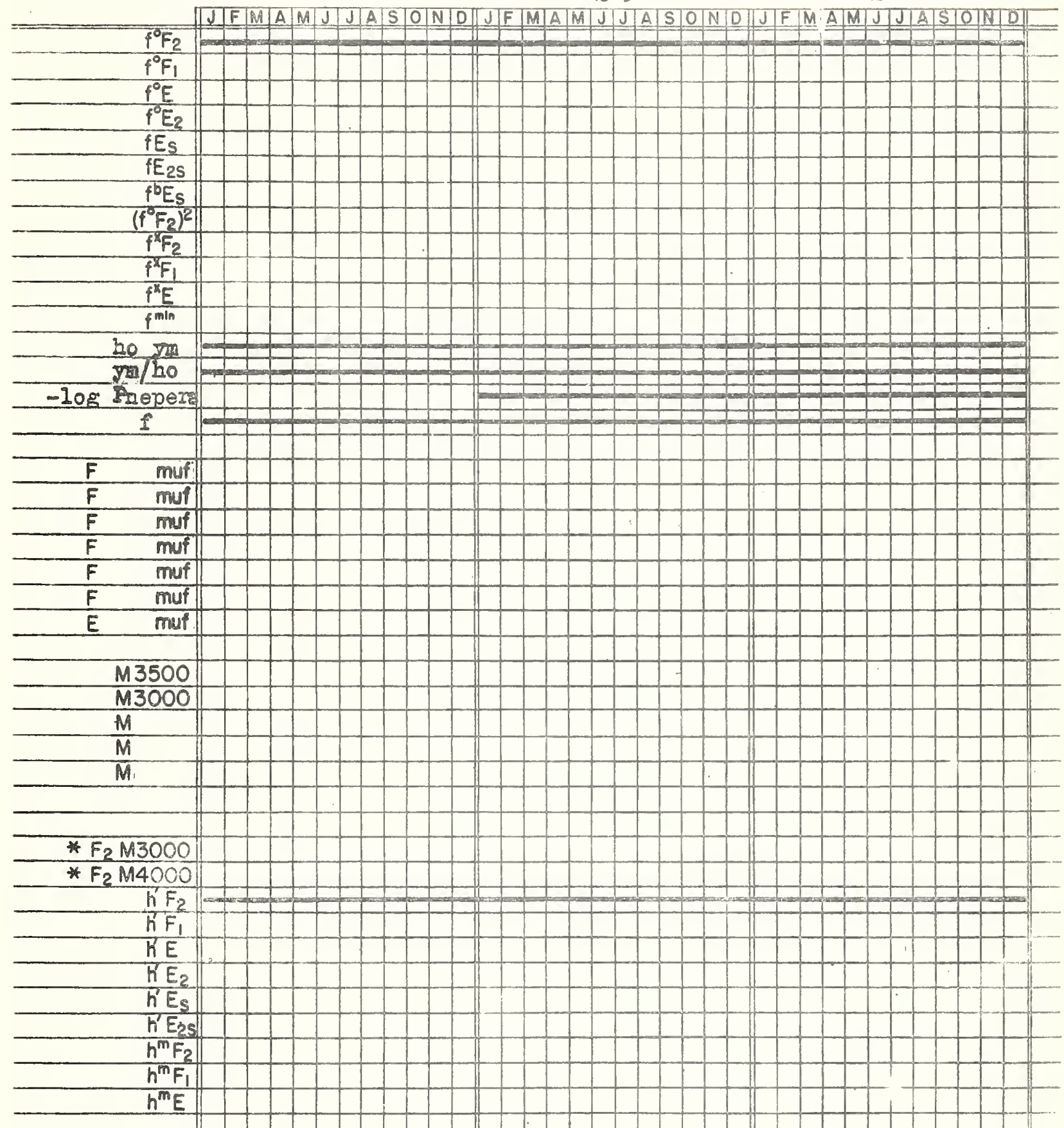
Absorpilon

* Colculated by IRPL

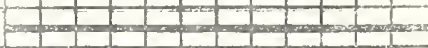





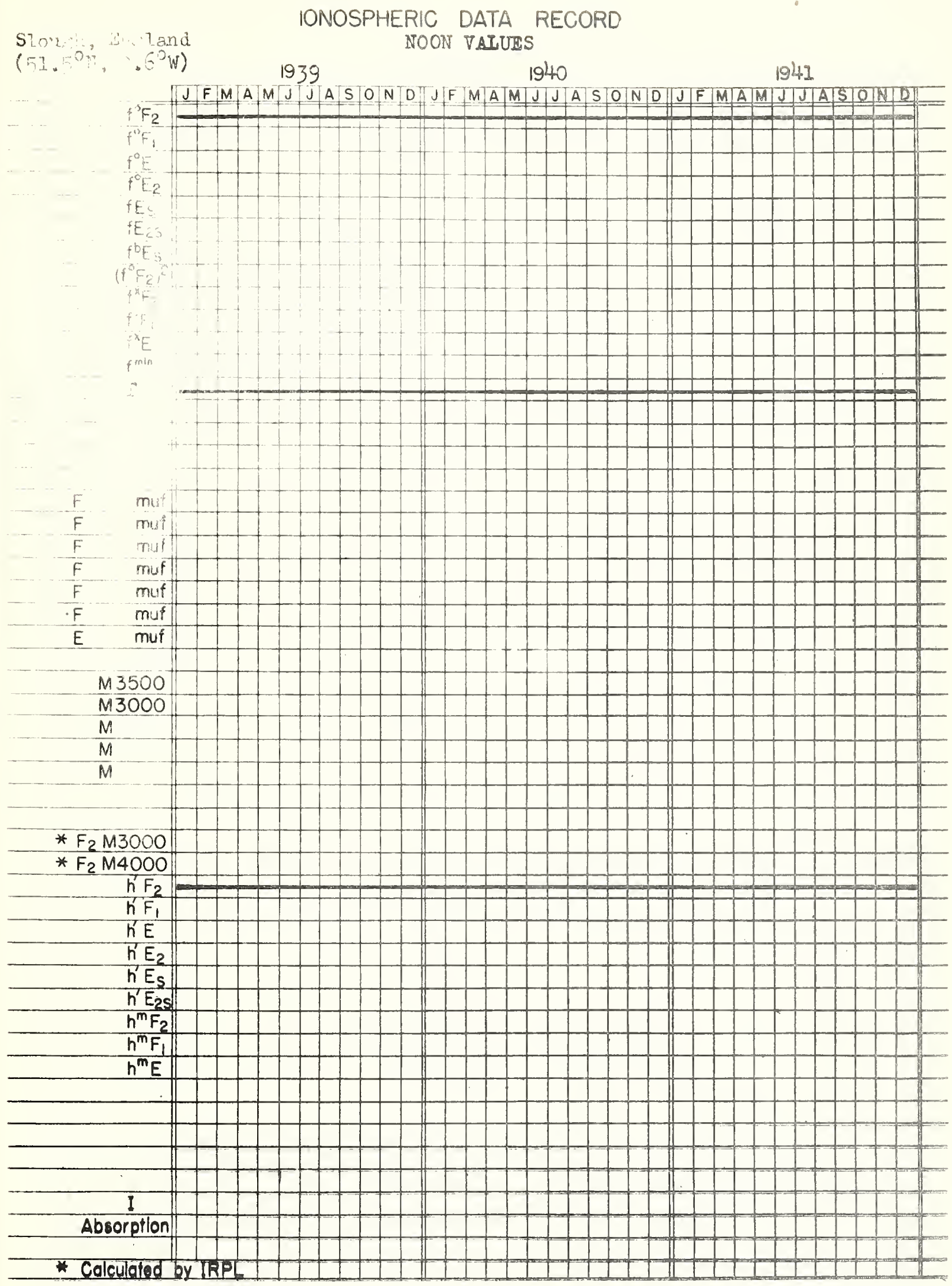



Slutsk, U.S.S.R.

$\left(59.7^{\circ} N_{3}, 30.5^{\circ}\right.$ ? $)$

1940

1941

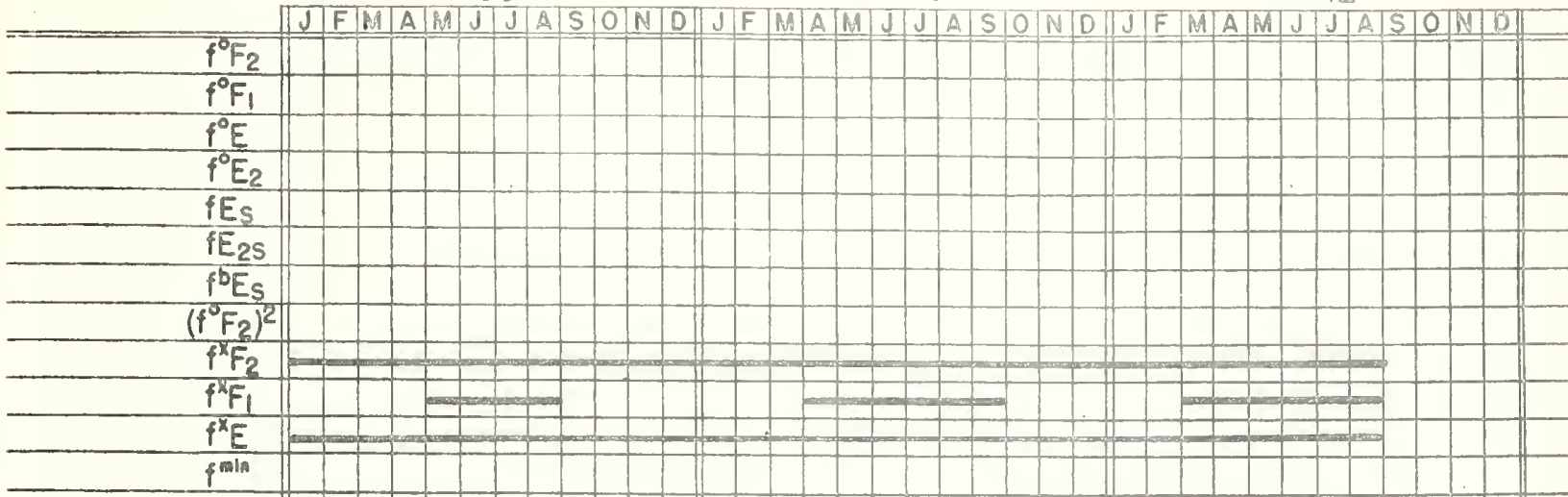

$\frac{M 3500}{M 3000} \frac{M}{M}$

* F $F_{2} M 3000$

* $\mathrm{F}_{2} \mathrm{M} 4000$

$h^{\prime} F_{2}+2$

$h^{\prime} F_{1}-\frac{1}{h}$

$h^{\prime} \mathrm{E}$

$h^{\prime} E_{2}$

$h^{\prime} E_{S}$

$h^{\prime} E_{2 s}$

$h^{m} F_{2}$

$h^{m} F_{1}$

$h^{\text {min } E}$ 

Snainton, Ingrand

IONOSPHERIC DATA RECORD

$\left(54.2^{0} \mathrm{~N}, 0.6^{\mathrm{O}} \mathrm{W}\right)$

1944

19

19

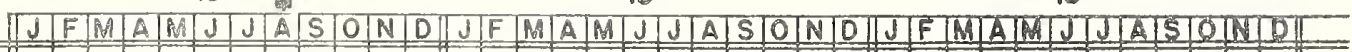

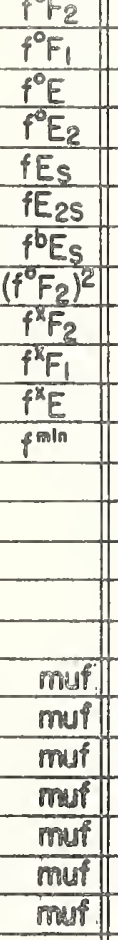

M3500

$M 3000$

M

M

M

* $F_{2} M 3000$

* F $\mathrm{F}_{2} \mathrm{M} 4000$

$\mathrm{h}_{2}$

KF

RE

HE

$h^{\prime} E_{s}$

$h^{\prime} \mathrm{Es}_{\mathrm{S}}$

$h^{\text {min }} F_{2}$

$\frac{h^{m} F_{1}}{h^{m} E}$ 1 


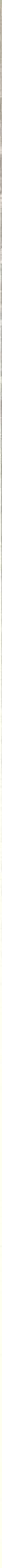




\section{IONOSPHERIC DATA RECORD}

Spitsbergen, Norway

$\left(78.2^{\circ}, 15.6^{\circ} \mathrm{m}\right)$

1942

19

19

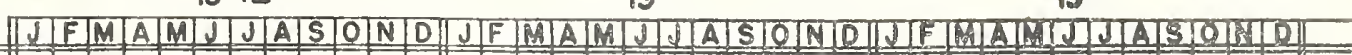

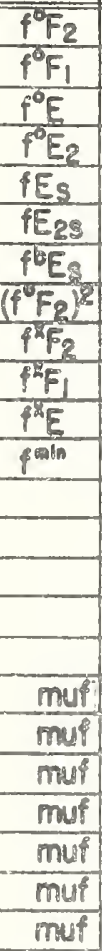

M3500

M3000

$\mathrm{M}$

M

Mi

* Fr M3000

* Fr M4000

$h F_{2}$
$h F_{1}$
$h E^{2}$
$h E_{2}$
$h^{n} E_{5}$
$h^{n} E_{3}$
$h^{n} F_{2}$
$h^{n} E_{1}$
$h^{n} E_{5}$

1212

(1) 

Sverdlovsk, U.S.S.R. IONOSPHERIC DATA RECORD

$\left(56.7^{\circ \mathrm{NP}}, 61.1^{\circ} \mathrm{E}\right)$ 41944

1945

19

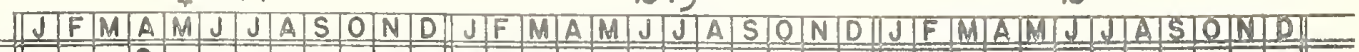

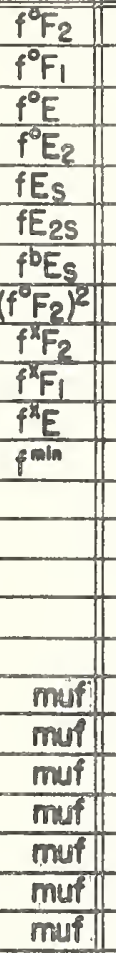

$\frac{M 3500}{M 3000} \frac{M}{M}$

* F $M 3000$

* $F_{2} M 4000$

$h^{\prime} F_{2}$

$h^{\prime} F_{1}$

hE

KE

$h^{\prime} E_{s}$

$h^{\prime} E_{25}$

$h^{m} F_{2}$

$h^{\text {min }} F_{1}$

$h^{m} E$

Absorption

* Calculated by IRPL

- For all months POPR dailyohourly tabulations. Others monthly average日.

Olpr11 1944, noon for2 and rain fort2 for 20 dags only. 
Syrachy, 51c11\%

IONOSPHERIG DATA RECORD

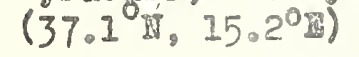

1942

1943

19

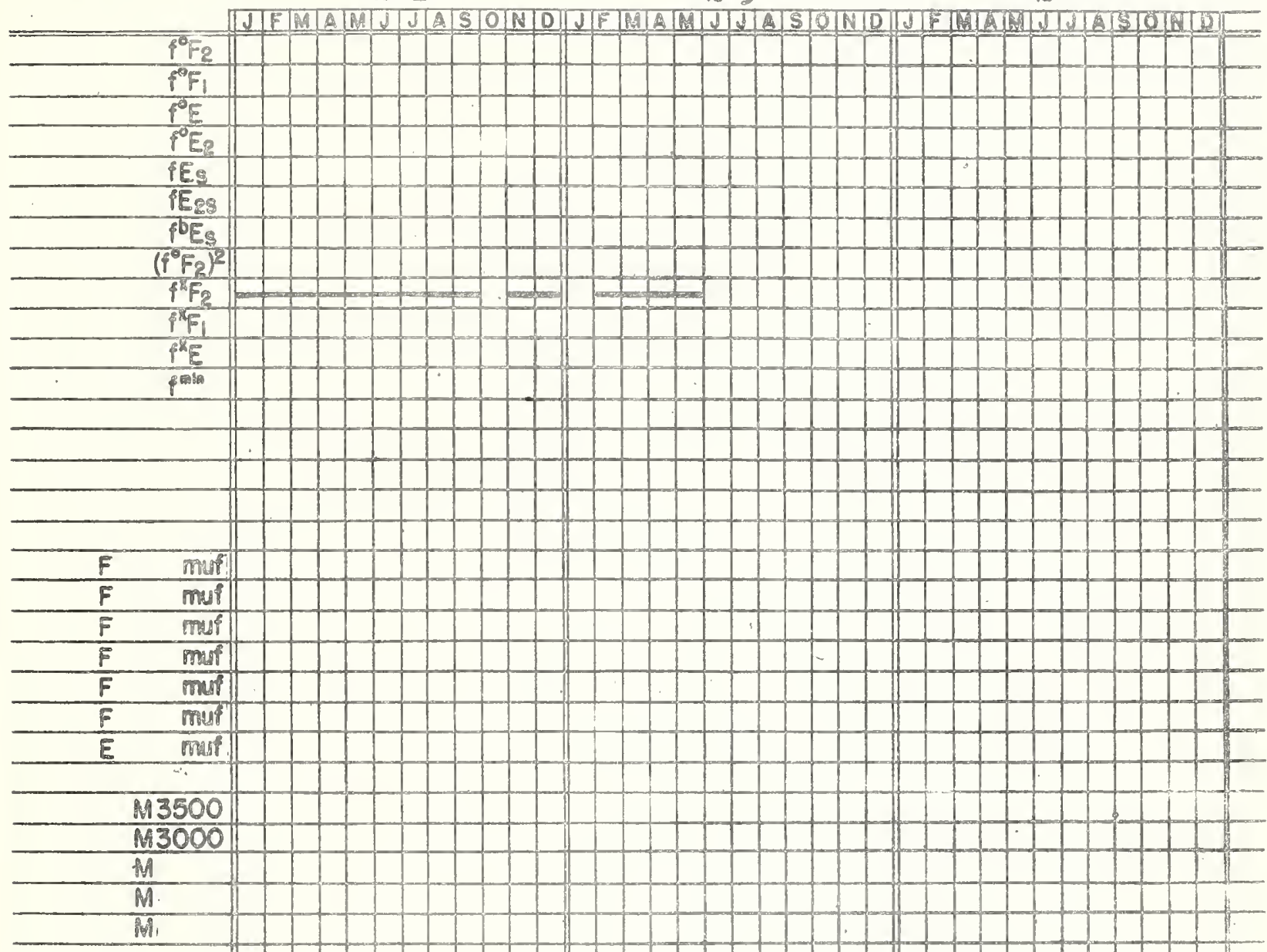

* F $\mathrm{F}_{2} 3000$

* F $F_{2} M 4000$

$h^{\prime} F_{2}$

hFi

RE

HE.

$h^{\prime} E_{5}$

$h^{\top} \mathrm{E}_{2}$

$h^{m} F_{2}$

$h^{m} F_{1}$

$h^{m}$ E

$\frac{1}{\text { Absorstion }}$

* Colculoted by Mist

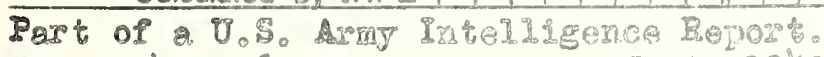

Jan. 1942, $16 \mathrm{mog} 100 \mathrm{y}$.

JuIy 1942, 1w11 only.

gapto 1942: $9005 \%$ \%

跑y $2943.26-32$ only. 



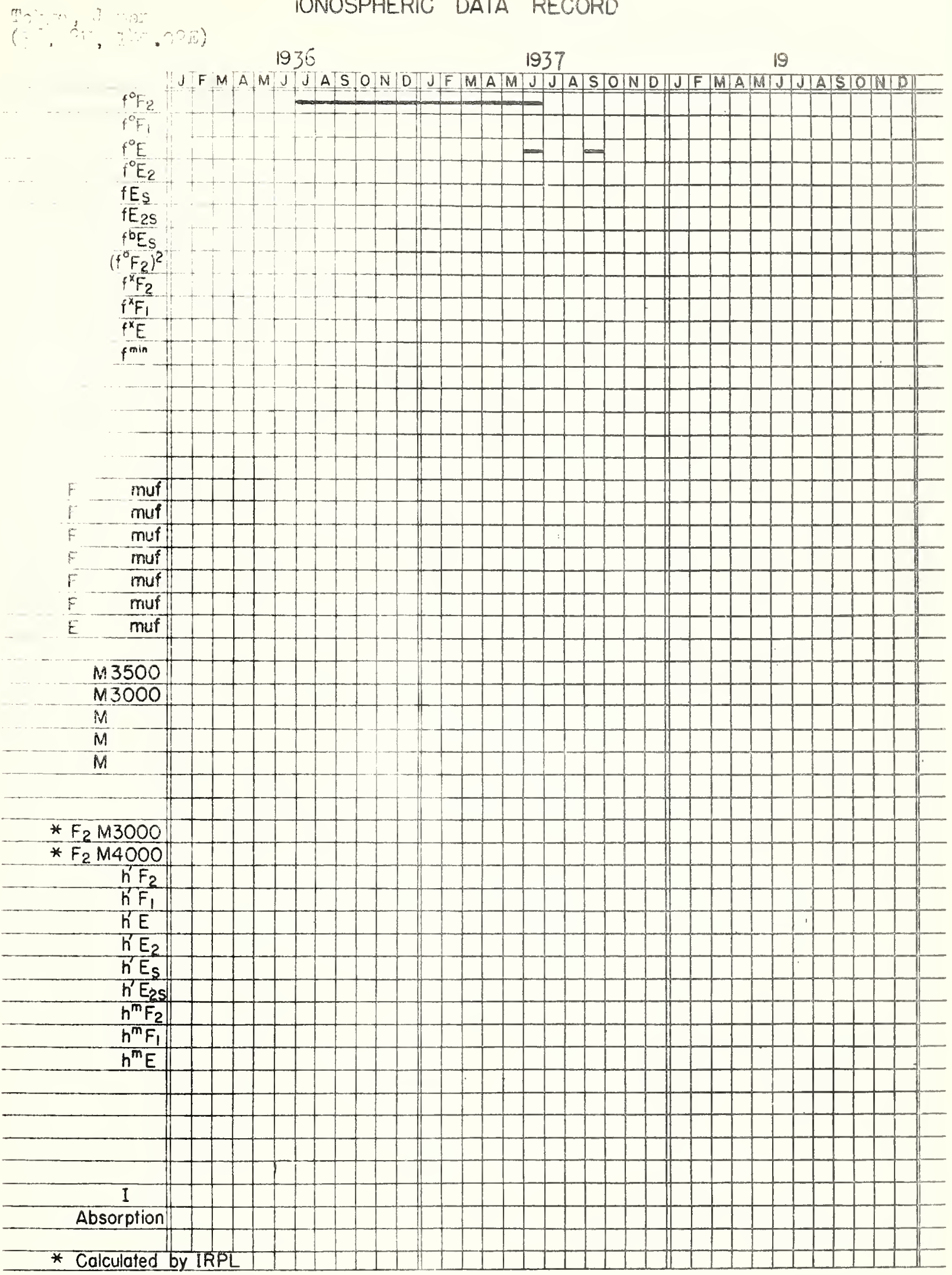

Monthly everares on?y. 

Tomsk, U.S.S.R.

$\left(56.4^{\circ} \mathrm{N}, 85.0^{\circ} \mathrm{T}\right)$

\section{IONOSPHERIC DATA RECORD}

1944

1945

1943

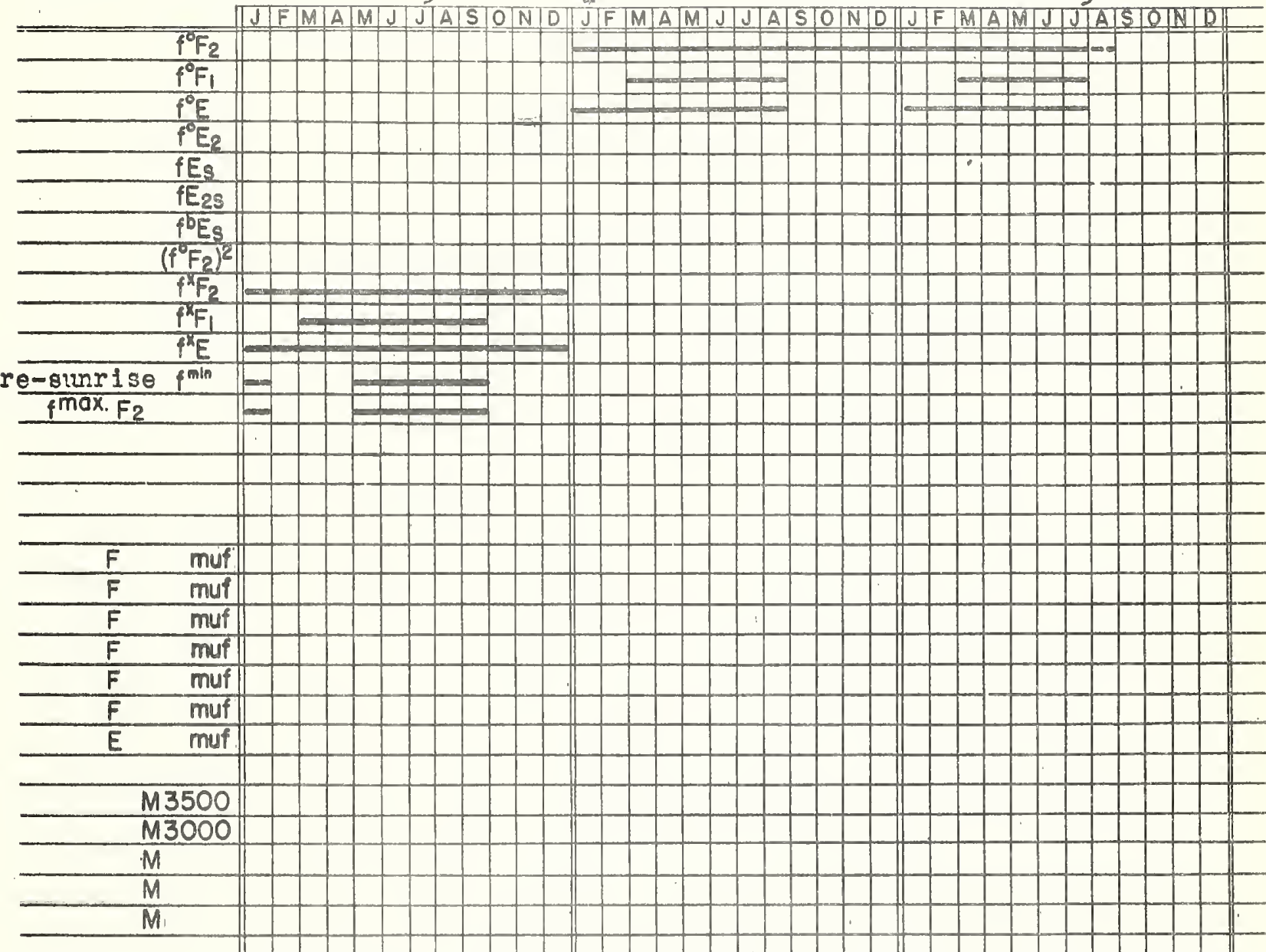

* $F_{2} M 3000$

* F $\mathrm{F}_{2} \mathrm{M} 4000$

$h^{\prime} \mathrm{F}_{2}$

$h F_{1}$

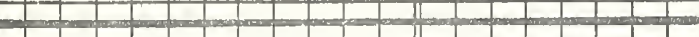

-

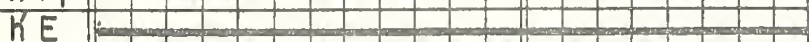

$-1-1-1$

h'

$h^{\prime} E_{s}$

$h^{\prime} E_{2 s}$

$h^{m} F_{2}$

$h^{m} F_{1}$

$h^{m} E$

I

Absorption

* Calculated by IRPL

Han. through June 1944, daily-hourly tabulations fop.

other values, monthly arerages. 

Tomek, 0.5 .5 .8$.

$\left(56.4^{\circ}, 85.0^{\circ}\right.$ 窟)

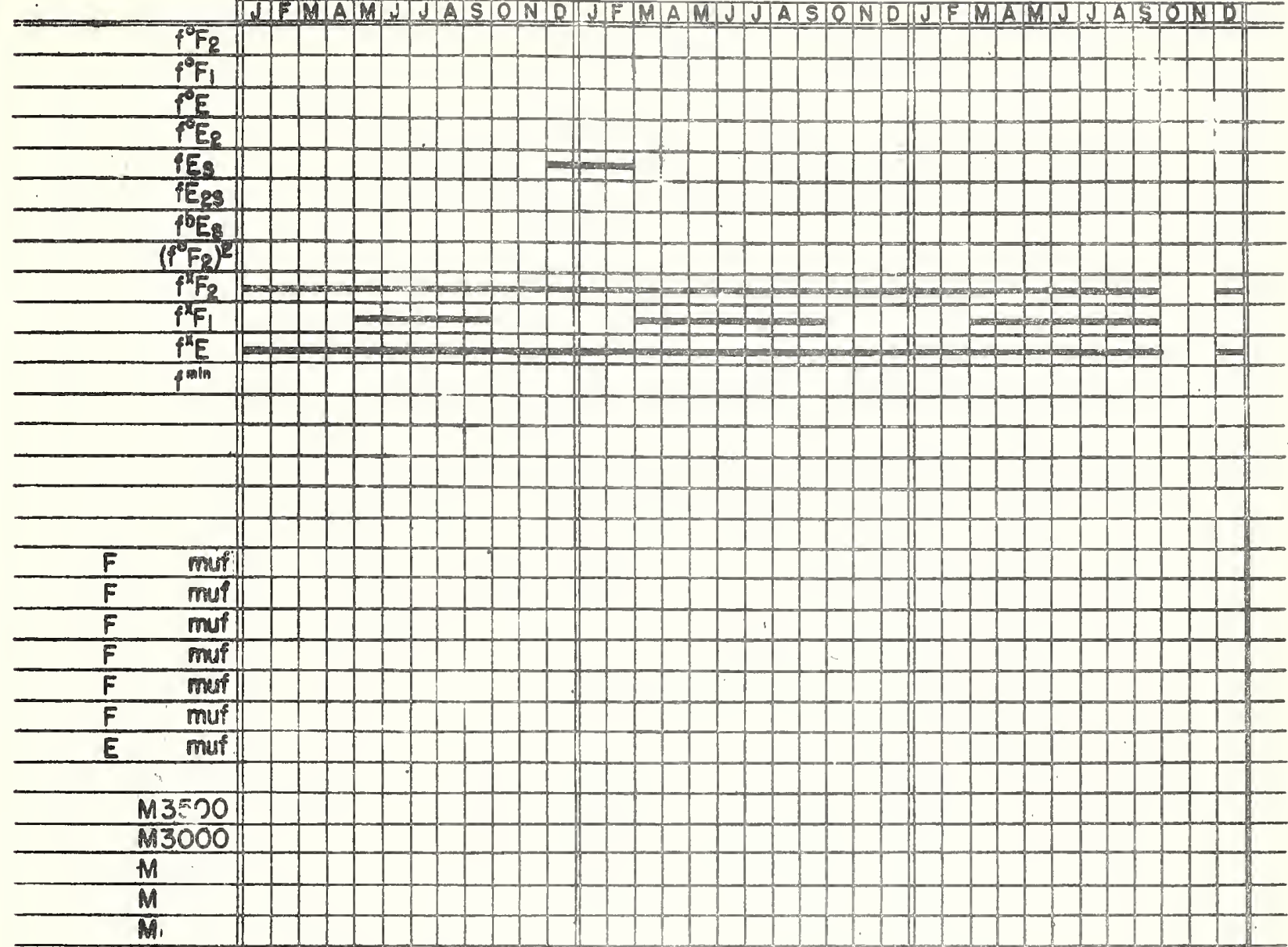

* F $F_{2} M 000$

* Fr M4000

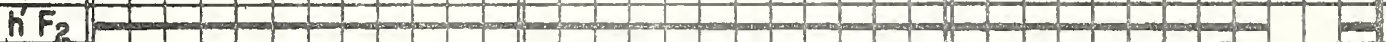

$K F_{1}$

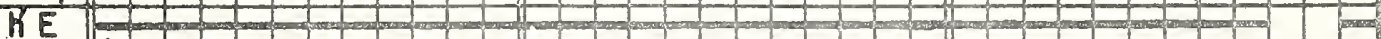

RE.

$h^{\prime} E_{s}$

$h^{\prime} E_{s s}$

$h^{m} F_{2}$

$\frac{h^{m} F_{1}}{h^{m} E}$

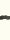

I

Absorption

* Calculatad by IRPL

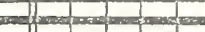

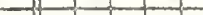



Tomsk, U.S.S.R.
$\left(56.4^{6} \mathrm{NT}, 85.0^{\circ} \mathrm{E}\right)$
1937
1938
1939

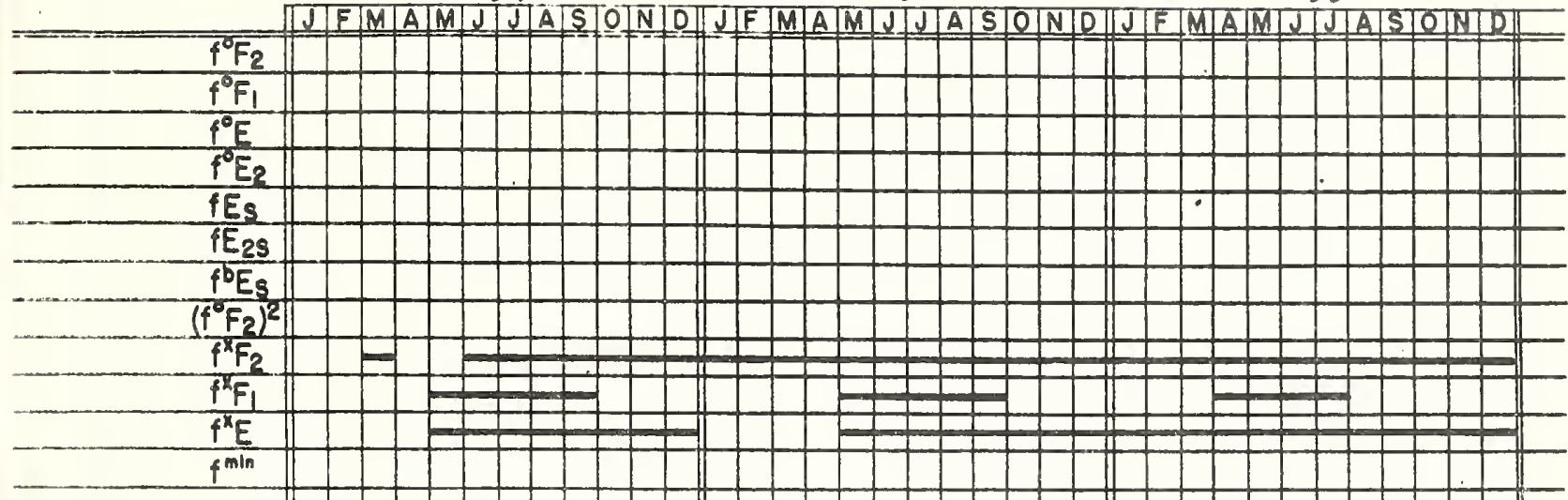

\begin{tabular}{cc|}
\hline$F$ & muf \\
\hline$F$ & muf \\
\hline$F$ & muf \\
\hline$F$ & muf \\
\hline$F$ & muf \\
\hline$F$ & muf \\
\hline$E$ & muf
\end{tabular}

$\frac{M 3500}{M 3000} \frac{M}{M}$

* Fo $M 3000$

* F $2 M 4000$

\begin{tabular}{l|}
$h^{\prime} F_{2}$ \\
$h^{\prime} F_{1}$ \\
$h^{\prime} E$ \\
$h^{\prime} E_{2}$ \\
$h^{\prime} E_{s}$ \\
$h^{\prime} E_{2 s}$ \\
$h^{m} F_{2}$ \\
$h^{m} F_{1}$ \\
$h^{m} E^{m}$
\end{tabular}
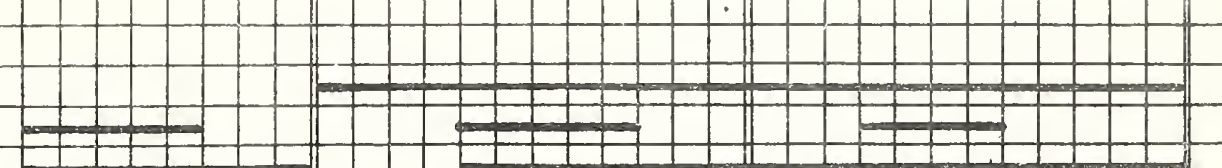

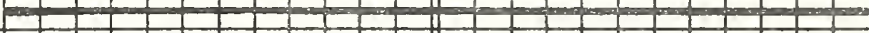
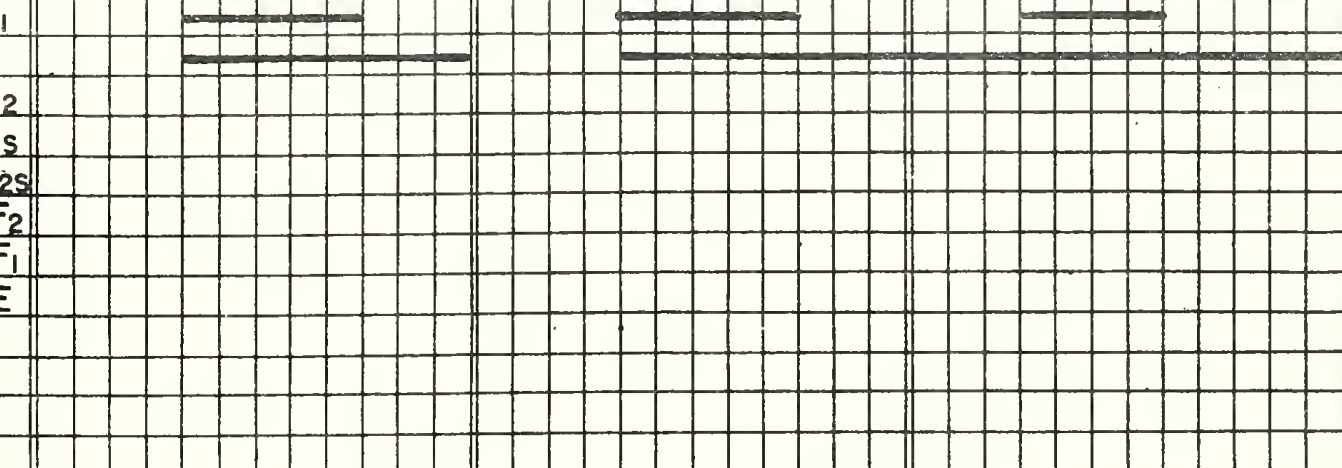

IONOSFIERIC IATA RECORE

Trinicad, Brit, Wost Indies

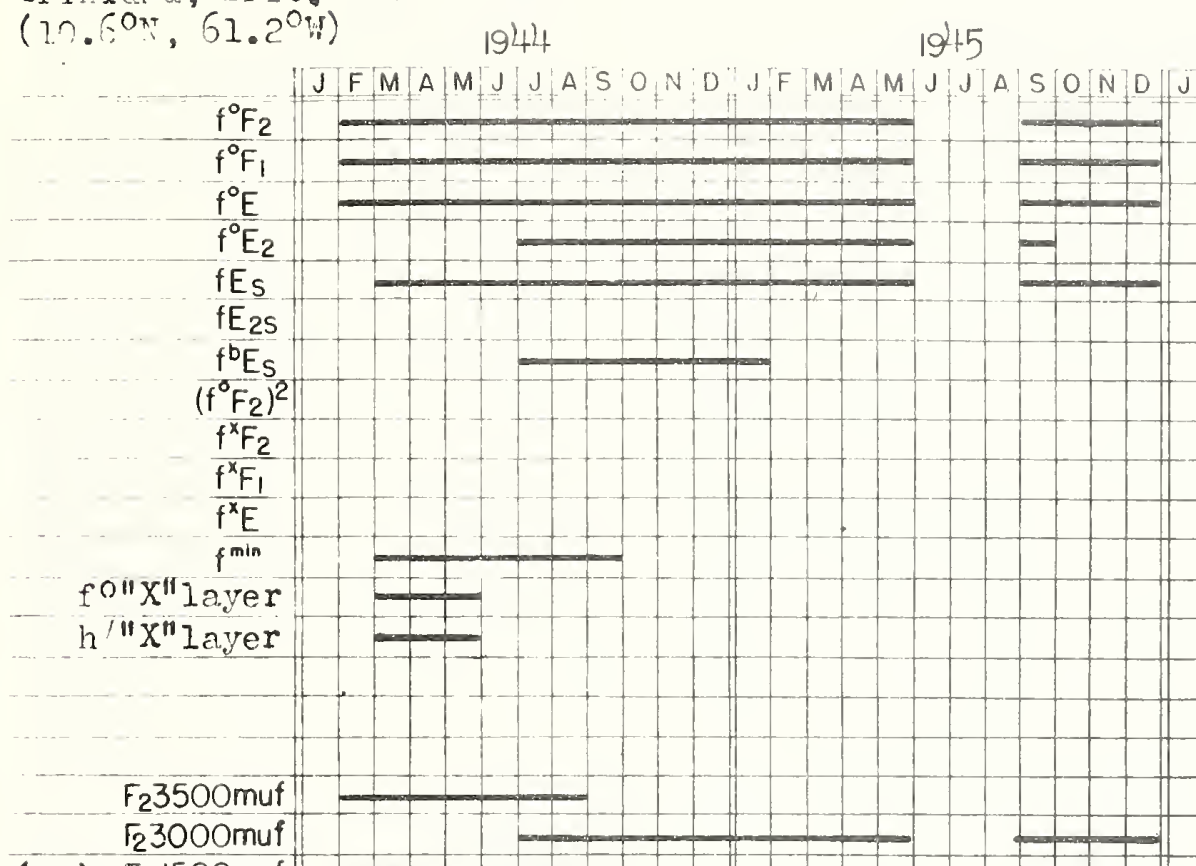

(ni) F21500muf

(mi) $\mathrm{F}_{2}$ lOOOMuf

(ni) $\mathrm{F}_{2} 500 \mathrm{muf}$

(mi) Eslooomuf

Fir -1.3500

F2 $-M 3000$

$M$

(mi) $\frac{F 2-M}{M} 1000$

* $\mathrm{F}_{2} \mathrm{M} 3000$

* $\mathrm{F}_{2} \mathrm{M} 4000$

$h^{\prime} F_{2}$

$h^{\prime} F_{1}$

h'E

$h^{\prime} E_{2}$

$h^{\prime} E_{S}$

$h^{\prime} E_{2 s}$

$h^{m} F_{2}$
$h^{m} F_{1}$
$h^{m} E$

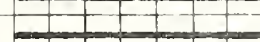

\begin{tabular}{l|l|l|}
1 \\
-1
\end{tabular}

$+2+2$

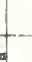

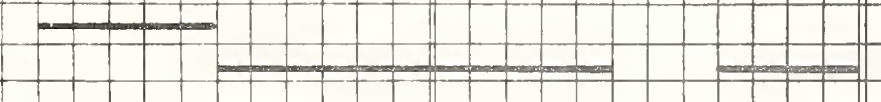

$\frac{1}{1+1+1}$
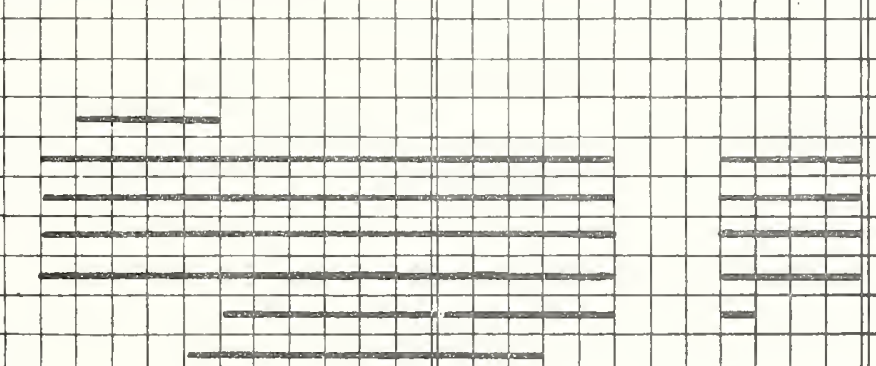

Tromso, Norway.
$\left(69.6^{\circ} \mathrm{N}, 19.0^{\circ} \mathrm{E}\right)$

IONOSPHERIC DATA RECORO

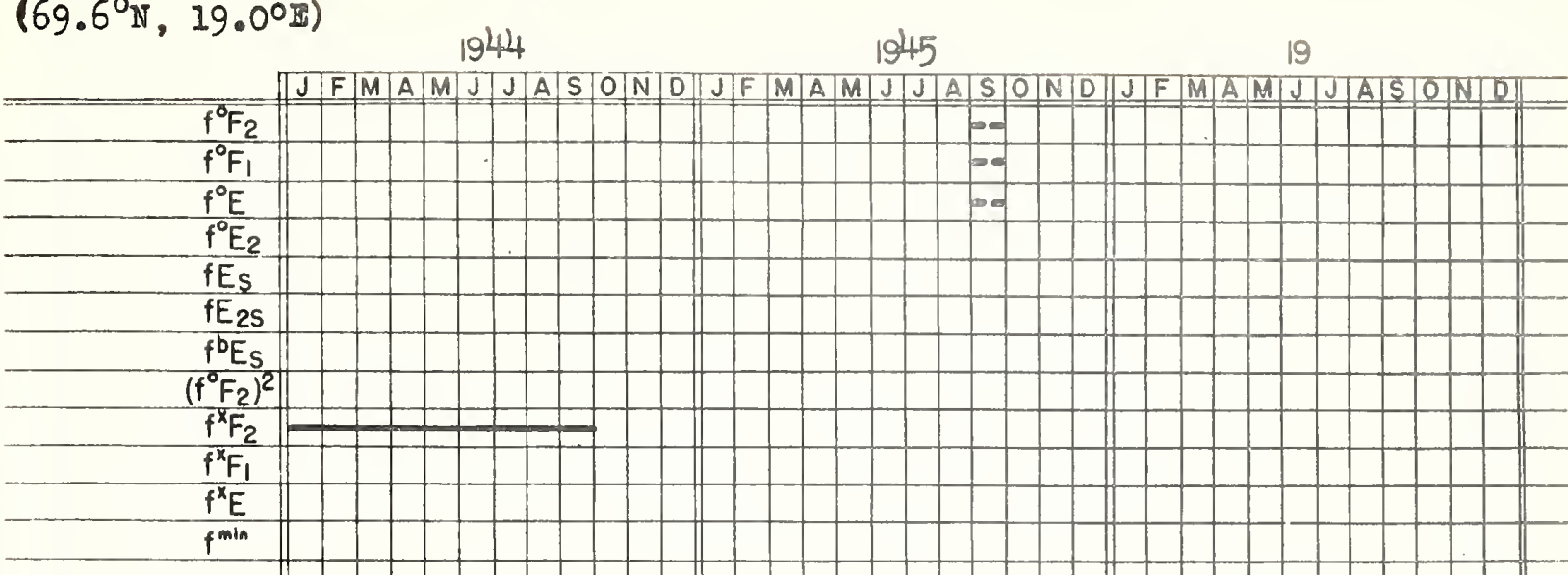

\begin{tabular}{|ll|}
\hline$F$ & muf \\
\hline$F$ & muf \\
\hline$F$ & muf \\
\hline$F$ & muf \\
\hline$F$ & muf \\
\hline$F$ & muf \\
\hline$E$ & muf \\
\hline
\end{tabular}

M3500

M3000

$M$

M

M

* $\mathrm{F}_{2} \mathrm{M} 3000$

* $\mathrm{F}_{2} \mathrm{M} 4000$

$\mathrm{h}^{\prime} \mathrm{F}_{2}$

$h^{\prime} F_{1}$

hE

$h^{\prime} E_{2}$

$h^{\prime} E_{S}$

$h^{\prime} E_{2 s}$

$h^{m} F_{2}$

$h^{m} F_{1}$

$h^{m} E$ 
Tromso, Norway,

IONOSPHERIC DATA RECORD

$\left(69.6^{\circ} \mathrm{N}, 19.0^{\circ} \mathrm{R}\right)$

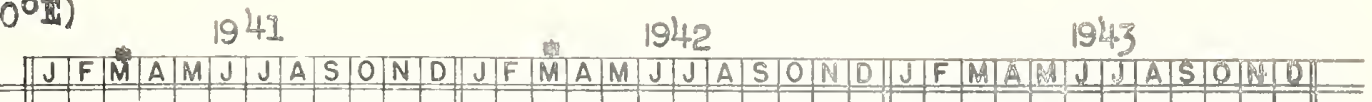

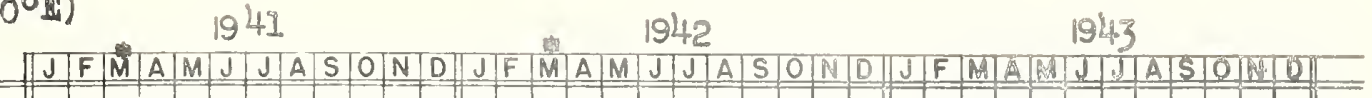

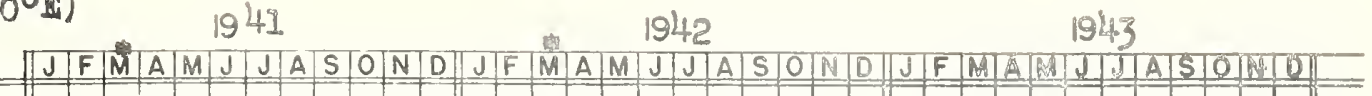

f $f$ $f^{\circ} F_{1}$

$f^{\circ} E$

fEs

$\mathrm{fE}_{2 S}$

$f^{b} E_{S}$

$f^{\mathrm{X}} \mathrm{F}_{2}$

$f^{x} F_{1}$

$f^{x} F_{E}$

$=$

f

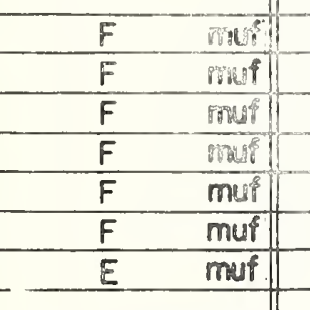

$M 3500$
$M 3000$
$M$
$M$

* $\mathrm{F}_{2} \mathrm{M} 3000$

* $\mathrm{F}_{2} \mathrm{M} 4000$

$h^{\prime} F_{2}$

$h F_{1}$

h'E

$h^{\prime} E_{2}$

$h^{\prime} E_{S}$

$h^{\prime} E_{2 s}$

$h^{6} F_{2}$

$h^{m} F_{1}$

$h^{m} E$
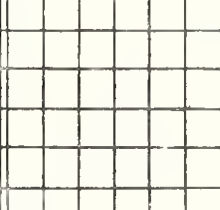

Victoria Beech, Canada IONOSPHERIC DATA RECORD

$\left(50.8^{\circ} \mathrm{N}, 96.5^{\circ} \mathrm{W}\right)$

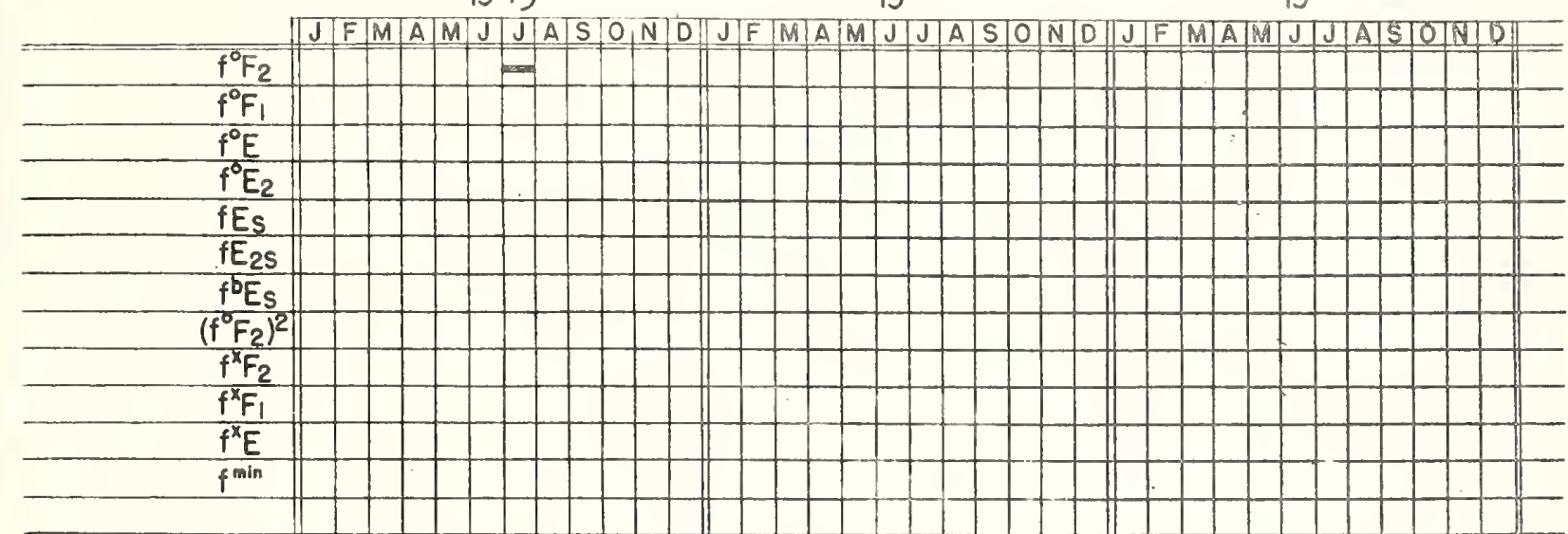

\section{M3500 \\ M3000 \\ $M$ \\ M \\ M.}

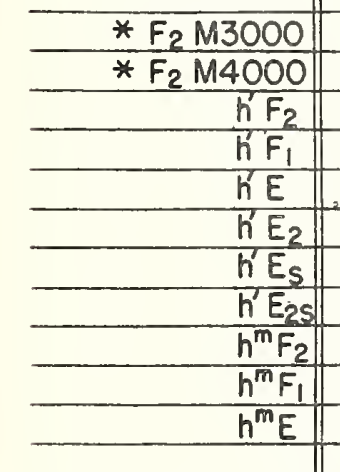


Washington, D. C.

IONOSPHERIC DATA RECORD

$\left(39.0^{\circ} \mathrm{N}, 77.5^{\circ} \mathrm{W}\right)$

1945

19

19

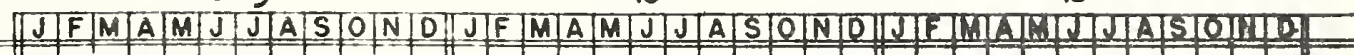

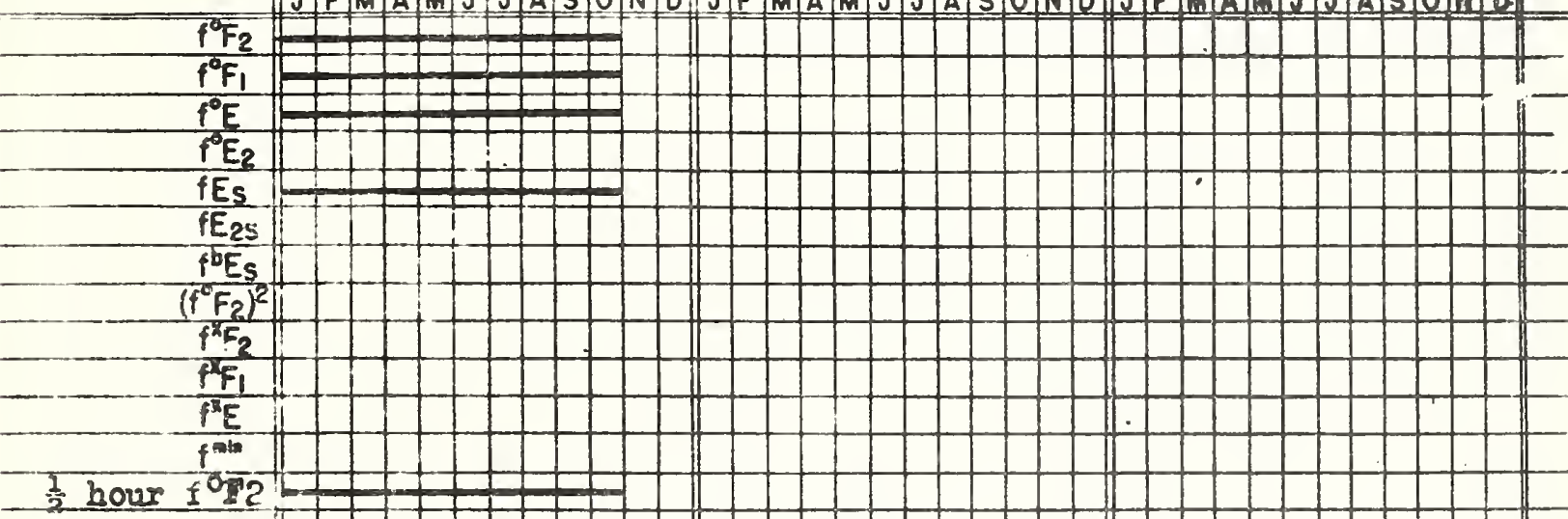

\begin{tabular}{ll}
$F$ & muf \\
$F$ & mut \\
$F$ & muf \\
$F$ & muf \\
$F$ & muf \\
$F$ & muf \\
$E$ & muf \\
\hline
\end{tabular}

T2 $2+4300$

P2 19000

TaM 1500

1. 13000

M 1500

$\begin{array}{r}* \text { * } M 3000 \\ * F_{2} M 4000 \\ \hline h^{\prime} F_{2} \\ h^{\prime} F_{1} \\ \hline h^{\prime} E \\ h^{\prime} E_{2} \\ h^{\prime} E_{s} \\ h^{\prime} E_{2 s} \\ \hline h^{m} F_{2} \\ \hline h^{m} F_{1} \\ \hline h^{m} E\end{array}$

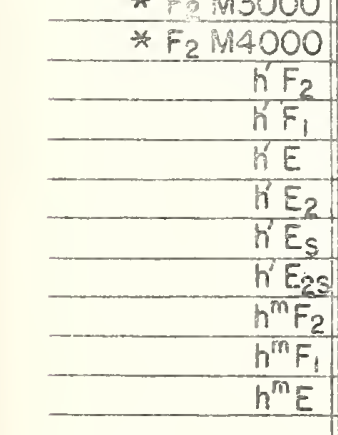

-12 -

$-1+1+1$

It 1

$+t+1$

I 7 - 

Washington, D. C.

IONOSPHERIC DATA RECORD

$\left(39.0^{\circ} \mathrm{N}, 77.5^{\circ} \mathrm{W}\right)$

1942

1943

1944

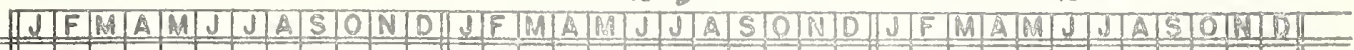

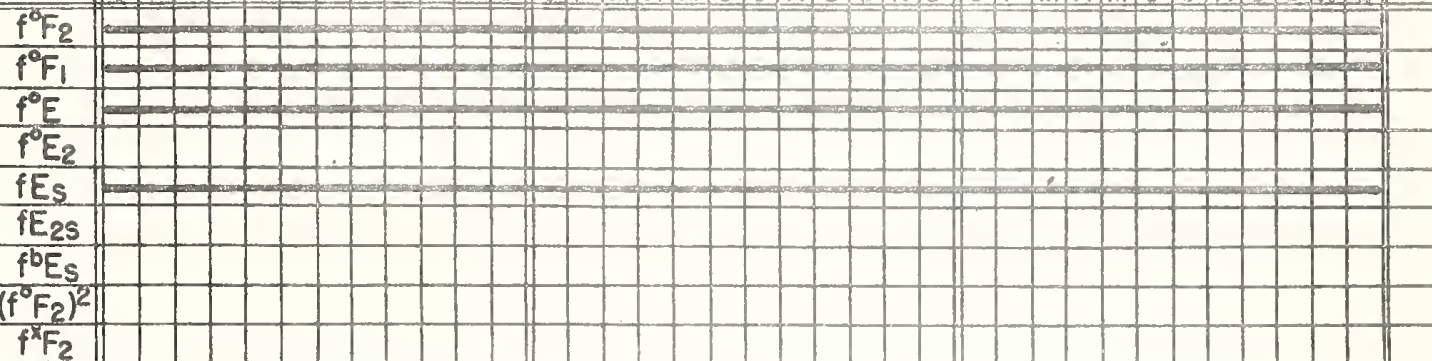

$\frac{1}{2}$ hour forte

$f^{x} F_{i}$

$f^{\mathrm{P} E}$

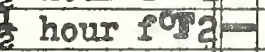

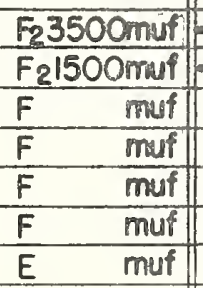

T2 $\$ 3500$

$12-13000$

$\$ 2+11500$

FI M 3000

F1 M 1500

II $M 1500$

* $F_{2} M 3000$

* $F_{2} M 4000$

$h^{\prime} \mathrm{F}_{2}$

$\vec{n}_{1} \bar{F}_{1}$

h'E

$h^{\prime} E_{2}$

$h^{\prime} E_{S}$

$h^{\prime} E_{2}$

$h^{m} F_{2}$

$h^{m} F_{1}$

$h^{m} E$

hin

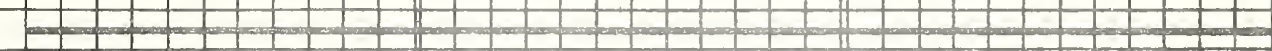

(1)

I

Absorption

* Calculated by IRPL 

Washingtoh, D. C.
$\left(39.0^{\circ} \mathrm{N}, 77.5^{\circ} \mathrm{W}\right)$
1939
1940
1941

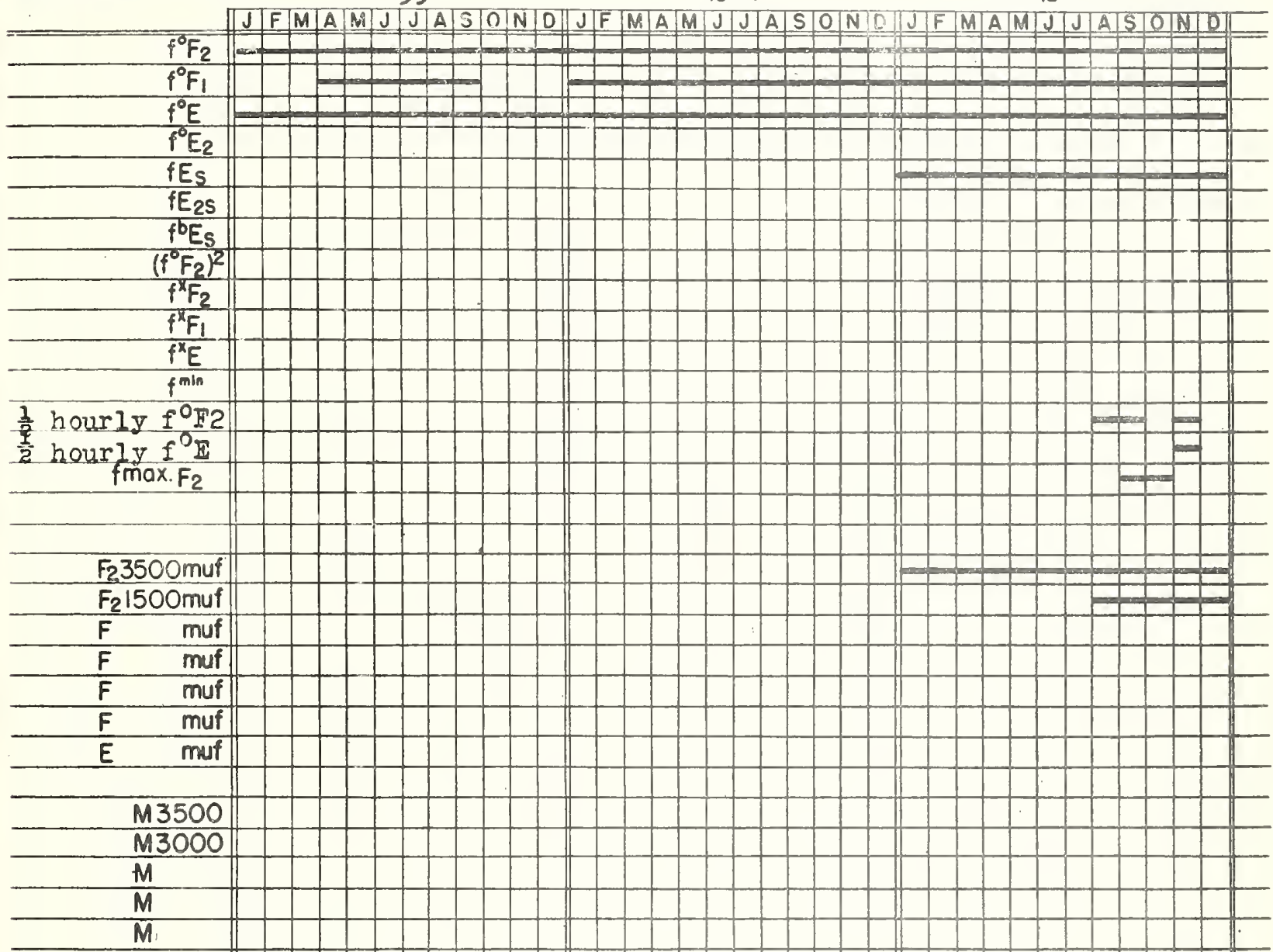

* $\mathrm{F}_{2} \mathrm{M} 3000$

* $\mathrm{F}_{2} \mathrm{M} 4000$

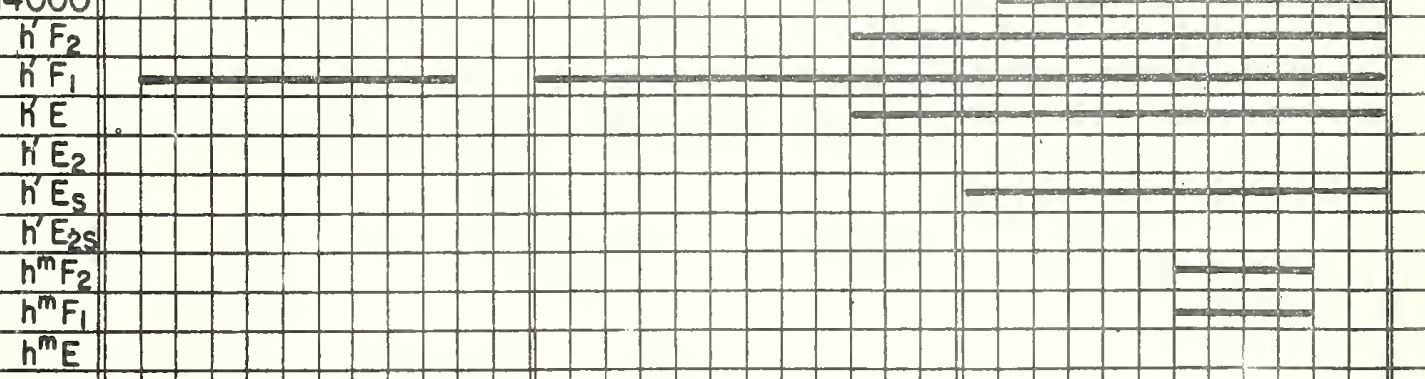

I

Absorption

* Colculated by IRPL 


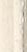


Washington, D.C. IONOSPHERIC DATA RECORD

$\left(39.00 \mathrm{~N}, 77.5^{\circ} \mathrm{W}\right)$

1937

1938

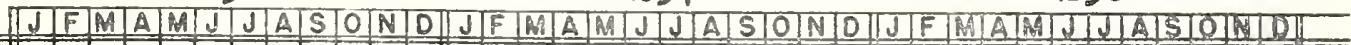

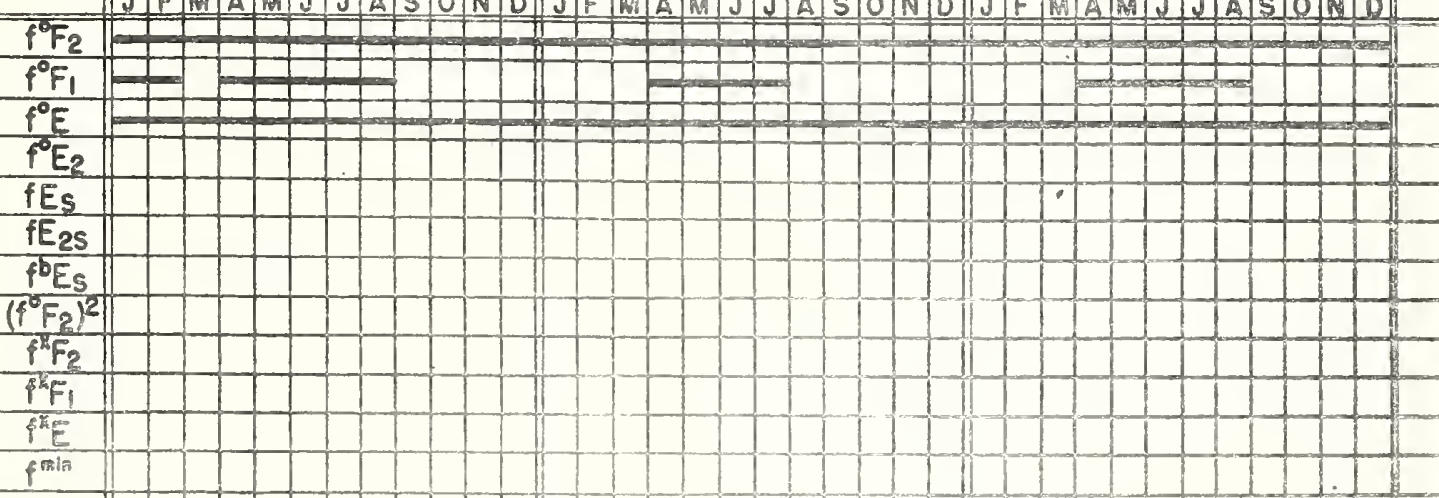

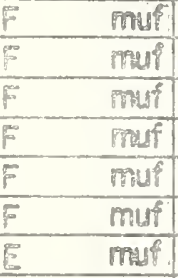

M3500

M3000

$M$

$M$

$\overline{\mathrm{N}}$

* F $F_{2} \mathrm{M} 3000$

* Fr $\mathrm{M} 4000$

$h^{\prime} F_{2}$

$h^{\prime} F_{1}$

KE

$h E_{2}$

$h^{\prime} E_{s}$

$h^{\prime} E_{2 s}$

$h^{m} F_{2}$

$h^{m} F_{1}$

$h^{m} E$

$-$

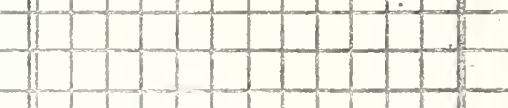

.

(

I

Absorption

* Calculated by Itit 

Washington, D. C. (39.00N, 77.50\%)
IONOSPHERIC DATA RECORD

1933

1934

1935

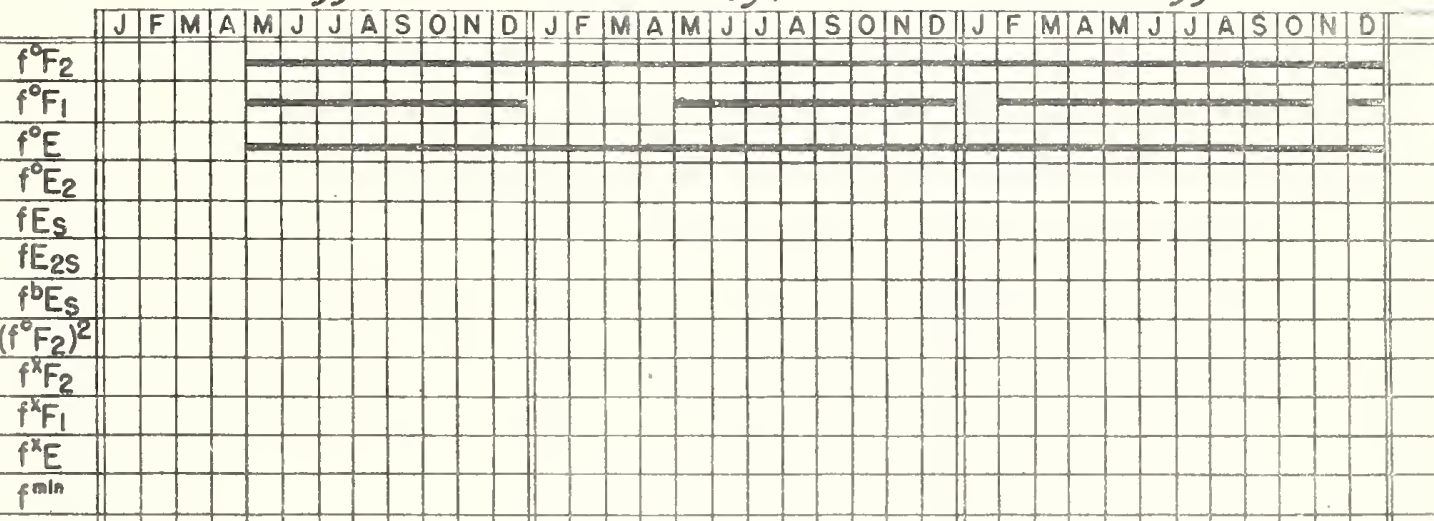

\begin{tabular}{|cr|}
\hline$F$ & muf \\
\hline$F$ & muf \\
\hline$F$ & muf \\
\hline F & muf \\
\hline$F$ & muf \\
\hline$F$ & muf \\
\hline E & muf \\
\hline
\end{tabular}

$M 3500$
$M 3000$
$M$
$M$

* $\mathrm{F}_{2} \mathrm{M} 3000$

* $\mathrm{F}_{2} \mathrm{M} 4000$

$h^{\prime} F_{2}$

h $F_{1}$

$K E_{2}$

$h^{\prime} E_{S}$

$h^{\prime} E_{25}$

$h^{m} F_{2}$

$h^{m} F_{1}$

$h^{m} E$

intion

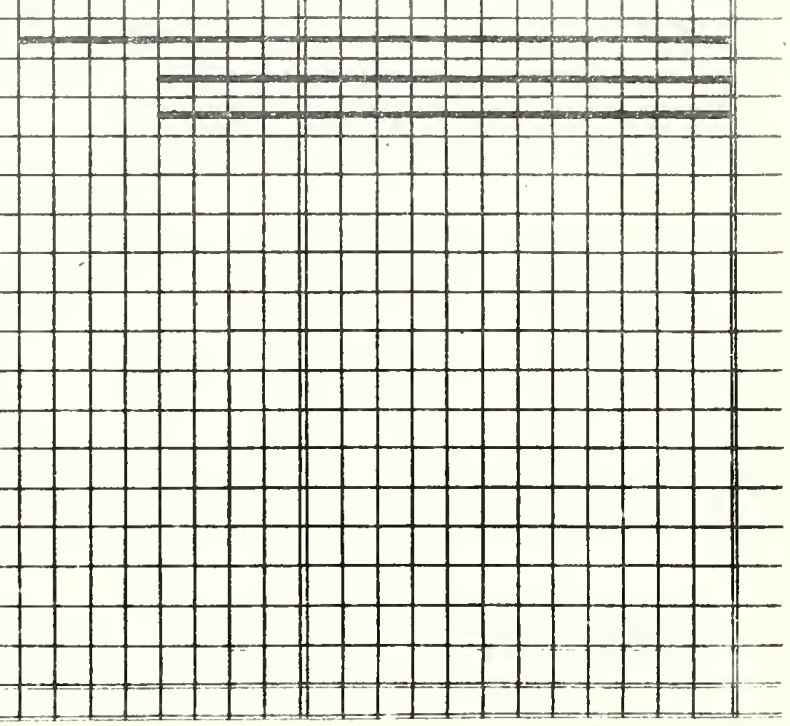





\section{Tathoroo, IONOSPHERIC DATA RECORC}

(Lat. $30.3^{\circ} \mathrm{S}, 115.9^{\circ} \mathrm{E}$ ) 1944

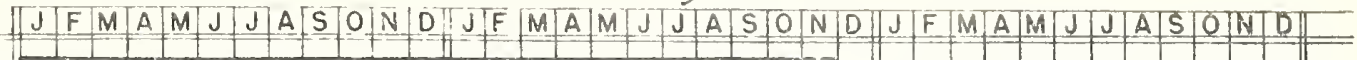

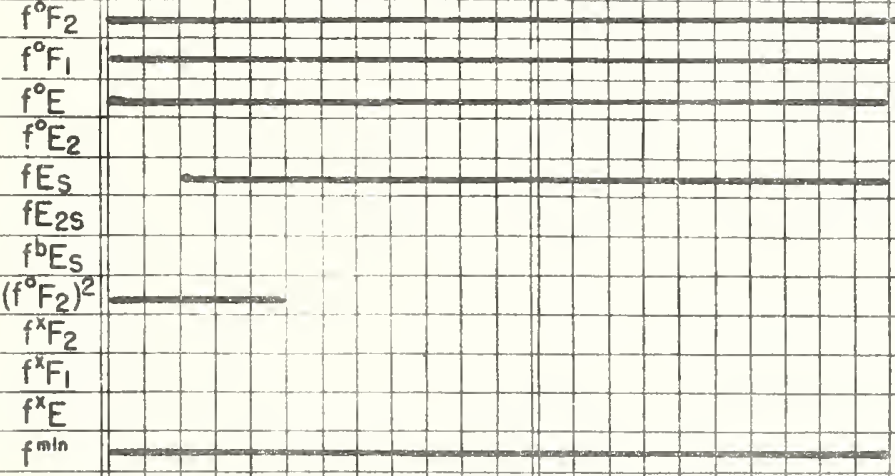

$F_{2} 3500$ muf

$\mathrm{F}_{2} 3000$ muf

$\mathrm{F}$ muf

$\mathrm{F} \quad \mathrm{mut}^{\mathrm{f}}$

F muf

F muf

E muf

$M 3500$

F2- $M 3000$

$M$

$M$

M

* $\mathrm{F}_{2} \mathrm{M} 3000$

* $F_{2} M 4000$

$h^{\prime} F_{2}$

$h^{\prime} F_{1}$

$h^{\prime} \mathrm{E}$

$h^{\prime} E_{2}$

$h^{\prime} E_{S}$

$h^{\prime} E_{2 S}$

$h^{m} F_{2}$

$h^{m} F_{1}$

his (upper)

I

Absorption

* Calculated by IRPL 
Natheroo, W. Australla

IONOSPHERIC DATA RECORD

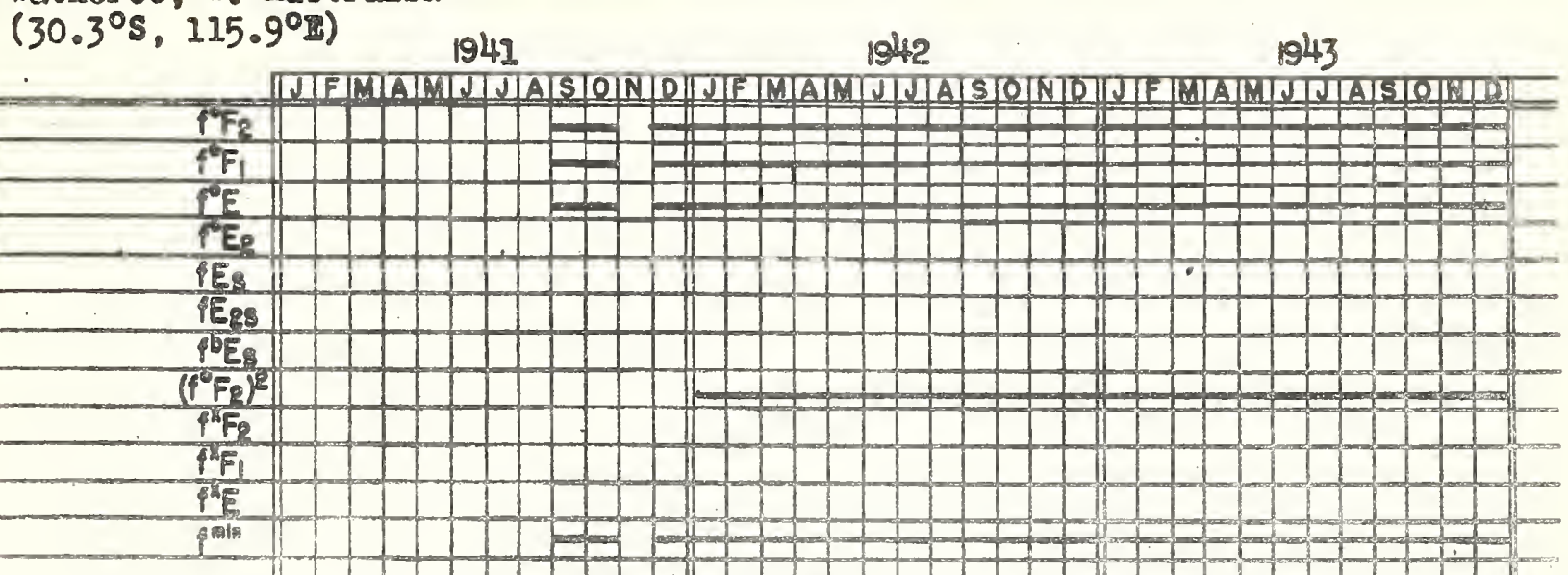

F23500mui

Fis000mat

(11) Fpl500nu?

End Felocoms?

(nd) Fe 500 mu

Find

5 ming

\begin{tabular}{r|}
\hline M500 \\
\hline$M 3000$ \\
\hline$M$ \\
\hline$M$ \\
\hline$M$ \\
\hline
\end{tabular}

\begin{tabular}{r}
$* F_{2} M 3000$ \\
$* F_{2} M 4000$ \\
\hline$h F_{2}$ \\
$h F_{1}$ \\
$h E$ \\
$h E_{2}$ \\
$h^{\prime} E_{9}$ \\
$h^{\prime} E_{2}$ \\
$h^{m} F_{2}$ \\
$h^{m} F_{1}$ \\
$h^{\text {m }} E^{2}$
\end{tabular}

be (npper)

hel (apper)

Absorption

* Calculated by LRP 

Watheroo, W. Australia

IONOSPHERIC DATA RECORO

$\left(30.3^{\circ} \mathrm{S}, 115.9^{\circ} \mathrm{E}\right)$
1939
1938

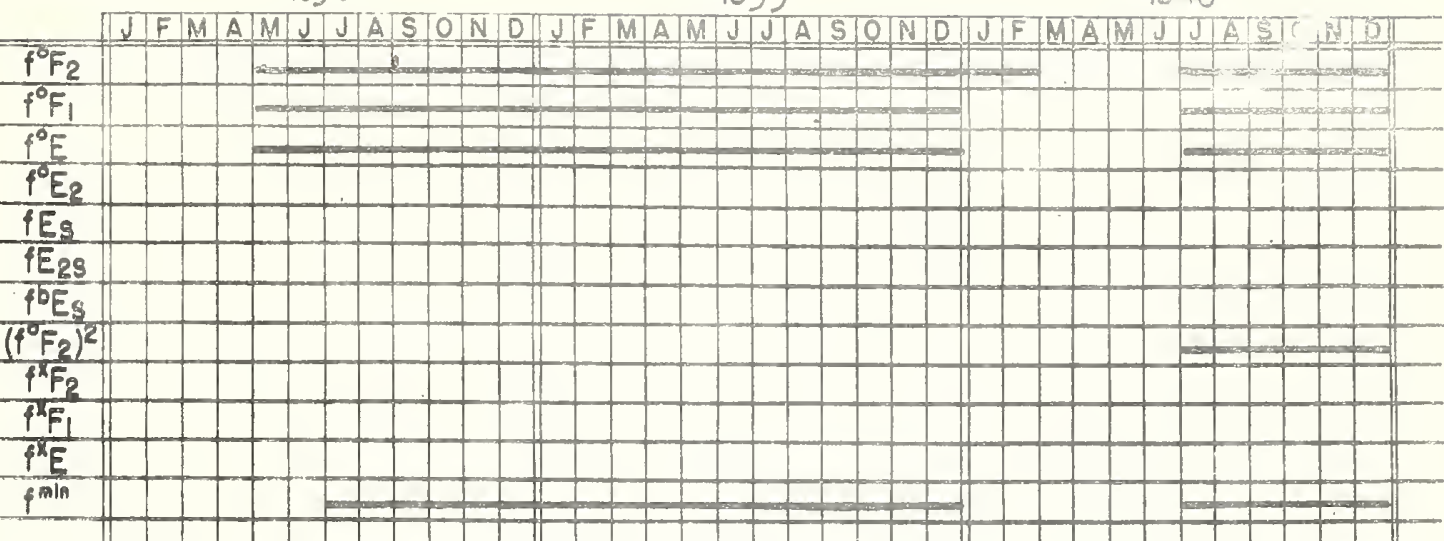

\begin{tabular}{rr|}
\hline$\frac{F}{F}$ & muf \\
\hline$\frac{F}{F}$ & muf \\
\hline$F$ & muf \\
\hline$F$ & muf \\
\hline$E$ & muf
\end{tabular}

$M 3500$

M3000

M

$M$

M

* $F_{2} M 3000$

* $F_{2} M 4000$

$\mathrm{h}_{2}$

$\mathrm{CF}_{1}$

h'E

$h^{\prime} E_{2}$

$h^{\prime} E_{s}$

$h^{\prime} E_{2 \mathrm{~s}}$

$h^{m} F_{2}$

$h^{m} E$

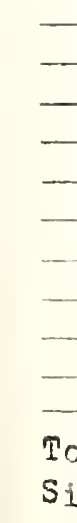


Watheroo, W. Australia IONOSPHERIC DATA RECORD

$\left(30.3^{\circ} \mathrm{S}, 115.90 \mathrm{~s}\right)$

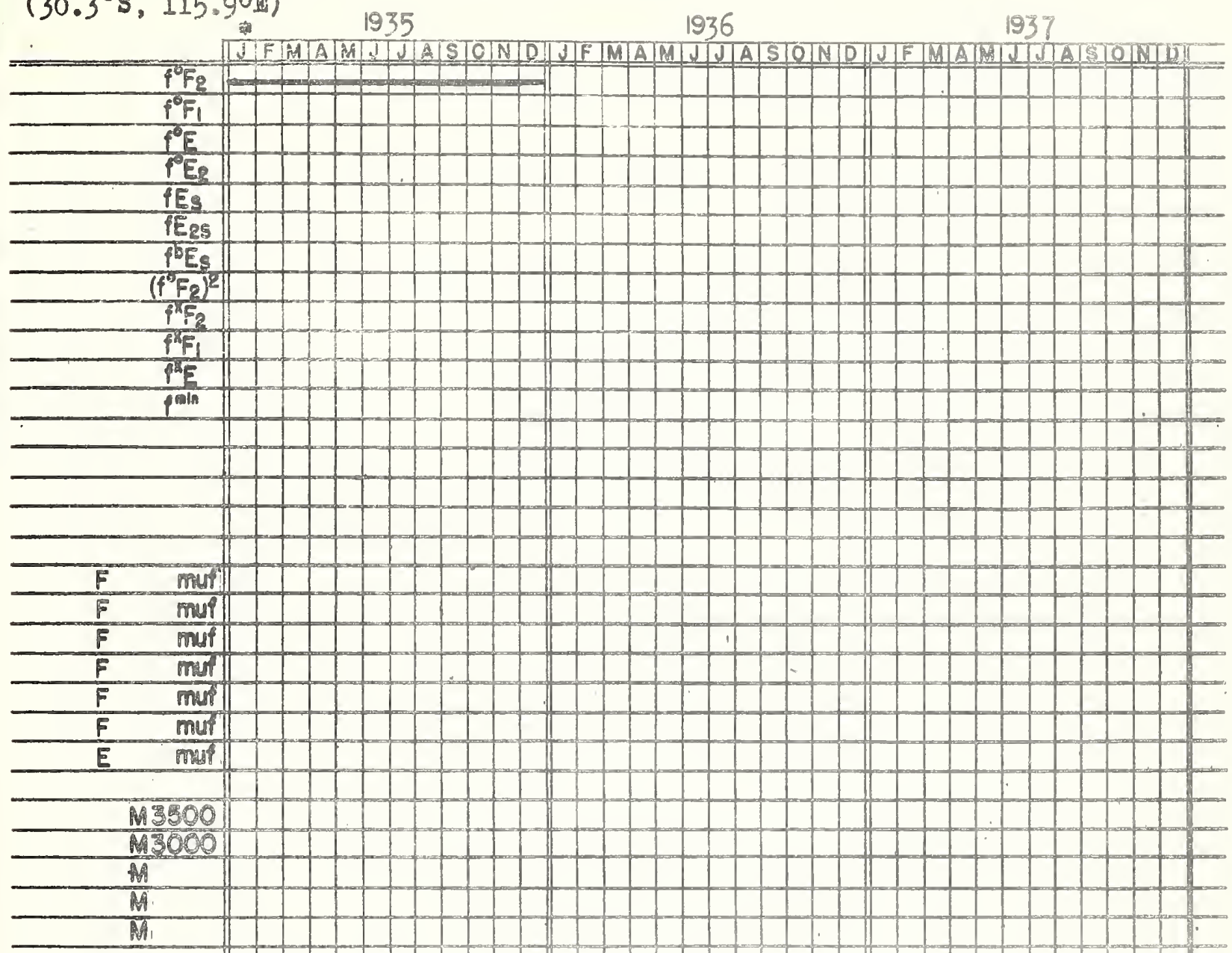

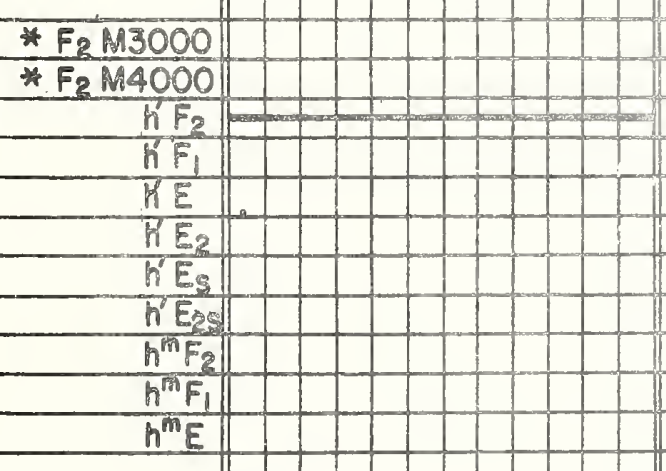

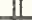



Wuchang, China

$(30.60 \%, 114.4 \%$

$$
1937 \text { 1938 }
$$

1939
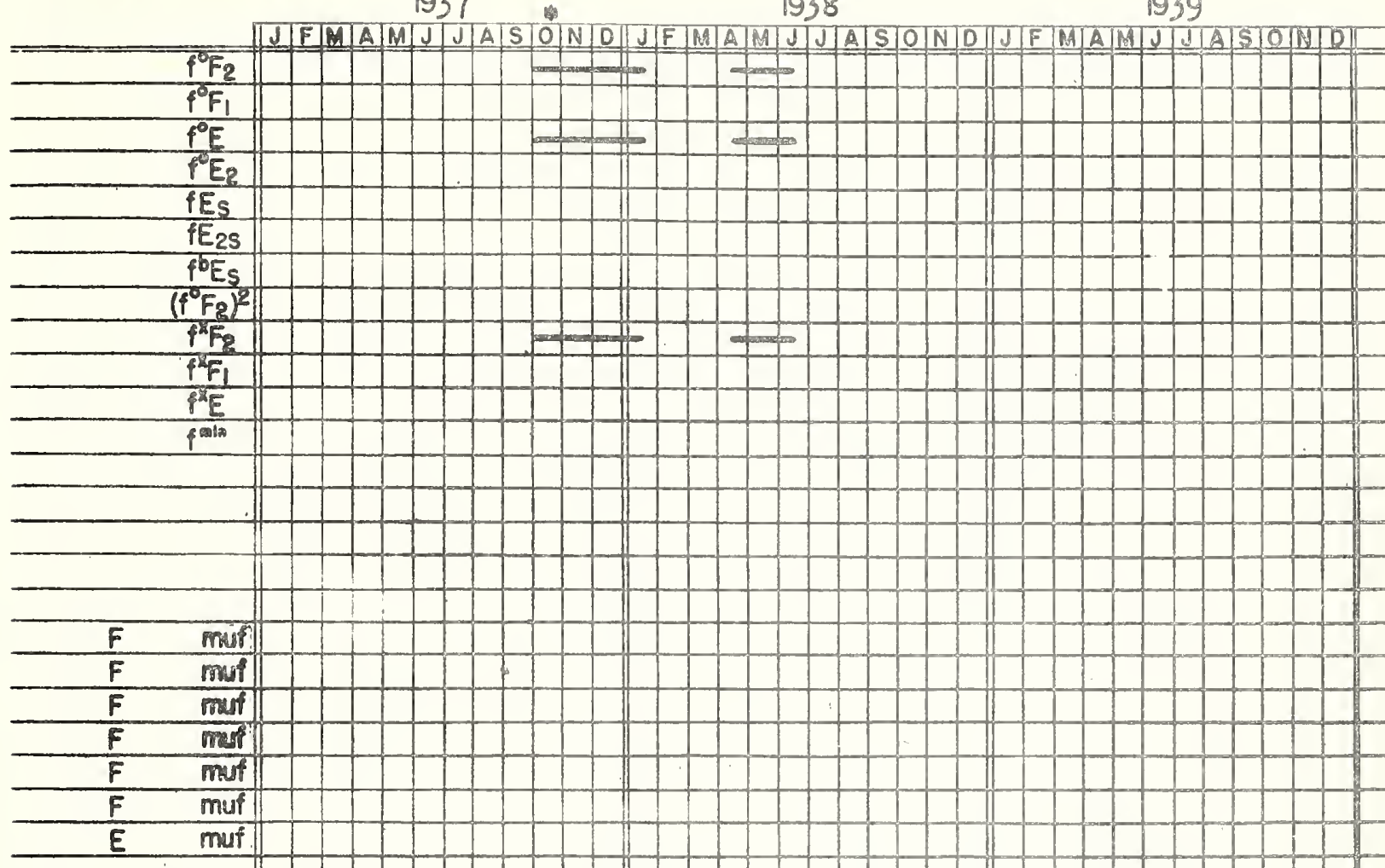

$\frac{M 3500}{M 3000}$

* $F_{2} M 3000$

* $F_{2} M 4000$

\begin{tabular}{l|}
$h^{\prime} F_{2}$ \\
$h^{\prime} F_{1}$ \\
$h^{\prime} E_{1}$ \\
$h^{\prime} E_{2}$ \\
$h^{\prime} E_{5}$ \\
$h^{\prime} E_{2}$ \\
$h^{m} F_{2}$ \\
$h^{m} F_{1}$ \\
$h^{m} E$
\end{tabular}

I

Absorption

* Calculated by IRPL

Oct. 4, 1937 through Jan. 23, 1938.

Apr. 25, 1938, through ins 1.6, 1938.

Woon and irrognlar evening runs. 



\begin{tabular}{ll|}
$F_{2}$ & muf \\
$F_{2}$ & muf \\
$F_{2}$ & muf \\
\hline$F_{2}$ & muf \\
\hline$F_{1}$ & muf \\
$F_{1}$ & muf \\
\hline$E$ & muf
\end{tabular}

$F_{2} M 3500$

$F_{2} M 3000$

$F_{2} M 1500$

$F_{1} M$

$F_{1} M$

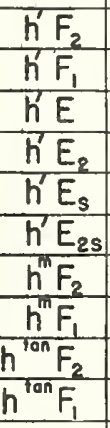




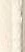


STATION IONOSPHERIC DATA RECORD

LAT. 19

LONG.

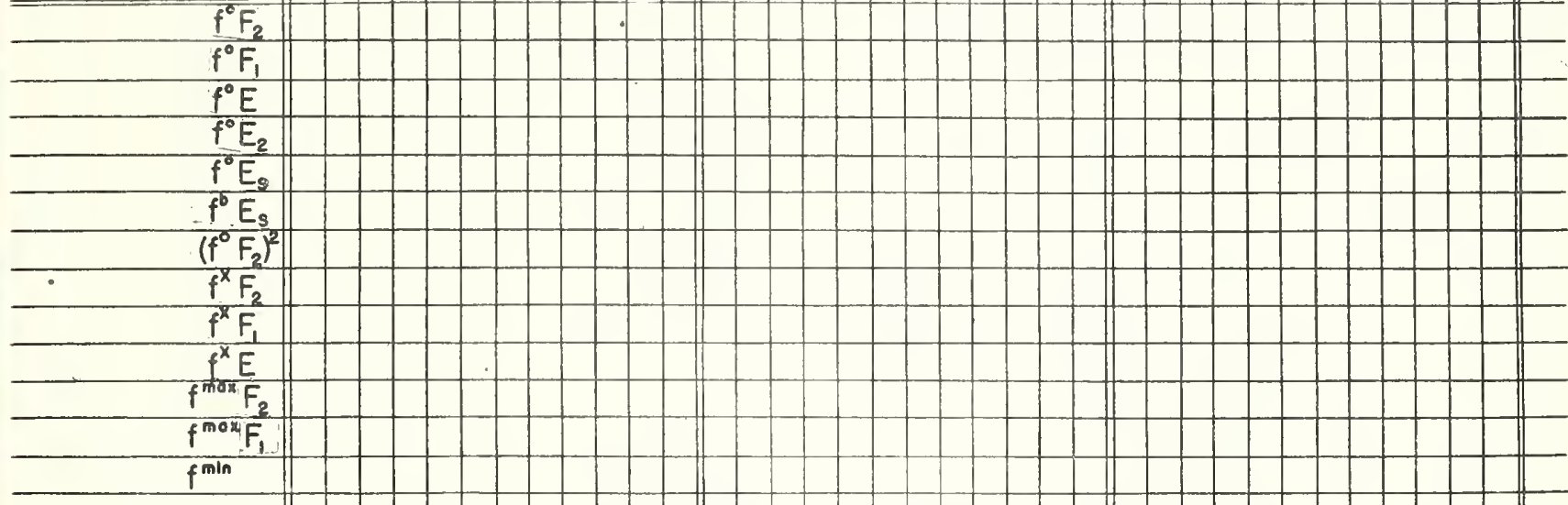

\begin{tabular}{|ll||}
$F_{2}$ & muf \\
\hline$F_{2}$ & muf \\
\hline$F_{2}$ & muf \\
$F_{2}$ & muf \\
$F_{1}$ & muf \\
$F_{1}$ & muf \\
\hline$E$ & muf
\end{tabular}

$\begin{array}{ll}F_{2} & M 3500 \\ F_{2} & M 3000 \\ F_{2} & M 1500 \\ F_{1} & M \\ F_{1} & M\end{array}$

\begin{tabular}{c|}
$h^{\prime} F_{2}$ \\
$h^{\prime} F_{1}$ \\
$h^{\prime} E^{\prime}$ \\
$h^{\prime} E_{2}$ \\
$h^{\prime} E_{s}$ \\
$h^{\prime} E_{2 s}$ \\
$h^{m} F_{2}$ \\
$h^{m} F_{1}$ \\
$\frac{h^{\text {ton }} F_{2}}{h^{\text {on }} F_{1}}$
\end{tabular} 


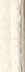


STATION

LAT.

LONG. IONOSPHERIC DATA RECORD

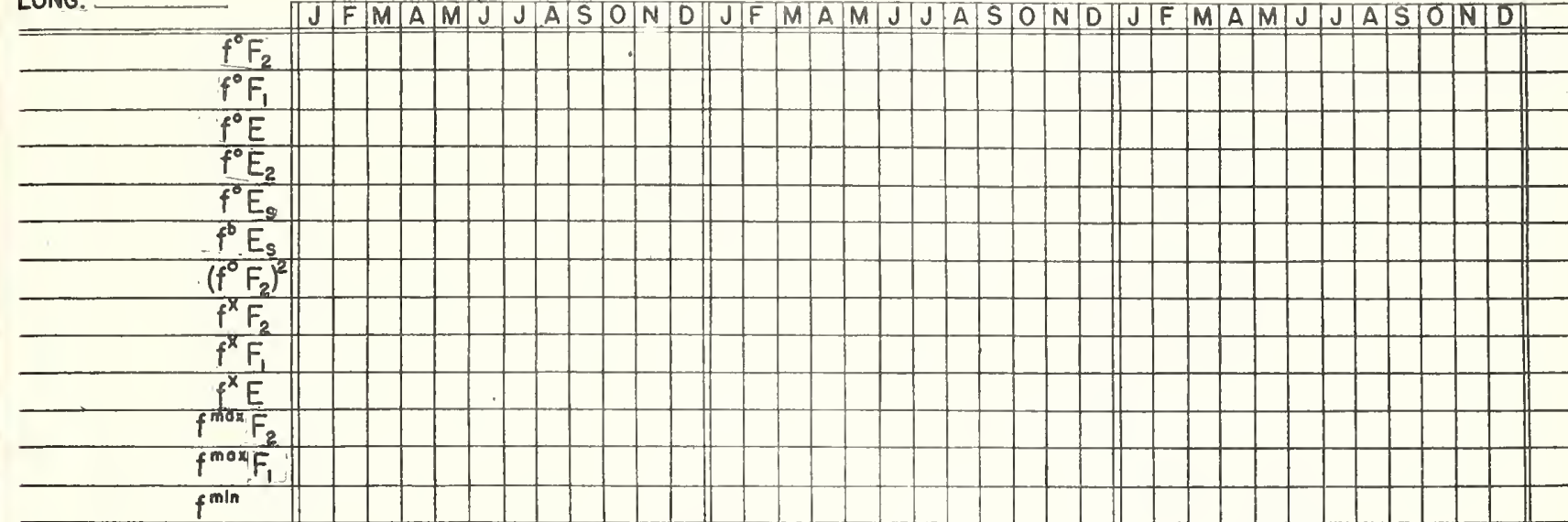

$F_{2} M 3500$
$F_{2} \cdot M 3000$
$F_{2} M 1500$
$F_{1} M$
$F_{1} \cdot M$

$h^{\prime} F_{2}$

$h^{\prime} F_{1}$

h'E

$h^{\prime} E_{2}$

$h^{\prime} E_{s}$

$h^{\prime} E_{2 s}$

$\mathrm{h}^{\mathrm{m}} \mathrm{F}_{2}$

$\frac{h^{\text {ran }} F_{2}}{h^{\text {lon }} F_{1}}$ 
STATION IONOSPHERIC DATA RECORD

LAT.

19

LONG. |J J F F M IA M M J J J A S S OO

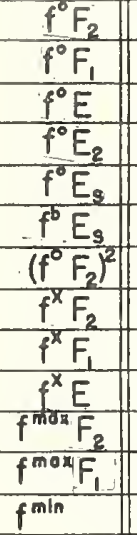

\begin{tabular}{ll}
$F_{2}$ & muf \\
$F_{2}$ & muf \\
$F_{2}$ & muf \\
$F_{2}$ & muf \\
$F_{1}$ & muf \\
$F_{1}$ & muf \\
\hline$E$ & muf
\end{tabular}

$F_{2} M 3500$

$F_{2} M 3000$

$F_{2} M 1500$

$F_{1} M$

$F_{1} M$

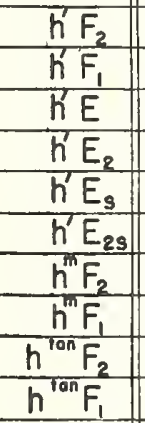



STATION IONOSPHERIC DATA RECORD

LAT. 19 19 19

LONG.

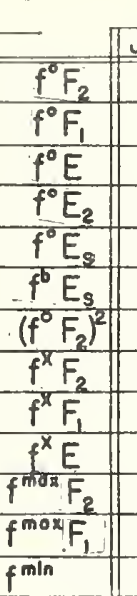

\begin{tabular}{ll}
$F_{2}$ & muf \\
\hline$F_{2}$ & muf \\
$F_{2}$ & muf \\
\hline$F_{2}$ & muf \\
$F_{1}$ & muf \\
$F_{1}$ & muf \\
\hline$E$ & muf
\end{tabular}

$F_{2} M 3500$

$F_{2} \cdot M 3000$

$F_{0} \quad M 1500$

$F_{1} M$

$F_{1} M$

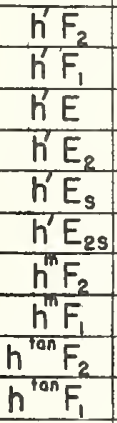

$\frac{I}{\text { Absorption }}$ 

STATION IONOSPHERIC DATA RECORD

LAT. 19

19

19

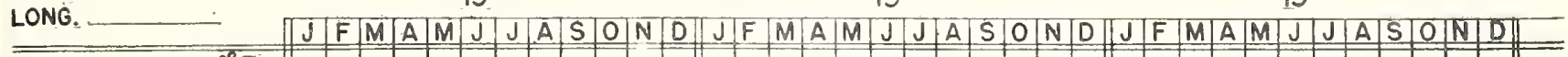

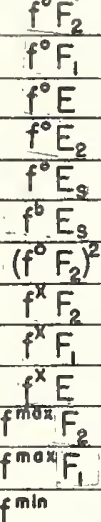

\begin{tabular}{|ll|}
\hline$F_{2}$ & muf \\
\hline$F_{2}$ & muf \\
\hline$F_{2}$ & muf \\
\hline$F_{2}$ & muf \\
\hline$F_{1}$ & muf \\
\hline$F_{1}$ & muf \\
\hline$E$ & muf
\end{tabular}

$F_{2} M 3500$

$F_{2} \cdot M 3000$

$F_{2} M 1500$

$F_{1} M$

$F_{1} M$

$h^{\prime} F_{2}$
$h^{\prime} F_{1}$
$h^{\prime} E$
$h^{\prime} E_{2}$
$h^{\prime} E_{s}$
$h^{\prime} E_{23}$
$h^{m} F_{2}$
$h^{m} F_{1}$
$h^{\text {ron }} F_{2}$
$h^{\text {ron }} F_{1}$

I

Absorption 

STATION

IONOSPHERIC DATA RECORD

LAT. 19

LONG.

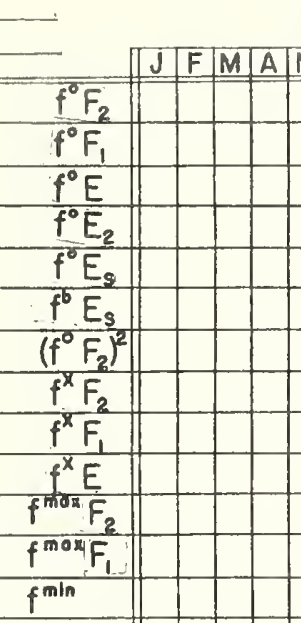

\begin{tabular}{ll}
$F_{2}$ & muf \\
\hline$F_{2}$ & muf \\
\hline$F_{2}$ & muf \\
\hline$F_{2}$ & muf \\
\hline$F_{1}$ & muf \\
\hline$F_{1}$ & muf \\
\hline$E$ & muf
\end{tabular}

$F_{2} M 3500$

$F_{2} M 3000$

$\mathrm{F}_{8} \mathrm{M} 1500$

$F_{1} M$

$F_{1} M$

$h^{\prime} F_{2}$
$h^{\prime} F_{1}$
$h^{\prime} E$
$h^{\prime} E_{2}$
$h^{\prime} E_{s}$
$h^{\prime} E_{2 s}$
$h^{\prime \prime \prime} F_{2}$
$h^{m} F_{1}$
$h^{100} F_{2}$
$h^{10 n} F_{1}$



STATION IONOSPHERIC DATA RECORD

LAT. 19

LONG.

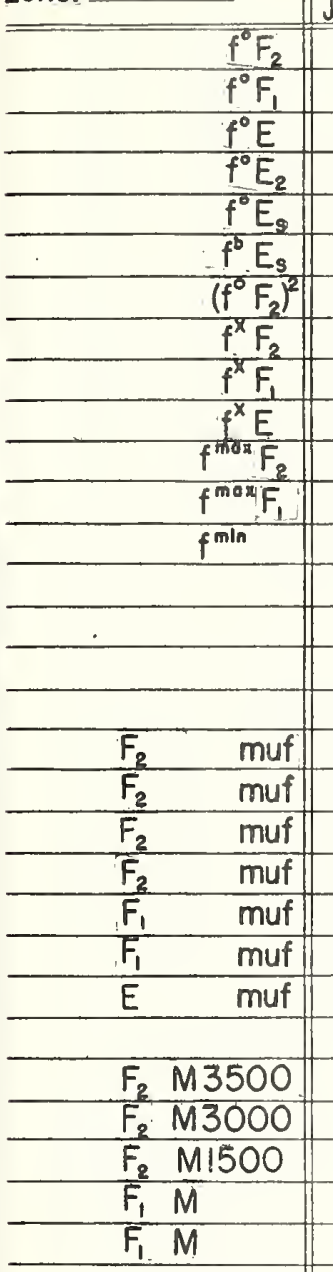

$h^{\prime} F_{2}$

$h^{\prime} F_{1}$

hE

$h^{\prime} E_{2}$

$h^{\prime} E_{s}$

$h^{\prime} E_{2 s}$

$h^{h \prime} F_{2}$

$h^{\prime \prime \prime} F_{1}$

$\frac{h^{\text {Tan }} F_{2}}{h^{\text {tan }} F_{1}}$ J FIM A M M J J J A S S O N N D 
